\title{
Generalized dynamical mean-field theory in physics of strongly correlated systems
}

\author{
E. Z. Kuchinskii ${ }^{1}$, I. A. Nekrasov ${ }^{1}$, M. V. Sadovskii ${ }^{1,2}$ \\ ${ }^{1}$ Institute for Electrophysics, Russian Academy of Sciences, Ural Branch, \\ Amundsen str. 106, Ekaterinburg 620016, Russia \\ ${ }^{2}$ Institute for Metal Physics, Russian Academy of Sciences, Ural Branch, \\ S. Kovalevskaya str. 18, Ekaterinburg 620219, Russia
}

\begin{abstract}
This review is devoted to generalization of dynamical mean-field theory (DMFT) for strongly correlated electronic systems towards the account of different types of additional interactions, necessary for correct physical description of many experimentally observed phenomena in such systems. As additional interactions we consider: (1) interaction of electrons with antiferromagnetic (or charge) fluctuations of order parameter in high-Tc superconductors leading to the formation of pseudogap state, (2) scattering of electrons on static disorder and its role in general picture of Anderson-Hubbard metal-insulator transition, (3) electron-phonon interaction and corresponding anomalies of electronic spectra in strongly correlated systems. Proposed DMFT $+\Sigma$ approach is based on taking into account above mentioned interactions by introducing additional self-energy $\Sigma$ (in general momentum dependent) into conventional DMFT scheme and calculated in a self-consistent way within the standard set of DMFT equations Here we formulate general scheme of calculation of both one-particle (spectral functions and densities of states) and two-particle (optical conductivity) properties. We examine the problem of pseudogap formation, including the Fermi arc formation and partial destruction of the Fermi surface, metal-insulator transition in disordered Anderson-Hubbard model, and general picture of kink formation within electronic spectra in strongly correlated systems.

$\mathrm{DMFT}+\Sigma$ approach is generalized to describe realistic materials with strong electron-electron correlations based on LDA+DMFT method. General scheme of LDA+DMFT method is presented together with some of its applications to real systems. The LDA $+\mathrm{DMFT}+\Sigma$ approach is employed to modelling of pseudogap state of electron and hole doped high- $\mathrm{T}_{c}$ cuprates. Comparison with variety of ARPES experiments is given.
\end{abstract}

PACS: 71.10.Fd, 71.10.Hf, 71.20.-b, 71.27.+a, 71.30.+h, 72.15.Rn, 74.72.-h 


\section{Contents}

1 Introduction 3

2 Strongly correlated systems and dynamical mean-field theory (DMFT). 4

2.1 Hubbard model and basics of DMFT. . . . . . . . . . . . . . . . . . . . . .

2.2 Generalized DMFT $+\Sigma$ approach. . . . . . . . . . . . . 7

2.3 Some other generalizations of DMFT . . . . . . . . . . . . . 10

3 Application of generalized DMFT $+\Sigma$ approach to model problems. 11

3.1 Strongly correlated systems in the pseudogap state. . . . . . . . . . . . 11

3.1.1 Pseudogap fluctuations. . . . . . . . . . . . . . . . . . 11

3.1.2 Basic electronic properties in the pseudogap state. . . . . . . . . . . 14

3.2 Mott-Anderson transition in disordered systems. . . . . . . . . . . . . . . . 19

3.2.1 Three-dimensional systems. . . . . . . . . . . . . . . . . 21

3.2.2 Two-dimensional systems. . . . . . . . . . . . . . . . 26

3.3 Singularities of electron dispersion in strongly correlated systems in DMFT

and DMFT $+\Sigma$ approaches. . . . . . . . . . . . . . . . 29

3.3.1 Cusps ("kinks") in electron spectra. . . . . . . . . . . . 29 29

3.3.2 Kinks of purely electronic nature . . . . . . . . . . . . . . . 30

3.3.3 Role of electron-phonon interaction . . . . . . . . . . . . . . 34

3.3.4 Electronic and phonon kinks within the DMFT $+\Sigma$ approach . . . 35

4 Electronic structure of real strongly correlated systems: LDA+DMFT and LDA+DMFT $+\Sigma \quad 37$

4.1 Density functional theory (DFT). Local density approximation (LDA). . . 37

4.2 LDA+DMFT computational scheme . . . . . . . . . . . . . . . . . . . . . . . . . . . . . .

4.3 Examples of LDA+DMFT calculations. . . . . . . . . . . . . . . . 41

4.3.1 Cubic perovskites $\mathrm{CaVO}_{3}$ and $\mathrm{SrVO}_{3} \ldots \ldots \ldots \ldots$. . . . . 41

4.3.2 Kinks in spectral function of $\mathrm{SrVO}_{3}$. . . . . . . . . . . . . 44

4.4 Electronic structure of copper oxides in the pseudogap state:

$\mathrm{LDA}+\mathrm{DMFT}+\mathrm{E} \ldots \ldots \ldots \ldots \ldots$

\begin{tabular}{ll|l}
5 & Conclusion & 52
\end{tabular}

6 Acknowledgements 53 


\section{Introduction}

Strongly correlated electronic systems (SCS), which are mainly realized in a number compounds of transition or rare-earth elements with partially filled $3 d, 4 f$ and $5 f$ shells, for more than half a century attract a lot of interest of scientists because of their unusual physical properties and difficulties in their theoretical description. Problem of metal-insulator phase transition, observed in many transition metal oxides, heavy fermions systems, with great variety of different phase transitions and related phenomena, manganites with giant magnetoresistance - all these systems become a subject of great attention for both experimentalists and theorists. Perhaps the most significant development in this area was the discovery of high temperature superconductivity in copper oxides, which provoked a new wave of interest in the synthesis and description of such systems.

As already stressed above, the diversity of physical phenomena in all these compounds is due to partially filled $3 d, 4 f$ and $5 f$ shells. Strong interaction of electrons within narrow bands belonging to these orbitals shells with each other or with itinerant electrons of outer shells is basically responsible for unique properties of these systems. Early qualitative ideas formulated by Mott [1] were further developed in many theoretical works to follow, forming a new area of modern theory of condensed matter. There are now thousands of such papers and many new reviews and books are regularly published [2].

Of course, nowadays, a single review can not cover all this area of research and the aim of authors is rather modest. The object of this work is description of a number of theoretical approaches formulated during recent years to account for some additional interactions which are important for the coherent description of strongly correlated systems. Here we speak not only about "external" perturbations due to interaction of correlated electrons with bosonic excitations such as phonons, spin fluctuations or scattering of electrons by disorder, but also about attempts to improve most developed and widely used theoretical approaches such as dynamical mean-field theory (DMFT) [3, 4, 5, 6].

These tasks are closely related with variety of topical problems under active study at present, such as the nature of the pseudogap state of high- $\mathrm{T}_{c}$ cuprates, the evolution of their Fermi surfaces upon doping with formation of so called "Fermi arcs", observed in ARPES experiments, the problem of formation of kinks in electronic spectrum, the general problem of metal-insulator transition in disordered systems, due to mutual interference of strong correlations and Anderson localization. In this review to some extent we deal with all of these problems.

During last years the general theory of strongly correlated systems based on DMFT practically merged with the so called "first-principle" approaches to calculations of electronic spectra of real solids (LDA+DMFT approach), with significant successes already

achieved [2, 7]. To this end in our review we devote some attention to first attempts of generalizing these approaches towards the account of the above mentioned physical effects. 


\section{Strongly correlated systems and dynamical mean- field theory (DMFT).}

\subsection{Hubbard model and basics of DMFT.}

Starting with pioneering works of Hubbard [8] in the early 60th the simplest model to describe strongly correlated systems is the so called Hubbard model. One band Hubbard model Hamiltonian is:

$$
H=-t \sum_{\langle i j\rangle \sigma} c_{i \sigma}^{\dagger} c_{j \sigma}+U \sum_{i} n_{i \uparrow} n_{i \downarrow}
$$

where $t>0$ - nearest neighbors hopping amplitude, $U$ - single site repulsion, $n_{i \sigma}=c_{i \sigma}^{\dagger} c_{i \sigma}-$ particle number operator on site $i, c_{i \sigma}\left(c_{i \sigma}^{\dagger}\right)$ - annihilation (creation) electron operators for spin $\sigma$. The model has only two competing energy parameters. Parameter $t$ defines kinetic energy of electron and facilitates intersite hoppings (delocalization), while parameter $U$ defines potential energy and favors localization of an electron on a lattice cite. Energy bands formed by $3 d, 4 f$ and $5 f$ orbitals are rather narrow, thus quite often kinetic and potential energy are of the same order of magnitude $(t \sim U)$. In this case there is no small parameter in the model and it is impossible to build any kind of perturbation theory. This fact alone leads to all the difficulties in description of SCS even for such oversimplified model.

Almost for 30 years there were no satisfactory approaches to analyze SCS. It seemed that theory of these systems will forever remain fragmentary and semiquantitative. The breakthrough came in 1989 in the work by Metzner and Vollhardt [9]. They suggested formal consideration of the system of interacting electrons in large space dimensions $d \rightarrow$ $\infty$ (or in a lattice with large coordination number $z \rightarrow \infty 1$ ).

Employing this limit it is possible to neglect spatial fluctuations in the systems while full local dynamics is preserved. In Ref. [9] it was shown that in the limit of infinite spatial dimensions (or more precise infinite coordination number) the main role is played only by local contributions to self-energy part of full interacting Green's function. All non local contributions are proportional to $1 / \sqrt{z} \sim 1 / \sqrt{d}$ and can be dropped. In this limit electron self-energy does not depend on momentum $\mathbf{k}$, and is a function of frequency only (real $(\omega)$ or Matsubara $\left(\omega_{n}\right)$ one) 2 :

$$
\Sigma_{\sigma}(\mathbf{k}, \omega)=\Sigma_{\sigma}(\omega)
$$

This statement is the main simplification appearing in the limit of $d \rightarrow \infty[3,5,6,6]$.

In Fig. 1 we show the "skeleton" diagrams of DMFT self-energy $\Sigma$. Wavy lines represent local (Hubbard) interaction $U$, full lines represent local Green functions $G_{i i}$. Strictly speaking in the limit of $d \rightarrow \infty$ the self-energy is not only a local one, but also in each vertex of "skeleton" diagram, only one particular site enters, e.g. the $i$-th ones, as shown in Fig. 1. Thus this self-energy is a functional $\Sigma_{i i, \sigma}=F\left[G_{i i, \sigma}\right]$ of interacting local Green's function $G_{i i, \sigma}$. Generally it is not sufficient to make our problem the fully

\footnotetext{
${ }^{1}$ For hypercubic lattice $z=2 d$ and these two limits practically coincide. However, even for three dimensional lattices $z$ could be quite large, for example, in body centered lattice $z=8$ and for face centered $z=12$. To this end it is more correct to speak about limit of large $z$.

${ }^{2}$ Large coordination numbers allow one to apply this approximation rather successfully even for rather small $d$
} 


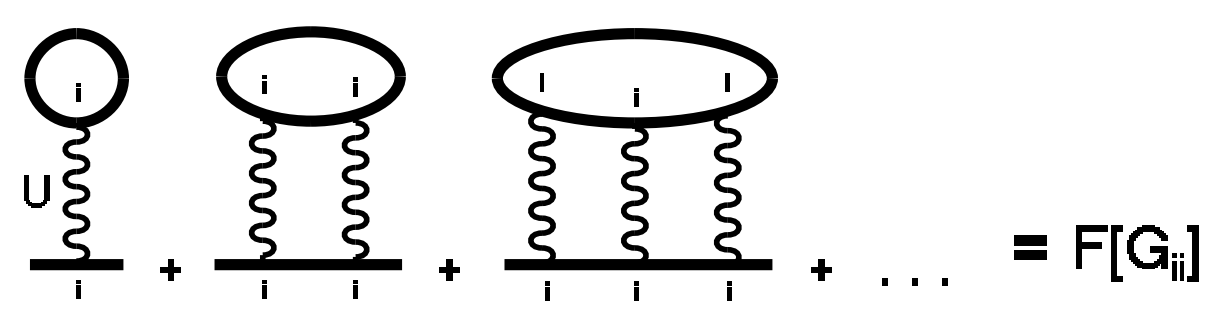

Figure 1: "Skeleton diagrams of local self-energy $\Sigma$ in DMFT.

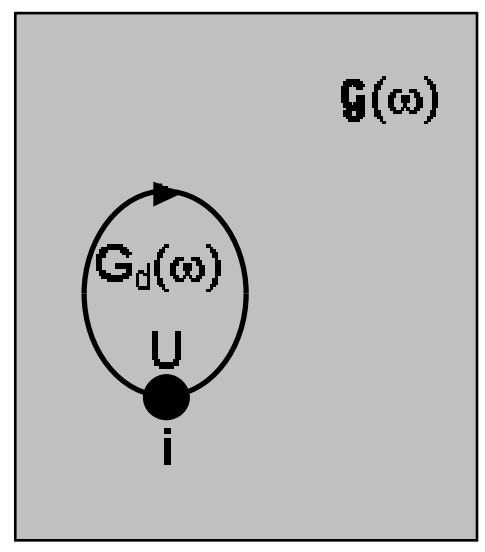

Figure 2: Within the DMFT lattice Hubbard model maps to interacting electrons on a single site ("impurity"), surrounded by the fermionic bath defining dynamical (Weiss) mean-field $\mathcal{G}(\omega)$.

local one, since interacting Green function $G_{i j, \sigma}$ is still nonlocal. Then a question arises, whether it is possible to choose purely local non interacting (in the absence of $U$ ) problem with completely equivalent self-energy? Surely it can be done! Let $\mathcal{G}_{\sigma}(\omega)$ to be "bare" dynamical Green function of such local problem without Coulomb interaction $U$, while $G_{d \sigma}(\omega)$ and $\Sigma_{d \sigma}(\omega)$ are corresponding interacting Green's function and self-energy. If one can guarantee the equality $G_{d \sigma}(\omega)=G_{i i, \sigma}(\omega)$, then corresponding self-energies are also equivalent, because the structure of diagrams of weak coupling $U$ perturbation theory is totally preserved, which means that self-energy of the local problem is defined by the same functional $\Sigma_{d \sigma}=F\left[G_{d \sigma}\right]$. But $\mathcal{G}_{\sigma}, G_{d \sigma}$ and $\Sigma_{d \sigma}$ are connected through the Dyson equation, which immediately gives us the "bare" dynamical Green function of the local problem.

$$
\mathcal{G}_{\sigma}^{-1}(\omega)=\Sigma_{\sigma}(\omega)+G_{i i, \sigma}^{-1}(\omega)
$$

Thus the lattice Hubbard model in the limit of $d \rightarrow \infty$ exactly maps onto purely local dynamical problem. Physically it corresponds (as shown in Fig. 2) to the problem of interacting electrons on a single "Anderson impurity" in a "bath" and interaction with the bath is contained in dynamical mean-field $\mathcal{G}(\omega)$. Quite often, in analogy with molecular field theory in magnetism, this field is called "Weiss field". This explains the name of such an approach - dynamical mean-field theory (DMFT).

This purely dynamic problem is still quite complicated. However, the problem is equivalent to a single impurity Anderson model (SIAM) [10]. This model can be studied in detail by a number of different methods and its physics is now well understood. For this model there are well developed approximate analytical methods such as iterative perturbation theory (IPT) [5] and non-crossing approximation (NCA) [11, 12], but most 


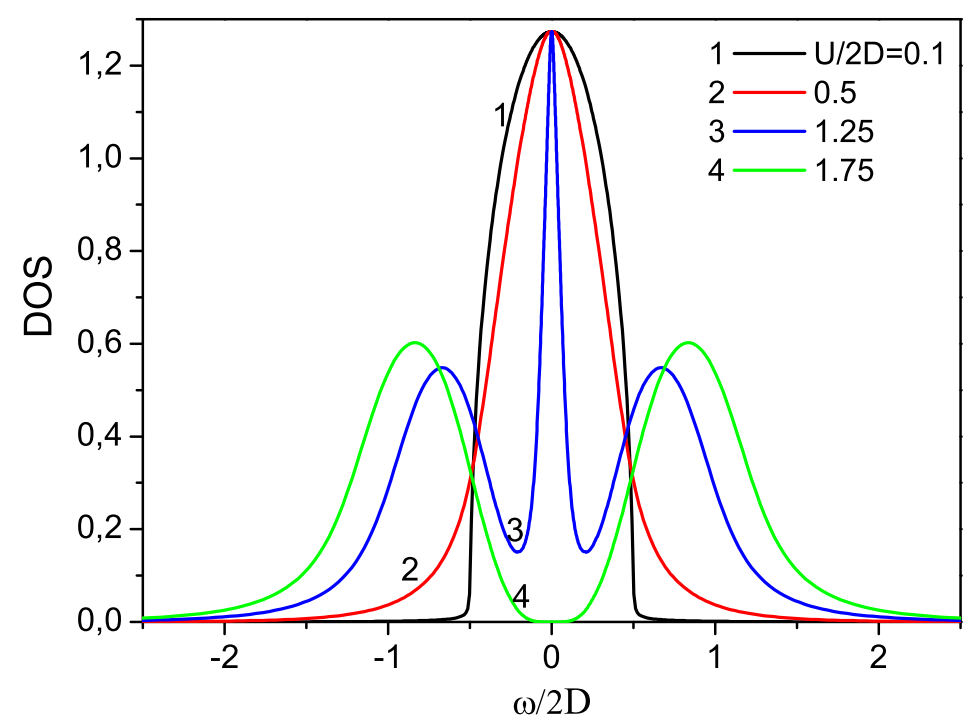

Figure 3: DMFT(NRG) densities of states at half-filling obtained for semielliptic "bare" density of states for different values of $U$.

remarkable is the possibility to solve this model by exact numerical methods like quantum Monte-Carlo (QMC) [13] or numerical renormalization group (NRG) [14, 15]. Solution of an effective SIAM employing any of these methods, usually called an "impurity solver", completes the general scheme of DMFT approach.

Apparently, today DMFT is the most elaborate and reliable theoretical method to describe SCS. In its framework the so called three-peak structure of the density of states of SCS was obtained for the first time [5], consisting of the central (quasiparticle) peak on the Fermi level and two wide maxima, corresponding to upper and lower Hubbard bands. Also the reliable theoretical description of Mott-Hubbard metal-insulator transition was obtained. In Fig. 3 we show DMFT(NRG) densities of states of the half-filled Hubbard model with semielliptic "bare" density of states with bandwidth $2 D$. As correlation strength $U$ grows the density of states demonstrates the formation of characteristic threepeak structure and further increase of $U$ leads to a collapse of the quasiparticle peak at $U / 2 D \approx 1.5$, leading to metal-insulator transition.

It turns out that within DMFT it is also possible to investigate some two-particle properties. In particular, it is quite easy to obtain dynamic optical conductivity [5, 4]. During the recent years DMFT approach was generalized to describe realistic SCS merging it with "ab initio" one-electron density functional theory in local density approximation (DFT/LDA), leading to the combined computational scheme of LDA+DMFT [16, 17, 18, 19, 20, which will be described later.

Despite all the obvious advantages of DMFT this approach has a number of shortcomings. Namely, as we stressed above, all non-local correlations are completely neglected. A number of cluster generalizations of DMFT [21, 22] were proposed recently to overcome this drawback. However, all these methods are quite computer time consuming and are rather restricted in a cluster size and with respect to their generalization to multi-orbital case. Also in these approaches it is quite difficult to investigate two-particle properties. To overcome these difficulties we proposed recently [23, 24, 25] the new generalization of the conventional DMFT, allowing to consider non-local correlations or additional (with respect to the Hubbard one) interactions (in principle of any kind), remaining within a 
single impurity DFMT picture and preserving self-consistent set of DMFT equations.

\subsection{Generalized DMFT $+\Sigma$ approach.}

The main idea of the new approach is to use the exact in the limit of $d \rightarrow \infty$ DMFT solution as a "high energy" zeroth order approximation, describing electronic spectra on a large energy scale of the order of bandwidth or $U$ value, while low energy scale details caused by non-local effects or by effects of interaction of correlated electrons with different collective modes (e.g. phonons or spin fluctuations) are to be taken into account within some kind of perturbation theory, conserving, as far as possible, the general structure of DMFT equations. Actually, such a scheme can be realized in rather simple way [23, 24, 25].

To be more specific, in the following we consider the standard one band Hubbard model. Generalizations towards multi orbital case are also possible. Main assumption of our approach is to choose Matsubara lattice Green's function as:

$$
G_{\mathbf{k}}(i \omega)=\frac{1}{i \omega+\mu-\varepsilon(\mathbf{k})-\Sigma(i \omega)-\Sigma_{\mathbf{k}}(i \omega)}, \quad \omega=\pi T(2 n+1),
$$

where $\mu$ is the chemical potential, $\Sigma(i \omega)$ - local DMFT self-energy due to Hubbard interaction and $\Sigma_{\mathbf{k}}(i \omega)$ - some "external" (generally non-local, momentum dependent) selfenergy. This last contribution can arise from interaction of correlated electrons with some "additional" collective modes or order parameter fluctuations appearing in the Hubbard model itself, or from any other interactions (fluctuations) external with respect to the standard Hubbard model. For example these can be phonons or scattering by impurities, when it is actually local (momentum independent).

One should emphasize that $\Sigma_{\mathbf{k}}(i \omega)$ can contain local (momentum independent) contribution even if the self-energy is considered in the framework of the Hubbard model. However this contribution disappears in the infinite spatial dimensions limit of $d \rightarrow \infty$ and is not accounted within the conventional DMFT, so that within our approach we not encounter any double counting problem even in this case. This question does not come out at all for self-energy $\Sigma_{\mathbf{k}}(i \omega)$ caused by "external" interactions.

More important is that our assumption of the additive form of a self-energy $\Sigma(i \omega)+$ $\Sigma_{\mathbf{k}}(i \omega)$ implicitly corresponds to the neglect of interference of the local (DMFT) and nonlocal contributions. In Fig. 4 typical "skeleton" diagrams for self-energy of DMFT $+\Sigma$ approach are given. First two terms are local DFMT self-energy diagrams, two diagrams in the middle show contributions to non-local part of self-energy from "additional" interactions with collective modes or order parameter fluctuations, and the last diagram (b) is an example of diagram with interference between local and non-local parts which is neglected. Indeed, once we neglect such interference (i.e. diagram shown in Fig. 4(b)) the total self-energy is defined as a simple sum of these two contributions shown in Fig. 4. Two last diagrams in Fig. 4(a) are an example of "skeleton" diagrams for non-local self-energy, where full line is the Green's function $G_{\mathbf{k}}$ (4) and dashed line corresponds to an "additional" interaction with collective modes or order parameter fluctuations.

Finally, diagrammatic structure of the local self-energy remains identical to that of the standard DMFT and we obtain the following self-consistent equations of the generalized DMFT $+\Sigma$ approach [23, 24, 25]:

1. Start from some initial guess for the local self-energy $\Sigma(i \omega)$, e.g., $\Sigma(i \omega)=0$. 


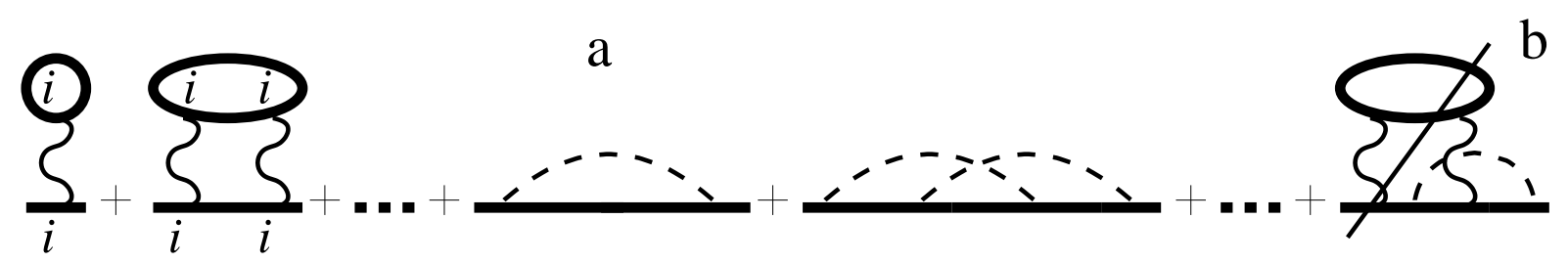

Figure 4: Typical "skeleton" self-energy diagrams of DMFT $+\Sigma$ approach.

2. Calculate self-energy $\Sigma_{\mathbf{k}}(i \omega)$ in the framework of some (approximate) scheme, taking into account interaction of correlated electrons with collective modes or order parameter fluctuations, which, in general, can depend on $\Sigma(i \omega)$ and $\mu$.

3. Calculates local Green's function:

$$
G_{i i}(i \omega)=\frac{1}{N} \sum_{\mathbf{k}} \frac{1}{i \omega+\mu-\varepsilon(\mathbf{k})-\Sigma(i \omega)-\Sigma_{\mathbf{k}}(i \omega)} .
$$

4. Define the "Weiss field" as:

$$
\mathcal{G}_{0}^{-1}(i \omega)=\Sigma(i \omega)+G_{i i}^{-1}(i \omega)
$$

5. Using some "impurity solver" calculate the single-particle Green's function of an effective single impurity Anderson model, i.e. compute the following integral over Grassmann variables $c_{i \sigma}^{+}$and $c_{i \sigma}$ :

$$
G_{d}\left(\tau-\tau^{\prime}\right)=\frac{1}{Z_{\mathrm{eff}}} \int D c_{i \sigma}^{+} D c_{i \sigma} c_{i \sigma}(\tau) c_{i \sigma}^{+}\left(\tau^{\prime}\right) \exp \left(-S_{\mathrm{eff}}\right)
$$

where an effective action for the fixed site ("Anderson impurity") $i$ is:

$$
S_{\mathrm{eff}}=-\int_{0}^{\beta} d \tau_{1} \int_{0}^{\beta} d \tau_{2} c_{i \sigma}\left(\tau_{1}\right) \mathcal{G}_{0}^{-1}\left(\tau_{1}-\tau_{2}\right) c_{i \sigma}^{+}\left(\tau_{2}\right)+\int_{0}^{\beta} d \tau U n_{i \uparrow}(\tau) n_{i \downarrow}(\tau)
$$

with "partition function" $Z_{\mathrm{eff}}=\int D c_{i \sigma}^{+} D c_{i \sigma} \exp \left(-S_{\mathrm{eff}}\right)$, and $\beta=T^{-1}$.

6. Define new local self-energy as:

$$
\Sigma(i \omega)=\mathcal{G}_{0}^{-1}(i \omega)-G_{d}^{-1}(i \omega)
$$

7. Using this self-energy as an "initial" on step 1, continue the loop procedure until it converges to

$$
G_{i i}(i \omega)=G_{d}(i \omega)
$$

with a given accuracy.

At the end we obtain the final Green's function in the form of Eq. (44), where $\Sigma(i \omega)$ and $\Sigma_{\mathbf{k}}(i \omega)$ are self-energies coming out of our iterative procedure.

Success of such approach (as well as its main drawback) is connected with an additive form of total self-energy (neglect of interference between different contributions) in Eq. (4). This allows one to preserve self-consistent set of equations of the standard DMFT. 
However there are two significant distinctions from conventional DMFT. First of all, the local Green's function of an effective single impurity problem has the form of Eq. (5) on each step of DMFT procedure. Secondly, during each DMFT iteration "external" self-energy $\Sigma_{\mathbf{k}}(i \omega)$ is recalculated within some (approximate) scheme, taking into account interaction with collective modes (phonons, magnons etc.) or with fluctuations of some order parameter. To define non-local contribution $\Sigma_{\mathbf{k}}(i \omega)$ it is convenient to introduce

$$
\mathcal{G}_{0 \mathbf{k}}(i \omega)=\frac{1}{G_{\mathbf{k}}^{-1}(i \omega)+\Sigma_{\mathbf{k}}(i \omega)}=\frac{1}{i \omega+\mu-\varepsilon(\mathbf{k})-\Sigma(i \omega)},
$$

which plays the role of "bare" Green's function to build perturbation theory over "external" interaction. The choice of the "bare" Green's function in the form of Eq. (11) guarantees Green's function "dressed" by such interaction $G_{\mathbf{k}}^{-1}(i \omega)=\mathcal{G}_{0 \mathbf{k}}^{-1}(i \omega)-\Sigma_{\mathbf{k}}(i \omega)$, entering "skeleton" diagrams for $\Sigma_{\mathbf{k}}(i \omega)$, coincides exactly with full Green's function $G_{\mathbf{k}}(i \omega)$.

Remarkable feature of our approach is the possibility of its generalization to calculate two-particle properties e.g. optical conductivity [26, 27]. Conductivity of a system is expressed via retarded density-density response function $\chi^{R}(\omega, \mathbf{q})[28,29]$ :

$$
\sigma(\omega)=-\lim _{q \rightarrow 0} \frac{i e^{2} \omega}{q^{2}} \chi^{R}(\omega, \mathbf{q}),
$$

where $e$ is electron charge. This response function is defined by analytical continuation to real frequencies of the full polarization loop in Matsubara representation [28]. Note that conductivity is completely defined by first derivative of this response function with respect to $q^{2}$ in the limit of $q \rightarrow 0$. This circumstance, as well as the neglect of interference between Hubbard and "external" interactions in DMFT $+\Sigma$ approach and locality of irreducible vertices of Hubbard interaction allows one to perform a partial resummation of diagrams relevant for conductivity, making the use of an exact (in the limit of $q \rightarrow 0$ ) Ward identity. At the end the real part of optical conductivity in the DMFT $+\Sigma$ approach is [26, 27]:

$$
\begin{aligned}
\operatorname{Re} \sigma(\omega)=\frac{e^{2} \omega}{2 \pi} \int_{-\infty}^{\infty} d \varepsilon\left[f\left(\varepsilon_{-}\right)-f\left(\varepsilon_{+}\right)\right] \operatorname{Re} & \left\{\phi_{\varepsilon}^{0 R A}(\omega)\left[1-\frac{\Sigma^{R}\left(\varepsilon_{+}\right)-\Sigma^{A}\left(\varepsilon_{-}\right)}{\omega}\right]^{2}-\right. \\
& \left.-\phi_{\varepsilon}^{0 R R}(\omega)\left[1-\frac{\Sigma^{R}\left(\varepsilon_{+}\right)-\Sigma^{R}\left(\varepsilon_{-}\right)}{\omega}\right]^{2}\right\} .
\end{aligned}
$$

where

$$
\phi_{\varepsilon}^{0 R R(R A)}(\omega)=\lim _{q \rightarrow 0} \frac{\Phi_{\varepsilon}^{0 R R(R A)}(\omega, \mathbf{q})-\Phi_{\varepsilon}^{0 R R(R A)}(\omega, 0)}{q^{2}},
$$

and we introduced the two-particle Green functions of the following form:

$$
\Phi_{\varepsilon}^{0 R R(R A)}(\omega, \mathbf{q})=\sum_{\mathbf{k}} G^{R}\left(\varepsilon_{+}, \mathbf{k}_{+}\right) G^{R(A)}\left(\varepsilon_{-}, \mathbf{k}_{-}\right) \Gamma^{R R(R A)}\left(\varepsilon_{-}, \mathbf{k}_{-} ; \varepsilon_{+}, \mathbf{k}_{+}\right)
$$

which are diagrammatically represented by Fig. $5\left(\mathbf{k}_{ \pm}=\mathbf{k} \pm \frac{\mathbf{q}}{2}, \varepsilon_{ \pm}=\varepsilon \pm \frac{\omega}{2}\right)$. Vertices $\Gamma^{R R(R A)}\left(\varepsilon_{-}, \mathbf{k}_{-} ; \varepsilon_{+}, \mathbf{k}_{+}\right)$contain all vertex corrections from "external" interaction (order parameter fluctuations, impurities, phonons etc.) but do not contain vertex corrections from Hubbard interaction. 


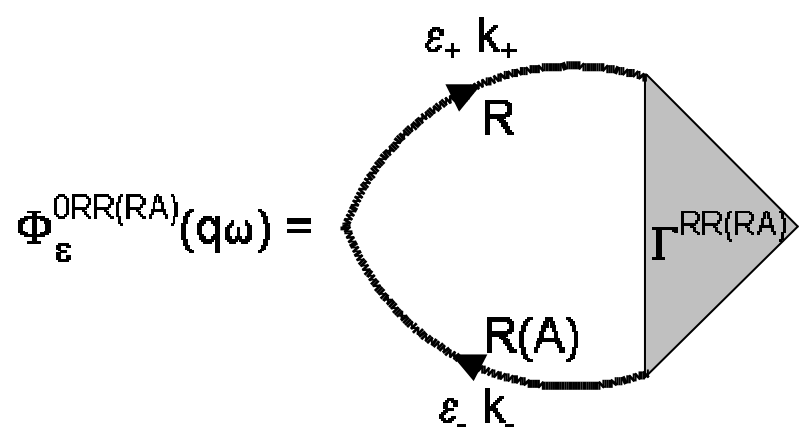

Figure 5: Diagrammatic representation of $\Phi_{\varepsilon}^{0 R A}(\omega, \mathbf{q})$ and $\Phi_{\varepsilon}^{0 R R}(\omega, \mathbf{q})$.

Thus one achieves a significant simplification of the problem. To calculate optical conductivity within DMFT $+\Sigma$ approach we have only to solve single-particle problem of obtaining the local self-energy $\Sigma\left(\varepsilon_{ \pm}\right)$with the help of DMFT $+\Sigma$ procedure described above, while non-trivial contribution from non-local correlations or "external" perturbations enters via "blocks" (14), which can be calculated in any suitable approximation accounting only for "external" interaction, with "bare" Green's functions (11), which contains the local self-energy from DMFT $+\Sigma$ procedure. In fact Eq. (13) provides also an effective algorithm to compute optical conductivity in framework of conventional DMFT (neglecting all "external" non-local correlations). In this case (14) could be easily found from the simple loop diagram, defined by two Green's functions and free scalar vertices. To get optical conductivity there is actually no need to calculate vertex corrections in the framework of DMFT itself, as it was first shown for the loop with vector vertices in Refs. [5, 4].

In the following, in Sections 3 and 4 we shall discuss in details some applications of the generalized DMFT $+\Sigma$ approach to the solution of concrete physical problems.

\subsection{Some other generalizations of DMFT}

Up to now, a number of different theoretical approaches were suggested to account for non-local effects within generalizations of DMFT. Here we restrict ourselves to a brief review of some of these approaches.

First of all we shall refer to cluster methods already mentioned above. Instead of an isolated Anderson impurity one can consider some cluster containing several such impurities, treating single site correlations within DMFT, while considering intersite correlations by some other method. This is basic idea of the so called cluster DMFT methods [5, 30, 31].

A drawback of such methods is related to different treatment of non-local correlation inside cluster and between clusters, though from the physical point of view (for example because of translational invariance) they should be identical. To overcome this difficulty it was proposed to average self-consistent "cluster" self-energy over pairs of sites connected by translation vectors [30]. Unfortunately such averaging procedure does not work well within the sel-consistent cycle of DMFT, since it breaks down analytical properties of Green's function.

In some sense alternative approach, named dynamical cluster approximation (DCA), was proposed in Refs. [31] and [32, 33]. This approach conserves translational invariance and provides physical behavior of Green's function. Within DCA the Brillouin zone is 
divided into several cells with centers defined by appropriate vectors $\mathbf{K}$. The same time self-energy is assumed to be constant in each cell $\Sigma_{\mathbf{k}}(\omega)=\Sigma_{\mathbf{K}}(\omega)$, but these constant are different for different $K$. Distinction between DCA and cluster DMFT is that in DCA DMFT-cluster satisfies periodic boundary conditions, instead of open boundary conditions in cluster DMFT.

The choice of particular cluster method is dictated by physical problem under consideration. Naturally, the common difficulty of cluster approaches is essentially larger computer time consumption in contrast to the standard DMFT, which is connected with the solution of the appropriate cluster problem. Nevertheless, a number of successes were achieved on this way. Cluster DMFT generalizations were applied to different models as well as to studies of some realistic systems. Detailed review of these works can be found in Ref. [21.

Recently a number of diagrammatic DMFT generalizations was proposed, attempting for more or less consistent construction of perturbation theory over the inverse powers of spatial dimensionality, with the standard DMFT used as the zeroth order approximation. We mention in this connection the Ref. [34] and also the so called dynamical vertex approximation (DГA), developed in Ref. [35]. Most promising in this respect seems to the dual fermion approach formulated in Refs. [36, 37], which is claimed to be a consistent realization of such perturbation theory. Unfortunately up to now only some simple model problems were solved by these methods, while realistic systems where not yet considered at all.

\section{Application of generalized DMFT $+\Sigma$ approach to model problems.}

\subsection{Strongly correlated systems in the pseudogap state.}

\subsubsection{Pseudogap fluctuations.}

Striking example of strongly correlated systems are high- $\mathrm{T}_{c}$ cuprates. Parent stoichiometric cuprate compounds are antiferromagnetic insulators with well developed optical gap and antiferromagnetism due to spin ordering on copper ions with Neel temperature of the order of hundreds of $K$. This insulating state is rapidly destroyed by introduction of rather few doping impurities. Thus these systems can be classified as doped Mott insulators with strong electronic correlations.

Among many anomalies of the normal phase of high temperature superconductors special interest attracts observations of a pseudogap in the electronic spectra of underdoped cuprates [38, 39]. Despite continuing discussions about pseudogap nature, from our point of view, most preferable is the scenario of pseudogap formation due strong scattering of charge carriers on antiferromagnetic (AFM, SDW) short range order fluctuations [39, 40]. In momentum representation this scattering is characterized by momentum transfer of the order of $\mathbf{Q}=\left(\frac{\pi}{a}, \frac{\pi}{a}\right)$ ( $a$ is two-dimensional lattice constant). This leads to formation of certain features in single-particle spectrum, which are precursors of changes in the spectra due to the appearance of AFM long range order (period doubling). As a result we end up with non Fermi liquid behavior (dielectrization) of spectral density in the vicinity of the so called "hot-spots" on the Fermi surface, appearing at intersections of Fermi surface 
with borders of AFM Brillouin zone [39].

In the framework of this spin-fluctuation scenario in works a simplified model of the pseudogap state was studied in Refs. [39, 41, 42. This model is based on the assumption that for high enough temperatures dynamics of spin fluctuations can be neglected and one can consider instead the scattering of charge carriers by static Gaussian random field (quenched disorder) of pseudogap fluctuations (short range order AFM fluctuations). Scattering intensity on fluctuations is characterized by a narrow peak near scattering vectors of the order of $\mathbf{Q}$ with a width defined by inverse correlation length $\kappa=\xi^{-1}$ and corresponding energy scale $\Delta$ (of the order of pseudogap crossover temperature $T^{*}$ ).

Thus for momentum dependent self-energy we shall concentrate on the case of electron scattering on such (SDW-like) antiferromagnetic spin fluctuations (similar consideration works fine also for CDW-like charge fluctuations) with short range order. To calculate $\Sigma_{\mathbf{k}}(i \omega)$ for the case of electrons propagating in quenched random field of Gaussian spin (or charge) fluctuations with dominating scattering momentum close to characteristic vector Q ("hot-spot" model [39]), we shall use the generalized version of recurrent procedure proposed in Refs. [41, 42, 43, allowing to take into account all Feynman diagrams describing scattering of electrons by this random field. This becomes possible because of remarkable property of simplified "hot-spot" model: contribution of arbitrary diagram with crossing interaction lines is equal to contribution of some diagram of the same order without crossing of those lines [43]. Thereby we can restrict ourselves to consideration of non-crossing diagrams only and take into account other diagrams by combinatorial prefactors attributed to interaction lines [42, 43]. Finally we obtain the following recurrent relation for the self-energy (continuous fraction representation [42, 43]):

$$
\Sigma_{n}(i \omega \mathbf{k})=\Delta^{2} \frac{s(n)}{i \omega+\mu-\Sigma(i \omega)-\varepsilon_{n}(\mathbf{k})+i n v_{n} \kappa-\Sigma_{n+1}(i \omega, \mathbf{k})} .
$$

Here, the term $\Sigma_{n}(i \omega, \mathbf{k})$ of recurrent procedure contains all diagrammatic contributions with number of interaction lines $\geq n$. Recurrent procedure for $\Sigma_{n}(i \omega, \mathbf{k})$ converges rather fast, we can put $\Sigma_{n}(i \omega, \mathbf{k})$ for large enough $n$ equal to zero and performing straightforward computations obtain the desired physical self-energy for $n=1$ [42], which can be subsequently used in DMFT $+\Sigma$ computational scheme:

$$
\Sigma_{\mathbf{k}}(i \omega)=\Sigma_{n=1}(i \omega, \mathbf{k})
$$

Parameter $\Delta$ characterizes the energy scale (width) of the pseudogap, $\kappa=\xi^{-1}$ is the inverse correlation length of SDW (CDW) fluctuations, $\varepsilon_{n}(\mathbf{k})=\varepsilon(\mathbf{k}+\mathbf{Q})$ and $v_{n}=$ $\left|v_{\mathbf{k}+\mathbf{Q}}^{x}\right|+\left|v_{\mathbf{k}+\mathbf{Q}}^{y}\right|$ for odd $n, \varepsilon_{n}(\mathbf{k})=\varepsilon(\mathbf{k})$ and $v_{n}=\left|v_{\mathbf{k}}^{x}\right|+\left|v_{\mathbf{k}}^{y}\right|$ for even $n$, where velocities projections $v_{\mathbf{k}}^{x}$ and $v_{\mathbf{k}}^{y}$ are defined by usual derivatives with respect to corresponding momenta components of the bare electron dispersion $\varepsilon(\mathbf{k})$. At last, $s(n)$ are combinatorial prefactors defining the number of coinciding diagrams:

$$
s(n)=n
$$

for the case of commensurate charge (CDW-type) fluctuations with $\mathbf{Q}=(\pi / a, \pi / a)$ [43]. For incommensurate CDW fluctuations [43] (when $\mathrm{Q}$ is not related to lattice period) we get:

$$
s(n)=\left\{\begin{array}{cc}
\frac{n+1}{2} & \text { for odd } n \\
\frac{n}{2} & \text { for even } n .
\end{array}\right.
$$


If we want to take into account spin (Heisenberg) structure of interaction with spin fluctuations for nearly antiferromagnetic Fermi liquid (spin-fermion model [41]), the combinatorics becomes more complicated. Scattering processes preserving spin projection are controlled by commensurate combinatorics, while spin flip scattering is described by diagrams of incommensurate type ("charged" random field, according to Ref. [41]). In this model the recurrent procedure (16) for single-particle Green's function remains the same but with another combinatorial prefactors $s(n)$ [41]:

$$
s(n)=\left\{\begin{array}{cc}
\frac{n+2}{3} & \text { for odd } n \\
\frac{n}{3} & \text { for even } n .
\end{array}\right.
$$

Obviously this procedure introduces an important length scale $\xi$, missed in standard DMFT. Physically this length scale reflects the influence of short range order fluctuations (SDW or CDW) on electronic bath surrounding effective Anderson impurity in DMFT.

After the self-consistent solution of DMFT $+\Sigma$ set of equations (5-10) is obtained, one can calculate the spectral density $A(\omega, \mathbf{k})$ :

$$
A(\omega, \mathbf{k})=-\frac{1}{\pi} \operatorname{Im} \frac{1}{\omega+\mu-\varepsilon(\mathbf{k})-\Sigma(\omega)-\Sigma_{\mathbf{k}}(\omega)}
$$

where $\Sigma(\omega), \Sigma_{\mathbf{k}}(\omega)$ and chemical potential $\mu$ are already computed in a self-consistent way. Density of states can be found by integration of (21) over the Brillouin zone.

Analogous approach can be developed also to determine two-particle vertices. Basic idea employed here is the possibility to get arbitrary vertex diagram by introducing "external field" line into corresponding self-energy diagram [44, 45, 46]. In the model under consideration we can again restrict ourselves to non-crossing diagrams, while contribution of all other diagrams can be accounted for by combinatorial prefactors $s(n)$ attributed to interaction lines [41, 42, 43]. Thus, all vertex diagrams are obtained from the simple ladder diagrams with additional prefactors $s(n)$ on corresponding interaction lines [45, 46] (see also [29]). Then we obtain the following system of recurrent relations for the vertex $\Gamma^{R A}\left(\varepsilon_{-}, \mathbf{k}_{-} ; \varepsilon_{+}, \mathbf{k}_{+}\right)$[46], where contribution of local DMFT self-energy (obtained within the $\mathrm{DMFT}+\Sigma$ procedure) is already included:

$$
\begin{aligned}
& \Gamma_{n-1}^{R A}\left(\varepsilon_{-}, \mathbf{k}_{-} ; \varepsilon_{+}, \mathbf{k}_{+}\right)=1+\Delta^{2} s(n) G_{n}^{A}\left(\varepsilon_{-}, \mathbf{k}_{-}\right) G_{n}^{R}\left(\varepsilon_{+}, \mathbf{k}_{+}\right) \times \\
& \times\left\{1+\frac{2 i v_{n} \kappa k}{\omega-\varepsilon_{n}\left(\mathbf{k}_{+}\right)+\varepsilon_{n}\left(\mathbf{k}_{-}\right)-\Sigma^{R}\left(\varepsilon_{+}\right)+\Sigma^{A}\left(\varepsilon_{-}\right)-\Sigma_{n+1}^{R}\left(\varepsilon_{+}, \mathbf{k}_{+}\right)+\Sigma_{n+1}^{A}\left(\varepsilon_{-}, \mathbf{k}_{-}\right)}\right\} \times \\
& \times \Gamma_{n}^{R A}\left(\varepsilon_{-}, \mathbf{k}_{-} ; \varepsilon_{+}, \mathbf{k}_{+}\right),
\end{aligned}
$$

where

$$
G_{n}^{R, A}\left(\varepsilon_{ \pm}, \mathbf{k}_{ \pm}\right)=\frac{1}{\varepsilon_{ \pm}-\varepsilon_{n}\left(\mathbf{k}_{ \pm}\right) \pm i n v_{n} \kappa-\Sigma^{R, A}\left(\varepsilon_{ \pm}\right)-\Sigma_{n+1}^{R, A}\left(\varepsilon_{ \pm}, \mathbf{k}_{ \pm}\right)}
$$

"Physical" vertex $\Gamma^{R A}\left(\varepsilon_{-}, \mathbf{k}_{-} ; \varepsilon_{+}, \mathbf{k}_{+}\right)$is defined as $\Gamma_{n=0}^{R A}\left(\varepsilon_{-}, \mathbf{k}_{-} ; \varepsilon_{+}, \mathbf{k}_{+}\right)$. Recurrent procedure (22) accounts for all diagrams of perturbation theory for the vertex part. In the limit of $\kappa \rightarrow 0 \quad(\xi \rightarrow \infty)$ (22) can be reduced to a series investigated in Ref. [44] (see also [41]), which can be exactly summed in analytical form. Standard ladder approximation is reproduced if all combinatorial factors in (22) are made equal to one for all $n$ [45]. Recurrent procedure for $\Gamma^{R R}\left(\varepsilon_{-}, \mathbf{k}_{+} ; \varepsilon_{+}, \mathbf{k}_{+}\right)$differs from (22) only by the evident change of $A \rightarrow R$, as well as replacing the whole expression in figure brackets on the r.h.s. of 
Eq. (22) by 1. Eqs. (41), (16), (22) together with (14) and (13) provide the complete selfconsistent procedure to calculate optical conductivity within our model in the framework of DMFT $+\Sigma$ approach.

Important aspect of our theory is the possibility of microscopic calculation of both effective parameters $\Delta$ and $\xi$. For example, applying two-particle self-consistent theory of Ref. [47, together with approximations introduced in Refs. [41, 42] for two-dimensional Hubbard model, we derived a microscopic expression for $\Delta$ [23], which can be calculated within the standard DMFT. It can be shown that for wide range of hole doping the pseudogap amplitude $\Delta$ varies in the interval from $t$ to $2 t$ ( $t$ is the nearest neighbor hopping integral).

\subsubsection{Basic electronic properties in the pseudogap state.}

Let us discuss results for the standard single band Hubbard model on a square lattice with electron dispersion

$$
\varepsilon(\mathbf{k})=-2 t\left(\cos k_{x} a+\cos k_{y} a\right)-4 t^{\prime} \cos k_{x} a \cos k_{y} a,
$$

with $t$ and $t^{\prime}$ nearest and next nearest hopping integrals.

Energy scale in the following is defined by nearest neighbor hopping integral $t$, and length scale by the lattice constant $a$. Impurity solver used was the numerical renormalization group (NRG) [14, 15]. Detailed computational results on single particle properties demonstrating pseudogap anomalies can be found in Refs. [23, 24, 25], and on optical conductivity in Ref. [26]. Here we only discuss most typical results corresponding mostly to the case of $t^{\prime} / t=-0.4$ (characteristic for cuprates) and band filling $n=0.8$ (hole doping).

\section{Density of states and spectral function.}

Lets start with results obtained within generalized DMFT $+\Sigma$ approach for the densities of states (DOS) in case of rather weak (compared to bandwidth) Coulomb interaction $U=4 t$. Characteristic feature of strongly correlated metallic state is coexistence of lower and upper Hubbard subbands splitted by Coulomb interaction $U$ with quasiparticle peak at the Fermi level [4, 5]. Noninteracting DOS for the square lattice has Van-Hove singularity near the Fermi level, so that in general the peak on the Fermi level can not be treated simply as a quasiparticle one. Actually there are two contributions to this peak: (i) from quasiparticle peak appearing in strongly correlated metals because of manybody effects and (ii) smoothed Van-Hove singularity of noninteracting DOS 3 .

On the left side of Fig. 6 we show DMFT $+\Sigma$ DOS with $n=0.8$ for the case of $t^{\prime} / t=-0.4$ (left panel) and $t^{\prime}=0$ (right panel) for two different temperatures $T=$ $0.356 t$ (lower panel) and $T=0.088 t$ (upper panel). Black curves are obtained in the absence of fluctuations. Other curves on Fig. 6 present results for DOS with nonlocal fluctuations with amplitude $\Delta=2 t$. For all parameter sets we see that introduction of non-local fluctuations leads to pseudogap formation on a quasiparticle peak. Width of the pseudogap (energy interval between corresponding peaks in the DOS) is of the order of $\sim 2 \Delta$. Decrease of the value of $\Delta$ from $2 t$ to $t$ gives twice smaller pseudogap width and makes it less deep. More pronounced pseudogap is found for spin-fermion

\footnotetext{
${ }^{3}$ With decrease of Coulomb repulsion Van-Hove singularity gradually transforms into quasiparticle peak at $U=(6 \div 8) t$.
} 

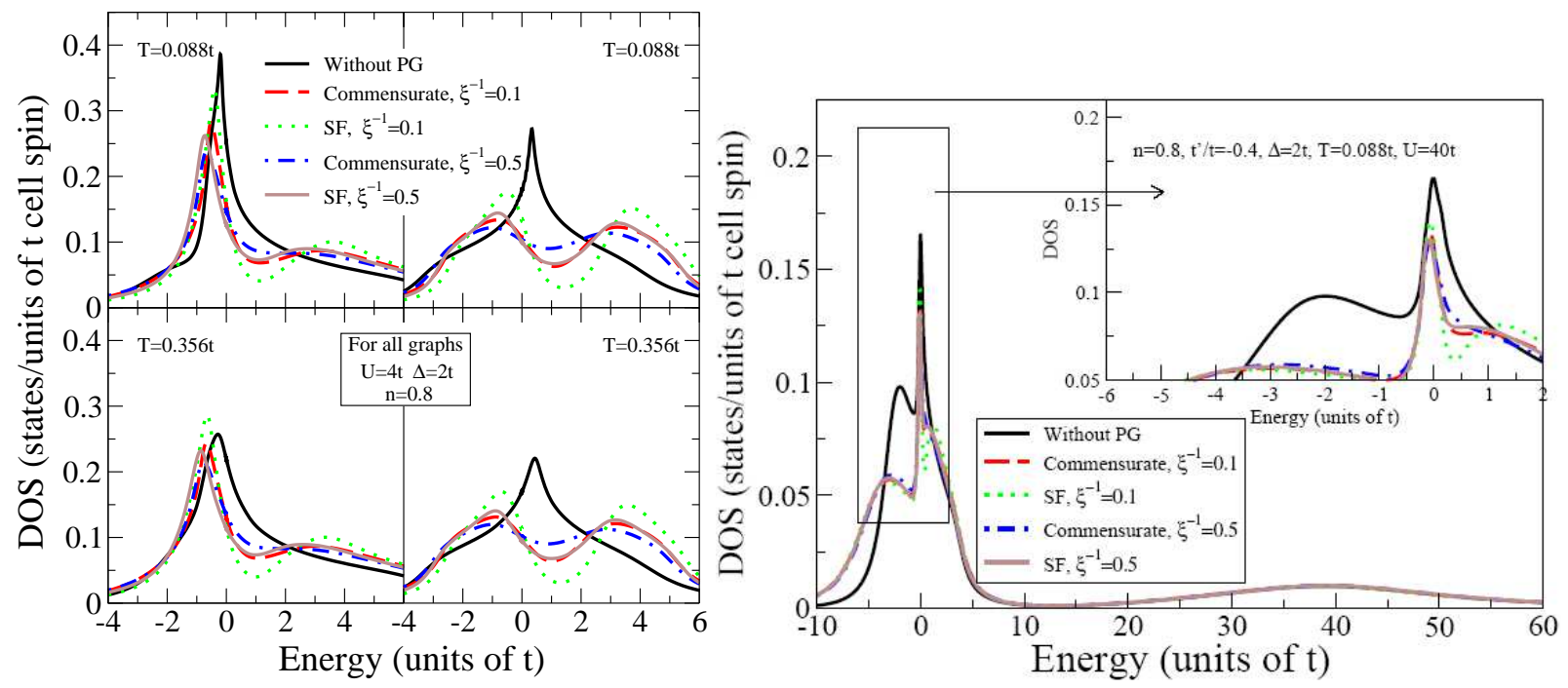

Figure 6: Comparison of DMFT(NRG) $+\Sigma$ DOS calculated in Ref. [23] for different combinatorial prefactors ( $\mathrm{SF}$ - spin-fermion model, commensurate fluctuations), inverse correlation lengths $\xi^{-1}$ (in units of lattice constant), pseudogap amplitude $\Delta=2 t$ and band filling $n=0.8$. On the left side $-U=4 t, t^{\prime} / t=-0.4$ (left column), $t^{\prime}=0$ (right column), temperature $T=0.088 t$ (upper part) and $T=0.356 t$ (lower part). On the right side $-U=40 t, T=0.088 t$.

Fermi level corresponds to zero energy.

model combinatorial prefactors (see (20) ), as compared with the case of commensurate charge fluctuations (combinatorial prefactors (19)). The influence of correlation length value corresponds to general expectations. Decrease of correlation length or, for inverse correlation length, the change from $\xi^{-1}=0.1$ to $\xi^{-1}=0.5$ slightly smears the pseudogap. The rise of temperature from $T=0.088 t$ to $T=0.356 t$ leads to general broadening of DOS structures. One should note that DMFT $+\Sigma$ results for $U=4 t$ (which is less than the bandwidth $W$ ) are qualitatively similar to results obtained earlier in the absence of Hubbard interaction [41, 42].

Let us consider now the case of doped Mott insulator with Hubbard interaction value $U=40 t, t^{\prime} / t=-0.4$ and band filling $n=0.8$ (right side of Fig. 6). Characteristic feature of DOS for such strongly correlated metals is strong splitting of lower and upper Hubbard bands with the Fermi level within the lower Hubbard band (the case of hole doping) In absence of nonlocal fluctuations again the quasiparticle peak is formed on the Fermi level. However upper Hubbard band now is quite far away to the right and does not touch the quasiparticle peak (as it does for the case of weak Hubbard interaction).

For strong enough nonlocal fluctuations with $\Delta=2 t$ pseudogap appears in the middle of quasiparticle peak and the lower Hubbard band is slightly broadened by fluctuations effects. Qualitatively pseudogap anomalies behavior reminds that described above for the case of $U=4 t$ - decrease of $\xi$ smears the pseudogap and makes it less pronounced, decrease of $\Delta$ from $\Delta=2 t$ to $\Delta=t$ narrows the pseudogap and makes it more shallow (see. [23]). Let us notice also that for the doped Mott insulator pseudogap is more evident for spin SDW-like fluctuations than for the charge CDW-like ones.

Nevertheless there are quite appreciable distinctions in contrast to the $U=4 t$ case. For example, the width of the pseudogap in DOS is found to be essentially smaller than $2 \Delta$ which is connected, in our opinion, with noticeable narrowing of the quasiparticle 


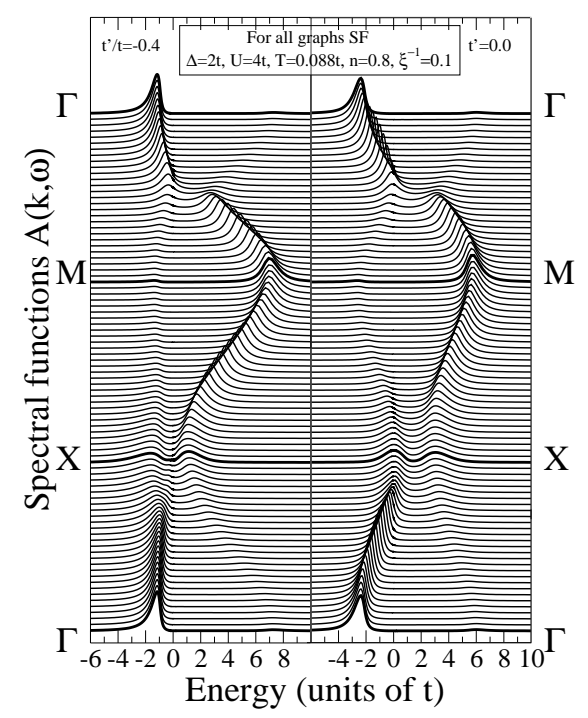

Figure 7: DMFT(NRG) $+\Sigma$ spectral densities $A(\mathbf{k}, \omega)$ [23] along high-symmetry directions of the first Brillouin zone $\Gamma(0,0)-\mathrm{X}(\pi, 0)-\mathrm{M}(\pi, \pi)-\Gamma(0,0)$, for spin-fermion combinatorics (SF). The Fermi level is at zero energy.

peak itself caused by local correlations.

In Fig. 7 we show spectral densities $A(\omega, \mathbf{k})$, calculated within the DMFT $+\Sigma$ approach along high-symmetry directions of the first Brillouin zone: $\Gamma(0,0)-\mathrm{X}(\pi, 0)-$ $\mathrm{M}(\pi, \pi)-\Gamma(0,0)$. In fact this figure shows the quasiparticle band of manybody system - positions of maxima of spectral functions define quasiparticle dispersion, while their width defines quasiparticle damping. Also we clearly observe the partial reconstruction ("destruction") of this band by pseudogap fluctuations. One can see characteristic doublepeak structure close to $X$-point of the Brillouin zone. In the middle of $M-\Gamma$ direction (the so called "nodal"-point) one can observe the rise of the pseudogap, i.e. the "memory" of the AFM gap, which has maximum here in the case of AFM long range order. Generally speaking varying of filling leads to a shift of spectral functions with respect to the Fermi level.

\section{Fermi surface "destruction".}

Within conventional DMFT Fermi surface is not renormalized by interaction i.e. it stays the same as for the bare quasiparticles [3]. However in the case of nontrivial selfenergy momentum dependence substantial renormalization of the Fermi surface appears due to pseudogap formation [41]. There are several ways to define Fermi surface for strongly correlated systems. Below we shall exploit intensity map of the spectral function (spectral density) (21) for $\omega=0$, which is often called the Fermi surface map. Such a map is directly measured by ARPES experiments and positions of its intensity maxima specify the Fermi surface in a sense of the usual Fermi liquid theory, in case of quasiparticle damping becoming negligibly small.

On the right side of the Fig. 8 there are displayed these maps for case of strongly correlated metal with $U=4 t$. This contour plot of the spectral function clearly demonstrates the "destruction" of the Fermi surface in the "hot-spots" together with formation of the 

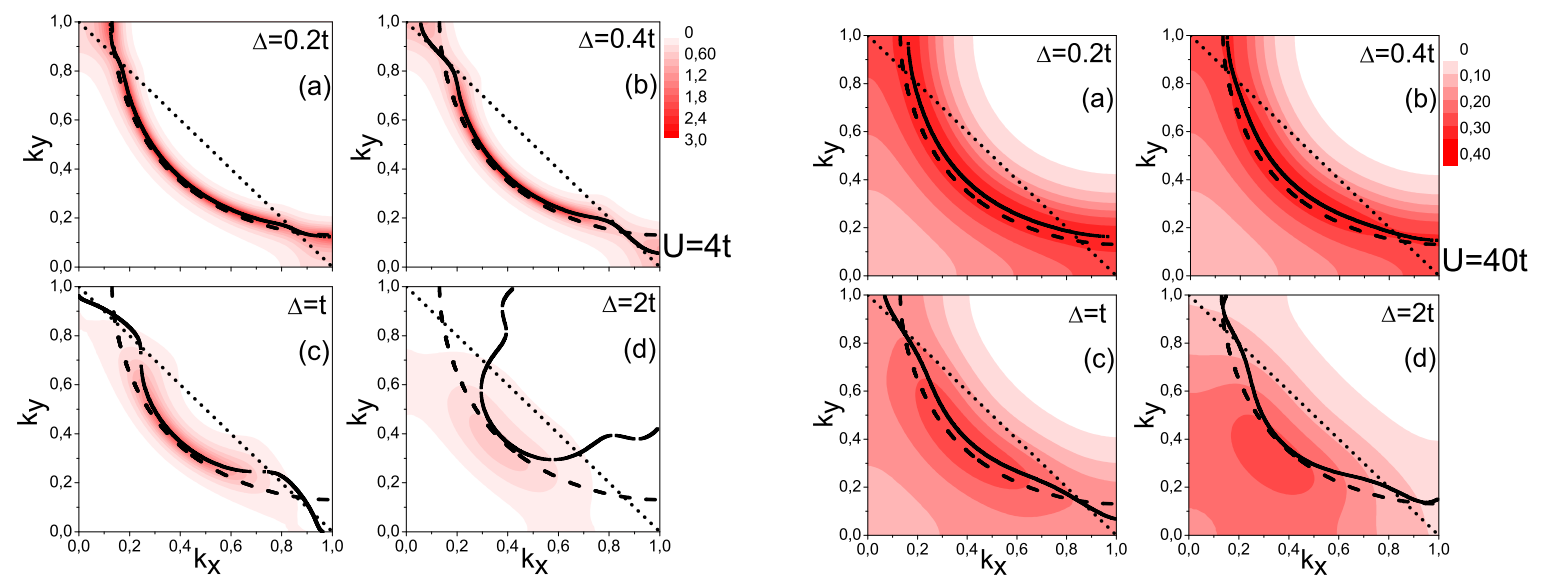

Figure 8: DMFT(NRG) $+\Sigma$ picture of the Fermi surface "destruction" Ref. [24] On the left for $U=4 t$. On the right - for $U=40 t$ (a) $\Delta=0.2 t$; (b) $\Delta=0.4 t$; (c) $\Delta=t$; (d) $\Delta=2 t$. Band filling is $n=0.8$. Dashed line - "bare" Fermi surface. Full lines - solutions of Eq. (25).

"Fermi arcs" upon $\Delta$ growth similar to that observed in pioneer works of Norman et al. [48], which were later confirmed in a large number of other works. One should note that qualitatively analogous behavior is also found in the absence of local electronic correlations $(U=0)$ [24, 49]. The role of finite $U$ values add up to the decrease of spectral function intensity compared to the case $U=0$ and leads to additional "smearing" making "hotspots" less visible. "Destruction" of the Fermi surface starts in the vicinity of "hot-spots" for small $\Delta$ values, but practically simultaneously it disappears in the whole antinodal region (near points $\mathrm{X}(\pi, 0), \mathrm{Y}(0, \pi)$ ) of the Brillouin zone, while only "Fermi arcs" in the nodal region remain, with the shape close to bare Fermi surface. Those results naturally explain why in ARPES the clear "hot-spots" behavior is rather rarely observed [50]. In more details the question of possibility to observe "hot-spots" will be elucidated in the section below, devoted to LDA $+\mathrm{DMFT}+\Sigma$ description of realistic cuprates.

In the case of doped Mott insulator with $U=40 t$ shown in Fig. 8 we see that "Fermi surface" is rather ill defined for all values of $\Delta$. The profile of spectral function is significantly more "smeared" in contrast to smaller values of $U$ reflecting important role of local correlations. For comparison in Fig. 8 we also show the renormalized Fermi surfaces obtained within the model by formal solution of Eq. (25) 4:

$$
\omega-\varepsilon(\mathbf{k})+\mu-\operatorname{Re} \Sigma(\omega)-\operatorname{Re} \Sigma_{\mathbf{k}}(\omega)=0
$$

for $\omega=0$ used for example in the work [41]. Obviously, this definition gives Fermi surface close to the one obtained from intensity map for small $\Delta$, but does not account for significant damping essentially important for large $\Delta$. For large pseudogap amplitudes this definition of the Fermi surface is qualitatively adequate to true behavior, resulting

\footnotetext{
${ }^{4}$ This definition is used in the standard Fermi liquid theory. In fact in our particular case influence of nonlocal pseudogap fluctuations leads to qualitative changes of simple Fermi liquid picture. Herewith we leave aside the question whether Fermi liquid is applicable for such defined Fermi surface or not in the limit $T \rightarrow 0$ since static approximation used is by construction the high temperature one - short range order AFM fluctuations can be considered as quasistatic only if $T \gg \omega_{s f}$, where $\omega_{s f}$ - characteristic frequency of spin fluctuations [1, 42.
} 

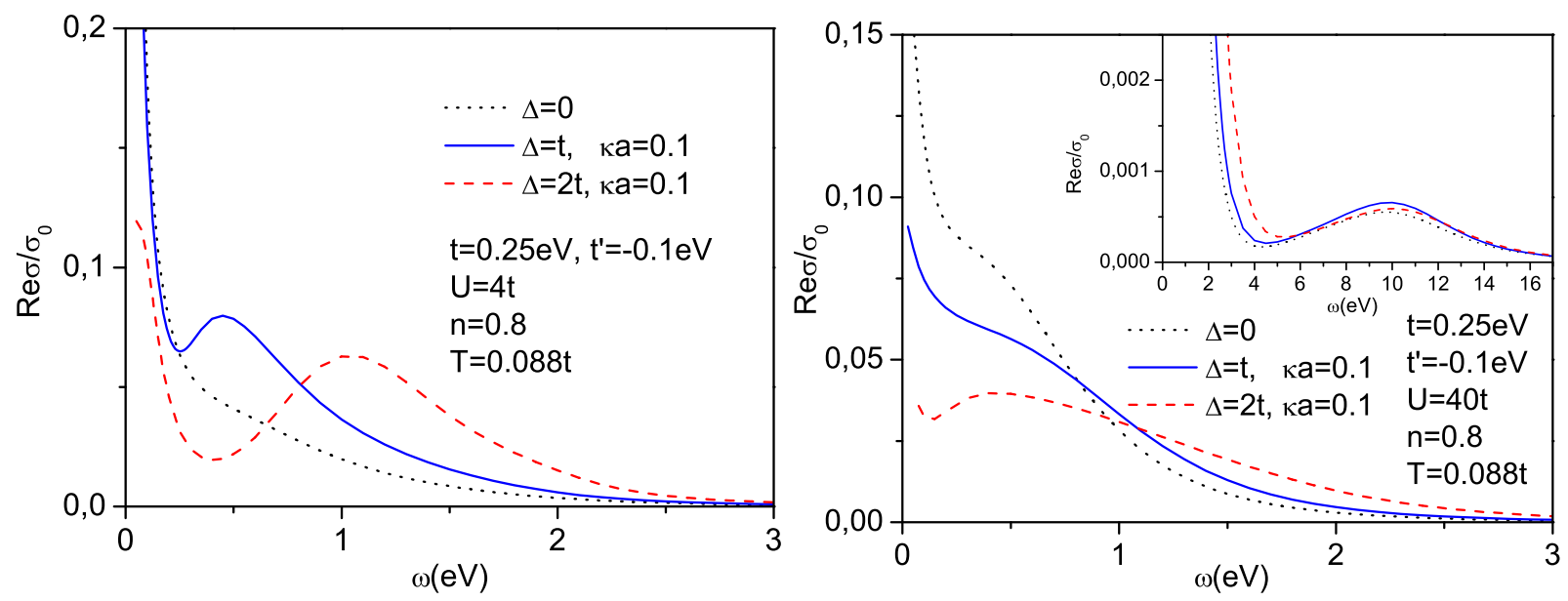

Figure 9: Real part of DMFT $+\Sigma$ optical conductivity $\left(t^{\prime}=-0.4 t, t=0.25 \mathrm{eV}\right)$ obtained in Ref. [26] for different values of the pseudogap amplitude: $\Delta=0, \Delta=t, \Delta=2 t$. Temperature is $T=0.088 t$, band filling $-n=0.8$ and correlation length $\xi=10 a$. On the left side - strongly correlated metal with $U=4 t$. On the right side - doped Mott insulator with $U=40 t$. Inset: conductivity in a wide frequency range, which includes transitions to the upper Hubbard band.

from spectral function analysis, only in the nodal region. Actually, the contour plot of spectral function (at $\omega=0$ ) gives most complete and natural representation of the Fermi surface for the systems with strong correlations and nonlocal fluctuations of some order parameter, which are present in a wide region of the phase diagram of high- $\mathrm{T}_{c}$ cuprates because of their low dimensionality. Results obtained in a such an approach directly correspond to ARPES experiments, where exactly this definition of the Fermi surface is most conventional.

\section{Optical conductivity.}

Lets set about discussion of DMFT $+\Sigma$ results for optical conductivity. On the left panel of Fig. 9 we show DMFT $+\Sigma$ results for the real part of optical conductivity in the case of strongly correlated metal $(U=4 t)$ for different values of the pseudogap amplitude. We clearly observe the formation of typical pseudogap anomaly on the "shoulder" of the Drude peak and it grows as $\Delta$ increases. This behavior is rather similar to "mid-infrared feature" which is observed in optical conductivity of cuprate superconductors [51, 52]. The rise of temperature and decrease of fluctuations correlation length wash off pseudogap, making this anomaly less pronounced [26].

The right panel of Fig. 9 demonstrates DMFT $+\Sigma$ optical conductivity of doped Mott insulator $(U=40 t)$ for several values of the pseudogap amplitude. We see that frequency range where pseudogap anomaly is observed gets narrower with the growth of local correlation strength and for large $U$ values pseudogap anomalies are strongly suppressed. Pseudogap fluctuations lead to noticeable changes of optical conductivity only for relatively low frequencies, of the order of $\Delta$. For higher frequencies (e.g. of the order of $U$, where transitions to upper Hubbard band take place) pseudogap effects are not seen (see also inset on the right panel of Fig. 9). For low frequencies we observe suppression of Drude peak with rather weak anomaly at $\omega \sim \Delta$ which disappears for small $\Delta$ values or for short correlation lengths. 


\subsection{Mott-Anderson transition in disordered systems.}

The importance of both electron interactions and disorder effects in the condensed matter research is well known [53. Coulomb interaction and disorder are two driving forces leading to metal-insulator transition, connected with localization and delocalization of charge carriers. In particular Mott-Hubbard transition is induced by electron repulsion [1, 54, while Anderson metal-insulator transition is related to scattering of noninteracting particles by impurities [55. It is well known that a subtle competition between disorder effects and interaction has many manifestations [53, 56]. Most relevant this problem is in the case of strong disorder and strong electron correlations, determining physical mechanisms of Mott-Anderson metal-insulator transition [53].

One of the main models allowing for the account of both electronic correlations (leading to Mott metal-insulator transition [1, 54]) and strong disorder effects (leading to Anderson metal-insulator transition) is Anderson-Hubbard model [27, 57, 58, 59, 60, 61, 62].

In Refs. [57, 58, 59] three-dimensional Hubbard-Anderson model was investigated in the framework of dynamical mean-field theory (DMFT) [9, 3, 5, 4. Influence of local disorder was taken into account through averaged density of states (DOS) [63, 64] within the well known coherent potential approximation (CPA), which does not describe Anderson localization. To overcome this difficulty in Dobrosavljevic and Kotliar [57] has proposed a version of DMFT, where the self-consistent solution of stochastic DMFT equations for an ensemble of systems with given realizations of disorder, was used to calculate the averaged logarithmic (geometric mean) density of states, which gives information on critical disorder for Anderson transition. Further this approach was developed in Refs. [58, 59] where highly nontrivial phase diagram of three-dimensional paramagnetic Anderson-Hubbard model [59] was obtained, containing correlated metal phase, Mott insulator phase and correlated Anderson insulator phase. Main problem of the approach used in Refs. [57, 58, 59] is the impossibility of direct computation of measurable physical properties such as conductivity, which actually defines metal-insulator transition.

At the same time there exists the well developed self-consistent theory of Anderson localization, based on the solution of equations for the generalized diffusion coefficient. Efficiency of this approach in the absence of interactions is known for a long time [28, 29, 39, 65, 66, 67, certain attempts to include interaction effects into this approach with some promising results were undertaken in Refs. [66, 68. However, up to now this approach was not extended to modern theory of strongly correlated systems. For the first time such investigation was performed in Ref. [27] for three-dimensional systems and later for two-dimensional case [69].

Let us consider disordered paramagnetic Anderson-Hubbard model (mostly) at halffilling for arbitrary interaction and disorder strength. Obviously this model contains both Mott-Hubbard and Anderson metal-insulator transitions. Hamiltonian of the model is:

$$
H=-t \sum_{\langle i j\rangle \sigma} a_{i \sigma}^{\dagger} a_{j \sigma}+\sum_{i \sigma} \epsilon_{i} n_{i \sigma}+U \sum_{i} n_{i \uparrow} n_{i \downarrow},
$$

where $t>0$ is nearest neighbor hopping amplitude, while $U$ is on-site Hubbard repulsion,

$n_{i \sigma}=a_{i \sigma}^{\dagger} a_{i \sigma}$ is particle number operator, $a_{i \sigma}\left(a_{i \sigma}^{\dagger}\right)$ is annihilation (creation) operator of electron on site $i$ with spin $\sigma$. Local energies $\epsilon_{i}$ are assumed to be random and independent at different lattice sites. To simplify diagram technique hereafter we assume the Gaussian 
distribution for $\epsilon_{i}$ :

$$
\mathcal{P}\left(\epsilon_{i}\right)=\frac{1}{\sqrt{2 \pi} \Delta} \exp \left(-\frac{\epsilon_{i}^{2}}{2 \Delta^{2}}\right)
$$

Parameter $\Delta$ here is the measure of disorder. Such Gaussian random field ("white noise") of energy levels $\epsilon_{i}$ at different lattice sites is equivalent to impurity scattering and can be described by standard diagram technique for the averaged Green functions [29].

Self-energy $\Sigma_{\mathbf{p}}(i \varepsilon)$ caused by scattering by disorder calculated in simple one-loop approximation neglecting "crossing" diagrams (i.e. in self-consistent Born approximation) [29], in case the of Gaussian disorder (27) reduces to:

$$
\Sigma_{\mathbf{p}}(i \varepsilon)=\Delta^{2} \sum_{\mathbf{p}} G(i \varepsilon, \mathbf{p}) \equiv \Sigma_{i m p}(i \varepsilon),
$$

so that our "external" self-energy is independent of momentum p (local).

To analyze optical conductivity we shall apply the general DMFT $+\Sigma$ expression (13). Most important block $\Phi_{\varepsilon}^{0 R A}(\omega, \mathbf{q})$ can be obtained using the ideology of self-consistent theory localization [28, 29, 65, 39, 66, 67], with some generalizations to account for the role of Hubbard interaction via DMFT $+\Sigma$ approach [27, 69]. Main distinction from the standard derivation of the equations of self-consistent theory of localization is the use of Green's functions (4) containing local contribution to self-energy from Hubbard interaction.

Following standard derivation [28, 29, 39, 65, 66, 67] we obtain diffusion-like (for small $\omega$ and $q)$ contribution to $\Phi_{\varepsilon}^{0 R A}(\omega, \mathbf{q})$ which takes the form:

$$
\Phi_{\varepsilon}^{0 R A}(\mathbf{q}, \tilde{\omega})=\frac{2 \pi i N(\varepsilon)}{\tilde{\omega}+i D(\omega) q^{2}},
$$

where $D(\omega)$ is the generalized diffusion coefficient and important difference from the single-particle case is contained in:

$$
\tilde{\omega}=\varepsilon_{+}-\varepsilon_{-}-\Sigma^{R}\left(\varepsilon_{+}\right)+\Sigma^{A}\left(\varepsilon_{-}\right)=\omega-\Sigma^{R}\left(\varepsilon_{+}\right)+\Sigma^{A}\left(\varepsilon_{-}\right) \equiv \omega-\Delta \Sigma^{R A}(\omega),
$$

which substitutes for the usual $\omega$ term in the denominator of standard expression for $\Phi_{\varepsilon}^{0 R A}(\omega, \mathbf{q})$. From general considerations it is clear that in metallic phase for $\omega \rightarrow 0$ we have $\Delta \Sigma^{R A}(\omega=0)=2 i \operatorname{Im} \Sigma(\varepsilon) \sim \operatorname{Max}\left\{T^{2}, \varepsilon^{2}\right\}$, which reflects Fermi liquid behavior in DMFT (which is not violated by elastic impurity scattering). For finite $T$ it leads to usual phase decoherence caused by (inelastic) electron-electron scattering [53, 56].

Then Eq. (13) takes the form:

$$
\operatorname{Re} \sigma(\omega)=\frac{e^{2} \omega}{2 \pi} \int_{-\infty}^{\infty} d \varepsilon\left[f\left(\varepsilon_{-}\right)-f\left(\varepsilon_{+}\right)\right] \operatorname{Re}\left\{\frac{2 \pi N(\varepsilon) D(\omega)}{\omega^{2}}-\phi_{\varepsilon}^{0 R R}(\omega)\left[1-\frac{\Delta \Sigma^{R R}(\omega)}{\omega}\right]^{2}\right\},
$$

where the second term in figure brackets can be in fact neglected for small $\omega$, while in case of necessity to describe a wide frequency range it can be calculated using (14) with $\Phi_{\varepsilon}^{0 R R}(\omega, \mathbf{q})$ taken in the usual ladder approximation.

Now following the standard scheme of self-consistent theory of localization [28, 29, 39, 65. 66, 67] we get the closed self-consistent equation for generalized diffusion coefficient:

$$
D(\omega)=i \frac{<v>^{2}}{d}\left\{\tilde{\omega}-\Delta \Sigma_{i m p}^{R A}(\omega)+\Delta^{4} \sum_{\mathbf{p}}\left(\Delta G_{\mathbf{p}}\right)^{2} \sum_{\mathbf{q}} \frac{1}{\tilde{\omega}+i D(\omega) q^{2}}\right\}^{-1}
$$


where $d$ is spatial dimensionality, $\Delta G_{\mathbf{p}}=G^{R}\left(\varepsilon_{+}, \mathbf{p}\right)-G^{A}\left(\varepsilon_{-}, \mathbf{p}\right), \Delta \Sigma_{i m p}^{R A}(\omega)=\sum_{\text {imp }}^{R}\left(\varepsilon_{+}\right)-$ $\Sigma_{i m p}^{A}\left(\varepsilon_{-}\right)$and averaged velocity $\langle v\rangle$, which can be well approximated just by the Fermi velocity, is given by the following expression:

$$
<v>=\frac{\sum_{\mathbf{p}}\left|\mathbf{v}_{\mathbf{p}}\right| \Delta G_{\mathbf{p}}}{\sum_{\mathbf{p}} \Delta G_{\mathbf{p}}}
$$

where $\mathbf{v}_{\mathbf{p}}=\frac{\partial \epsilon(\mathbf{p})}{\partial \mathbf{p}}$. Eq. (32) should be solved together with self-consistent DMFT $+\Sigma$ procedure (510, 28). In fact, this equation is a transcendental one and can be easily solved by iterations for each $\tilde{\omega}$ value.

In accordance with the usual applicability limit of diffusion approximation, summation over $q$ in Eq. (32) should be restricted to [66, 29]:

$$
q<k_{0}=\operatorname{Min}\left\{l^{-1}, p_{F}\right\}
$$

where $l=\langle v>/ 2 \gamma$ is an elastic mean free path, $\gamma$ is Born scattering frequency by impurities, $p_{F}$ is the Fermi momentum. It is well known that in two-dimensional case Anderson localization occurs for any infinitely weak disorder. However, in this case the localization radius is exponentially large and sample size becomes essentially important. Sample size L can be introduced into self-consistent localization theory introducing the integration cutoff of diffusion pole at small $q$ [28, 65], i.e. at:

$$
q \sim k_{L}=1 / L
$$

For $\omega \rightarrow 0$ (on the Fermi surface $(\varepsilon=0$ ), and obviously $\tilde{\omega} \rightarrow 0$ ) in the Anderson insulator phase one gets localization behavior of the generalized diffusion coefficient [28, 65, 29]:

$$
D(\omega)=-i \tilde{\omega} R_{l o c}^{2}
$$

After substitution of (36) into (32) one obtains equation defining localization radius $R_{\text {loc }}$ :

$$
R_{l o c}^{2}=-\frac{<v>^{2}}{d \Delta^{4}}\left\{\sum_{\mathbf{p}}\left(\Delta G_{\mathbf{p}}\right)^{2} \sum_{\mathbf{q}} \frac{1}{1+R_{l o c}^{2} q^{2}}\right\}^{-1} .
$$

\subsubsection{Three-dimensional systems.}

Below we present most interesting results for three-dimensional Anderson-Hubbard model at half-filling on a cubic lattice with semielliptic bare density of states with the bandwidth $W=2 D$ :

$$
N_{0}(\varepsilon)=\frac{2}{\pi D^{2}} \sqrt{D^{2}-\varepsilon^{2}}
$$

Density of states is given in units of number of states in energy interval for unit cell of the volume $a^{3}$ ( $a$ is the lattice constant) and for one spin projection. Conductivity values are given in natural units of $e^{2} / \hbar a$ ( $a$ is the lattice constant). For more detailed acquaintance with numerical results (also for the case of deviations from half-filling) we refer the reader to Ref. [27].

\section{Evolution of the density of states}

Within the standard DMFT approach Hubbard model density of states at half-filling has a typical three-peak structure [5, 4, 170] with a narrow quasiparticle peak (central 

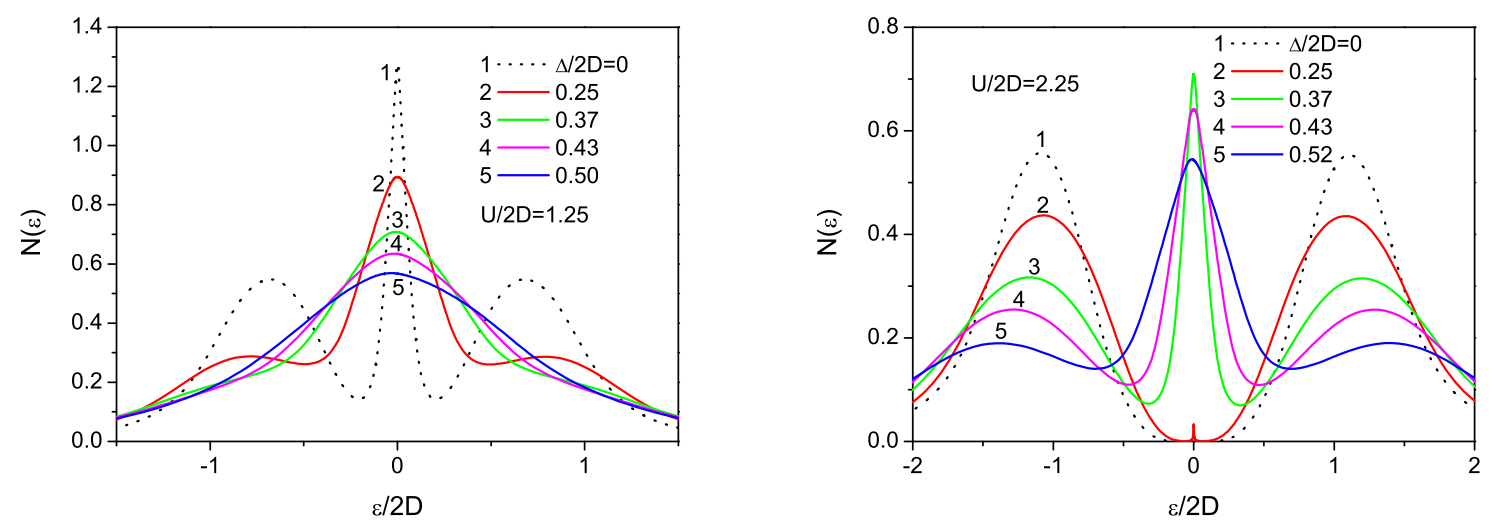

Figure 10: Hubbard-Anderson model density of states at half-filling for different disorder levels $\Delta$ [27]. On the left side - correlated metal with $U=2.5 D$, On the right side - Mott insulator with $U=4.5 D$.

peak) at the Fermi level and wide upper and lower Hubbard bands situated at energies $\varepsilon \sim$ $\pm U / 2$. As $U$ grows quasiparticle band narrows within the metallic phase and disappears at Mott-Hubbard metal-insulator transition at critical interaction value $U_{c 2} \approx 1.5 \mathrm{~W}$. With further increase of $U$ insulating gap opens at the Fermi level.

In Fig. 10 we present our results for DMFT $+\Sigma$ densities of states for typical strongly correlated metal with $U=2.5 D=1.25 \mathrm{~W}$, in the absence of disorder and for different values of disorder $\Delta$, including strong enough disorder, transforming correlated metal to correlated Anderson insulator (see also next section on conductivity). As one can expect disorder leads to typical broadening and suppression of the density of states.

More unexpected is the result obtained for $U=4.5 \mathrm{D}=2.25 \mathrm{~W}$, typical for Mott insulator and shown on right panel of Fig. 10. Here we observe restoration of the central peak (quasiparticle band) in DOS with the increase of disorder, transforming Mott insulator to correlated metal or to correlated Anderson insulator. Similar DOS behavior was reported also in Ref. [59].

Physical origin of such quite unexpected central peak restoration is pretty clear. Controlling parameter of metal-insulator transition in DMFT is the ratio of Hubbard interaction $U$ to bare bandwidth $W=2 D$. With disordering (in the absence of Hubbard interaction) new effective bandwidth $W_{\text {eff }}$ appears which grows with disorder. Semielliptic form of DOS with well defined band edges within the self-consistent Born approximation (28) is preserved. This leads to diminishing values of the ratio $U / W_{\text {eff }}$, which in its turn causes restoration of the quasiparticle band. In more details this is discussed below, when we discuss the phase diagram of Hubbard-Anderson model.

In absence of disorder characteristic feature of Mott-Hubbard metal-insulator transition is hysteresis DOS behavior appearing with the decrease of $U$ starting from insulating phase [5, 70]. Mott insulator phase is conserved (metastable) down to rather small $U$ values deep within the correlated metal phase. Metallic phase is restored only at about $U_{c 1} \approx 1.0 \mathrm{~W}$. Corresponding interval $U_{c 1}<U<U_{c 2}$ typically is considered as a coexistence region of metallic and Mott insulating phases, where, from a thermodynamic point of view, metallic phase is more stable [5, 70, 71]. Such hysteresis DOS behavior (see 

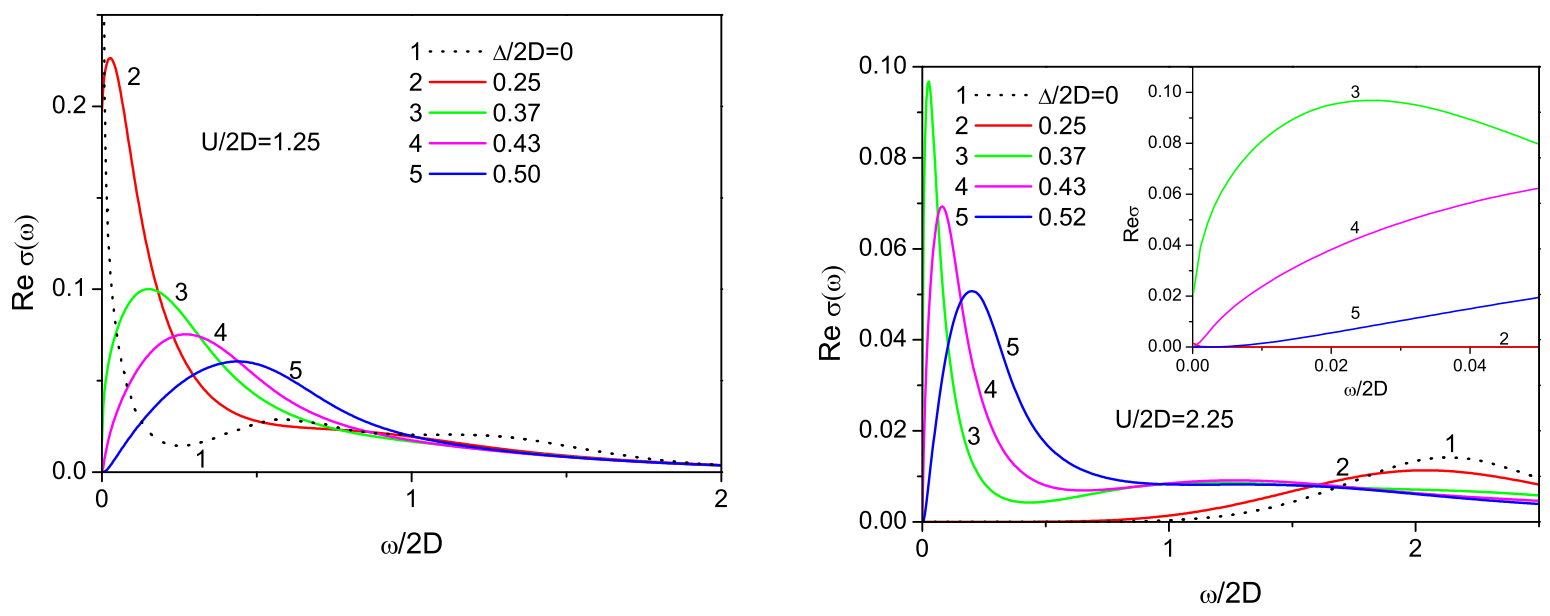

Figure 11: Real part of optical conductivity of Hubbard-Anderson model at half-filling for different disorder levels $\Delta$ [27]. On the left side - typical correlated metal with $U=2.5 D$. Curves 1,2, - metallic phase, curve 3 corresponds to the mobility edge (Anderson transition), curves 4,5 - correlated Anderson insulator. On the right side typical Mott insulator with $U=4.5 D$. Curves 1,2 correspond to Mott insulator, curve 3 - mobility edge (Anderson transition), curves 4,5 - correlated Anderson insulator. Inset - magnified low frequency region.

[27]) is observed also in the presence of disorder and will be described below during the discussion of the phase diagram of Anderson-Hubbard model.

\section{Optical conductivity: Mott-Hubbard and Anderson transitions}

Without disorder our calculations reproduce conventional DMFT results [4, 5], where optical conductivity is characterized by the usual Drude peak at low frequencies and wide maximum at about $\omega \sim U$, which corresponds to optical transitions to upper Hubbard band. As $U$ grows Drude peak diminishes and disappears at Mott transition. Introduction of disorder leads to qualitative change of the frequency dependence of optical conductivity.

On the left panel of Fig. 11 we show the real part of optical conductivity of HubbardAnderson model at half-filling for different disorder levels $\Delta$ and $U=2.5 D$ typical for correlated metal. Transitions to the upper Hubbard bands at energies $\omega \sim U$ are almost unobservable. However it is clearly visible that metallic Drude peak typically centered at zero frequency is broadened and suppressed by disorder, gradually transforming into a peak at finite frequency because of Anderson localization effects. Anderson transition takes place at $\Delta_{c} \approx 0.74 D=0.37 \mathrm{~W}$ (corresponding to the curve 3 on all figures here and also for DOS). Notice that this value depends on the cutoff (34), which is defined up to the coefficient of the order of unity [66, 29]. Naive expectations can bring us to a conclusion that narrow quasiparticle band at the Fermi level (formed in a strongly correlated metal) may be localized much easily than normal conduction band. However we see that these expectations are wrong and the band localizes only at rather large disorder $\Delta_{c} \sim D$, similar to that for conduction band of the width $\sim W$. It agrees with the known analysis of localization in a two-band model [72].

In the DMFT $+\Sigma$ approach critical disorder value $\Delta_{c}$ does not depend on $U$ as interaction effects enter Eq. (32) only through $\Delta \Sigma^{R A}(\omega) \rightarrow 0$ for $\omega \rightarrow 0$ (for $T=0, \varepsilon=0$ ), and the influence of interaction at $\omega=0$ disappears. In fact this is the main shortcoming 
of DMFT $+\Sigma$ approach originating from the neglect of interference effect between interaction and impurity scattering. Significant role of these interference effects is known for a long time [53, 56]. On the other hand, the neglect of these effects allows to perform the reasonable physical interpolation between two main limits - that of Anderson transition because of disorder and Mott-Hubbard transition because of strong correlations. One can consider this approximation as a reasonable first step to a future complete theory of metal-insulator transition in strongly correlated disordered systems.

On the right panel of Fig. 11 we show the real part of optical conductivity of MottHubbard insulator with $U=4.5 D$ at different disorder levels $\Delta$. In the inset we show low frequency data, demonstrating different types of conductivity behavior, especially close to Anderson transition and within the Mott insulator phase. On the main part of the figure contribution to conductivity from transitions to upper Hubbard band at about $\omega \sim U$ is distinctly seen. Disorder growth results in the rise of finite conductivity for the frequencies inside Mott-Hubbard gap, correlating with the restoration of quasiparticle band in DOS within the gap as shown in the right panel of Fig. 10, This conductivity for $\Delta<\Delta_{c}$ is metallic (finite in the static limit $\omega=0$ ), and for $\Delta>\Delta_{c}$ at low frequencies we get $\operatorname{Re} \sigma(\omega) \sim \omega^{2}$, which is typical for Anderson insulator [28, 29, 39, 65, 66, 67].

A bit unusual is the appearance in $\operatorname{Re} \sigma(\omega)$ of a peak at finite frequencies even in the metallic phase. This happens because of importance of localization effects. In the "ladder" approximation for $\Phi_{\varepsilon}^{0 R A}(\omega, \mathbf{q})$ which neglects all localization effects we obtain the usual Drude peak at $\omega=0$ [27], while account of localization effects shifts the peak in $\operatorname{Re} \sigma(\omega)$ to a low (finite) frequencies. As is well known [1], metallic state is defined by finite static $(\omega=0)$ conductivity at zero temperature.

Above we presented the data for conductivity data obtained for the case of increase of $U$ from metallic to Mott insulator phase. As $U$ decreases from Mott insulator phase we observe hysteresis of conductivity in coexistence region defined (in the absence of disorder) by inequality $U_{c 1}<U<U_{c 2}$. Hysteresis of conductivity is also observed in the coexistence region in the presence of disorder. Details of this behavior of optical conductivity can be found in Ref. [27].

\section{Phase diagram of Anderson-Hubbard model at half-filling}

Phase diagram of Anderson-Hubbard model at half-filling was studied in Ref. [59], using direct DMFT calculations for the lattice with finite number of sites with random realizations of energies $\epsilon_{i}$ in (26) and averaging over these realizations to get averaged DOS and geometric mean local DOS which allows one to define critical disorder for transition into Anderson insulator phase. Below we present our results on Anderson-Hubbard model phase diagram obtained from DOS and optical conductivity calculations within the DMFT $+\Sigma$ approach. One should emphasize that conductivity analysis is most direct way to distinguish metallic and insulating phases [1].

Calculated disorder-correlation $(\Delta, U)$ phase diagram at zero temperature is shown on the left panel of Fig. 12. Anderson transition line $\Delta_{c} \approx 0.37 W=0.74 D$ is defined as a disorder strength for which static conductivity becomes zero at $T=0$. Mott-Hubbard transition can be detected from central (quasiparticle) peak disappearance in DOS or from optical conductivity by observation of gap closing in the insulating phase or from static conductivity disappearance in the metallic phase.

We have already noticed that DMFT $+\Sigma$ approximation gives universal ( $U$ independent) value of critical disorder $\Delta_{c}$ because of neglect of interference between disorder scattering and Hubbard interaction. This leads to differences between phase diagram of 

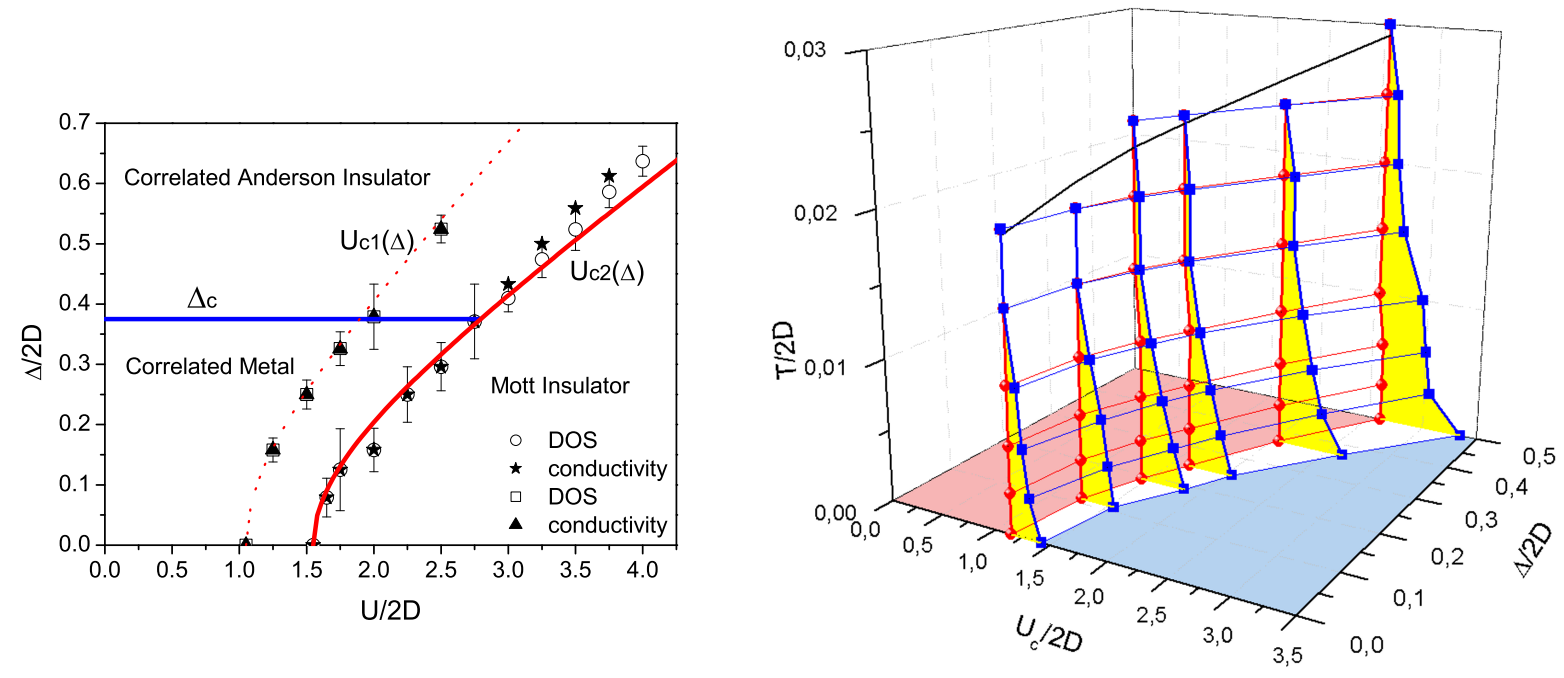

Figure 12: Phase diagram of paramagnetic Anderson-Hubbard model. On the left side zero temperature case [27]. Continuous curves are Mott insulator phase boundaries $U_{c 1, c 2}(\Delta)$ obtained from analytical estimate of Eq. (41), different symbols represent results for these boundaries obtained from calculations from DOS and optical conductivity. Line of Anderson transition is given by $\Delta_{c}=0.37$. On the right side - finite temperature case. Points are obtained from DOS calculations. Solid black curve is linear fit $\left(T_{c} / 2 D=0.02(1+\Delta / 2 D)\right)$ to the $T_{c}$ points where coexistence region disappears.

Fig. 12 and the one obtained in Ref. [59]. At the same time influence of disorder scattering on Mott-Hubbard transition is highly nontrivial and qualitatively coincide with results of Ref. [59]. Main difference is conservation of Hubbard bands in our results even in the limit of high enough disorder, while in the Ref. [59] they just disappear. Moreover coexistence region in Fig. 12 slowly widens with disorder growth instead of vanishing at some "critical" point as on phase diagram of Ref. [59]. Coexistence boundary regions, which are defined by Mott insulator phase boundaries, obtained with decrease on increase of $U$, represented by curves $U_{c 1}(\Delta)$ and $U_{c 2}(\Delta)$ on Fig. 12, can be obtained from the simple equation:

$$
\frac{U_{c 1, c 2}(\Delta)}{W_{\text {eff }}}=\frac{U_{c 1, c 2}}{W}
$$

where effective bandwidth in the presence of disorder was calculated for $U=0$ within self-consistent Born approximation (28):

$$
W_{e f f}=W \sqrt{1+16 \frac{\Delta^{2}}{W^{2}}}
$$

Thus the boundaries of coexistence region which define also Mott insulator phase boundaries are given by:

$$
U_{c 1, c 2}(\Delta)=U_{c 1, c 2} \sqrt{1+16 \frac{\Delta^{2}}{W^{2}}}
$$

which are shown in Fig. 12 by dotted and solid lines. Phase transition points detected from disappearance of quasiparticle peak as well as points following from qualitative changes of conductivity behavior are shown in Fig. 12 by different symbols. These symbols demonstrate very good agreement with analytical results supporting the choice of ratio (39) as a control parameter of Mott transition in presence of disorder. 
On the right panel of Fig. 12 we show temperature dependence of Mott insulator phase boundaries $U_{c 2}(\Delta)$ (squares) and coexistence region $U_{c 1}(\Delta)$ (circles) 5 . It is well known that in "pure" DMFT without disorder coexistence region narrows with temperature growth and vanishes at some critical temperature $T_{c}$. In the DMFT $+\Sigma$ approach disorder increase at zero temperature widens the coexistence region and this behavior remains for finite temperatures. It is also seen that disorder growth leads to a practically linear growth (solid black line) of this critical temperature. Notice also very weak temperature dependence of coexistence boundary $U_{c 1}(\Delta)$.

\subsubsection{Two-dimensional systems.}

According to scaling theory of localization [73] metallic state in two-dimensional (2D) systems does not exists, electrons are localized already at any infinitely small disorder. Despite this prediction for 2D systems was made for noninteracting particles, later it was found that in the simplest case weak interaction between electrons also favors localization [74]. In early 80th experiments done on different 2D systems [75] mostly confirmed these predictions. However, some theoretical works [56] pointed that this point of view in general is incorrect, since in the limits of weak disorder and large enough interaction 2D systems can have finite conductivity at zero temperature. Experimental discovery of metal-insulator transition in 2D weakly disordered systems at low carrier concentration, absent in the single particle theory, stimulated new direction of theoretical studies (see introduction to this field in review papers [76, 77]).

In DMFT $+\Sigma$ approximation, as we shall see, for infinite $2 \mathrm{D}$ system $(L \rightarrow \infty)$ localization radius defined by Eq. (37) remains finite (but exponentially large) for any infinitely weak disorder, signalling the absence of Anderson transition in such system, similarly to the case of the usual single particle theory. However, as we shall discover below, localization radius in finite size systems diverges at some critical disorder, which is defined by the system size L. Qualitatively, this critical disorder is determined by the condition that localization radius of infinitely large system becomes comparable to characteristic sample size $R_{l o c}^{L \rightarrow \infty} \sim L$. Thus for finite two-dimensional systems Anderson transition in fact exists, as well as metallic phase for disorder below some critical value. In the following, under the term "correlated metal" phase we shall imply precisely such phase for finite 2D systems.

Below we discuss most relevant results of $\mathrm{DMFT}+\Sigma$ approach for $2 \mathrm{D}$ AndersonHubbard model at half-filling on a square lattice with rectangular bare density of states with the bandwidth $W=2 D$ :

$$
N_{0}(\varepsilon)=\left\{\begin{array}{ll}
\frac{1}{2 D} & |\varepsilon| \leq D \\
0 & |\varepsilon|>D
\end{array} .\right.
$$

which corresponds right to a $2 \mathrm{D}$ case.

\section{Density of states and optical conductivity.}

Calculations show that qualitative behavior of the density of states in 2D is completely analogous to that discussed above in three-dimensional case. Some quantitative distinctions are due to the different model of the "bare" density of states (42), leading in particular to larger (than in three-dimensional case) critical Hubbard interaction $U_{c 2} \approx 1.83 \mathrm{~W}$, corresponding to Mott metal-insulator transition in absence of disorder

\footnotetext{
${ }^{5}$ These results for different temperatures were calculated by N.A. Kuleeva.
} 
and $U_{c 1} \approx 1.42 \mathrm{~W}$, which limiting below the region coexistence of metallic and insulating phases. Similar to three-dimensional model for $U>U_{c 2}$ (i.e. for Mott insulator without disorder) increase of disorder leads to restoration of quasiparticle peak in the density of states. However, in this case such behavior does not signal in general the transition to a correlated metal state, as for infinitely large systems we are dealing here with correlated Anderson insulator.

Optical conductivity behavior in a wide frequency range is also qualitatively is quite similar to that in three-dimensional model. But for infinite 2D model zero frequency conductivity always disappears (in the zero temperature limit) and, in contrast to $d=$ 3 case [27, even at very weak disorder the peak in optical conductivity lies at finite frequency. In the ladder approximation, which does not contain localization corrections, the usual Drude peak is observed at zero frequency and conductivity at $\omega=0$ is finite.

In more detail results for DOS and optical conductivity in $2 \mathrm{D}$ model can be found in Ref. [69].

\section{Localization radius and phase diagram of 2D Anderson-Hubbard model at half-filling.}

On the left side of Fig. 13 we show dependence of conductivity on disorder $\Delta$ for a finite but quite low frequency $\omega=0.00005 D$. Circles show results of ladder approximation, triangles - self-consistent theory of localization. Curve 3 qualitatively coinciding with ladder approximation was obtained from classical Drude expression:

$$
\sigma(\omega)=\sigma(0) \frac{\gamma^{2}}{\gamma^{2}+\omega^{2}},
$$

where static conductivity $\sigma(0)=e^{2} N(0) D_{0} \approx \frac{e^{2}}{\hbar} \frac{\varepsilon_{F}}{2 \pi \gamma}, N(0)$ - density of states at the Fermi level, $D_{0}$ is Drude diffusion coefficient. Impurity scattering rate was taken as $\gamma=\pi N(0) \Delta^{2} \approx \frac{\pi}{2 D} \Delta^{2}$. Significant contribution from localization corrections to conductivity at finite frequency (noticeable distinction of curve 2 from 1 and 3) appears only when conductivity reaches values of the order of minimal metallic conductivity $\sigma_{0}=\frac{e^{2}}{\hbar}$ (which is taken as unit of conductivity on figures). One should note that exactly in this range of disorder, as we shall see below, Anderson metal-insulator transition takes place (localization radius diverges) for 2D systems of reasonable finite sizes.

Also in Fig. 13 we show dependences of localization radius logarithm following from (37) (right scale) as function of disorder: curve 1 - infinite sample, curves 2 and 3 are for the finite size samples with $L=10^{8} a$ and $L=10^{5} a$ correspondingly. It is seen that localization radius grows exponentially as disorder diminishes and remains finite in the infinite 2D system, where Anderson transition is absent. For the finite systems localization radius diverges at critical disorder determined by system size, demonstrating the existence of an effective Anderson transition. As can be seen from Fig. 13, qualitatively the critical disorder is defined by condition that localization radius of infinite system becomes comparable with characteristic sample size $R_{l o c}^{L \rightarrow \infty} \sim L$. It should be noted that within our approach localization radius practically does not depend on $U$ (in contrast to e.g. [61]), which leads to independence of critical disorder of correlation strength $U$ in $2 \mathrm{D}$ finite size systems. Analogous situation is realized also for three-dimensional systems [27]. In general, it is of course a drawback of our approximations.

On the same left part of Fig. 13 we plot the dependence of static conductivity on disorder strength in finite samples with sizes $L=10^{8} a$ and $L=10^{5} a$ (curves 4 and 5 correspondingly). In the finite size systems with weak disorder static conductivity is 

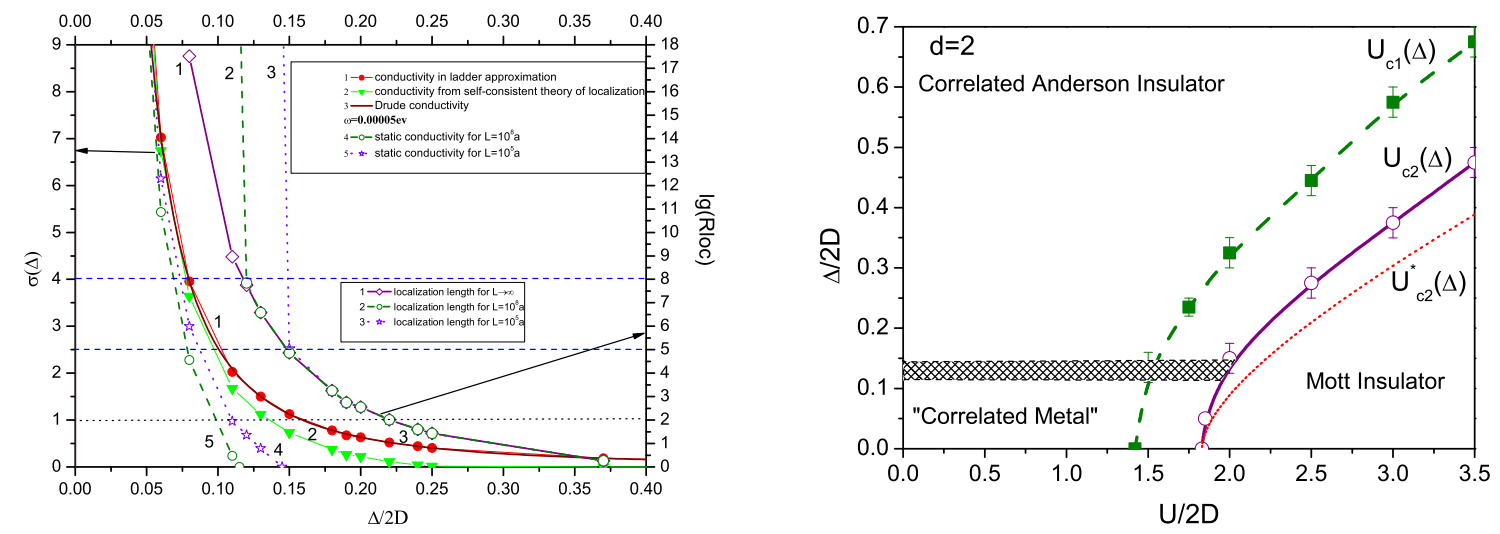

Figure 13: ON the left side - low finite frequency $(\omega=0.00005 D)$ conductivity dependence on disorder strength $\Delta$ at $U / 2 D=1$. Circles (curve 1) show results of ladder approximation, triangles (curve 2) - self-consistent theory of localization. Curve 3 (practically coinciding with ladder approximation) is obtained from Drude formula (43). Curves 4 and 5 - static conductivity of finite samples with sizes $L=10^{8} a$ and $L=10^{5} a$ correspondingly. On the right scale localization radius logarithm versus disorder strength $\Delta$ : infinite size sample - curve 1 , finite samples with sizes $L=10^{8} a$ and $L=10^{5} a$ - curves 2 and 3 69.

On the right - phase diagram of $2 \mathrm{D}$ paramagnetic Anderson-Hubbard model at zero temperature 69. Mott insulator region boundary $U_{c 2}(\Delta)$ and coexistence region boundary $U_{c 1}(\Delta)$ were obtained from density of states behavior. Hatched part - region of an effective Anderson metalinsulator transition in finite size systems.

not zero (metal) and gradually goes down with increase of disorder. It becomes zero at critical disorder, where localization radius diverges in the sample of corresponding size. Static conductivity of finite size samples within our approximation does not practically depend on correlation strength $U$. Significant difference of static conductivity from low finite frequency conductivity observed in Fig. 13 is related to exponential smallness of the frequency range with localization behavior of conductivity, mentioned above.

Let us consider now the phase diagram of 2D paramagnetic Anderson-Hubbard model at half-filling, obtained from DMFT $+\Sigma$ calculated densities of states and from the analysis of localization radius behavior in finite size 2D systems. Such phase diagram in coordinates disorder of $\Delta$ and correlation strength $U$ is shown on the right part of Fig. 13.

Hatched stripe corresponds to the region of effective "metal"- Anderson insulator transition. Boundaries of this region are defined by divergence of localization radius in finite samples with characteristic sizes $L=10^{5} a$ (upper boundary) and $L=10^{8}$ a (lower boundary) (see left side of Fig. 13). One should emphasize that further increase of the system size, e.g. ten times, up to $L=10^{9} a$, leads only to quite insignificant drop of critical disorder. In other words, it slightly shifts down low boundary of hatched stripe (on the right part of Fig. 13) - characteristic region of an effective Anderson transition in finite size systems.

Curve $U_{c 2}(\Delta)$ computed from density of states behavior defines the boundary of Mott transition. Transition criteria is the disappearance of central quasiparticle peak in the density of states $N(\varepsilon)$ together with gap opening on the Fermi level. Similarly to three- 
dimensional model, decrease of $U$ starting from insulating phase leads to Mott transition $U=U_{c 1}(\Delta)<U_{c 2}(\Delta)$ and coexistence (hysteresis) region is observed on the phase diagram between curves $U_{c 1}(\Delta)$ and $U_{c 2}(\Delta)$ (Fig. 13). In analogy with three-dimensional case we can guess that the ratio of Hubbard interaction and effective bandwidth $\frac{U_{c 1, c 2}(\Delta)}{W_{e f f}(\Delta)}$ controls Mott metal-insulator transition and is a universal constant, which does not depend on disorder and obtain qualitative dependencies $U_{c 2}^{*}(\Delta)$ for $2 \mathrm{D}$ model, which is plotted by dotted curve on Fig. 13. It is seen that in contrast to $d=3$ case [27] the dependence of $U_{c 2}(\Delta)$ obtained from straightforward calculations of densities of states significantly differs from the qualitative $U_{c 2}^{*}(\Delta)$ dependence. Apparently it is related to important change of density of states lineshape (at $U=0$ ) as disorder $\Delta$ grows, which is absent for semielliptic band in $d=3$ case.

\subsection{Singularities of electron dispersion in strongly correlated systems in DMFT and DMFT $+\Sigma$ approaches.}

\subsubsection{Cusps ("kinks") in electron spectra.}

Electronic properties of crystalline solids are determined by single-particle and collective excitations of electron subsystem and their interactions with each other. These excitations are characterized by energy $E$ and momentum $\mathbf{k}$ which are related to each other via dispersion (spectrum) $E_{\mathbf{k}}$. Interaction between single-particle and collective modes can result in noticeable bends (cusps) of dispersion $E_{\mathbf{k}}$ - the so called "kinks". The lineshape and energy position of these features characterize interactions in the manybody system. For example, kinks in electron dispersions experimentally observed by ARPES in copper oxides, with energies 40-70 meV below the Fermi level, are evidence of electron-phonon [78, 79, 80, 81, 82, 83, 84] or electron-magnon (spin-fluctuation) interactions [85, 86].

At the same time ARPES experiments detect kinks in electron dispersion for a number of different systems at essentially higher energies (up to $800 \mathrm{meV}$ ) [87, 88, 89]. Physical nature of these kinks remains unknown. Among other attempts to find the explanation of these electron spectra anomalies a novel purely electronic mechanism of kinks formation was proposed in Ref. [90]. This mechanism is applicable to strongly correlated metals, where spectral function contains well developed Hubbard subbands, together with central quasiparticle peak as, for example, in transition metal oxides. Energy location of these kinks apparently determines the range of applicability of Landau Fermi liquid theory.

As is well known [91] in general case interaction results in finite life time of excitations in the system, so that $E_{\mathbf{k}}$ becomes a complex function. For electron systems with Coulomb interaction Landau Fermi liquid theory proves the existence of weakly damped fermionic quasiparticles for low enough temperature and in the narrow energy interval around the Fermi surface [91]. Beyond the Fermi liquid regime the concept of quasiparticles with well defined dispersion, strictly speaking, is not applicable since quasiparticle lifetime is too small. However, in recent years ARPES experiments convincingly show the existence of essentially k-dependent (though with rather broad maxima) single-particle spectral function behavior, pretty far from the Fermi level, despite the fact that st these energies one can not speak about well defined quasiparticles. In this case we understand as particle dispersion precisely this $\mathbf{k}$-dependence of spectral function maxima which replaces the usual notion of quasiparticle spectrum. 


\subsubsection{Kinks of purely electronic nature}

Let us consider in more details the new mechanism of kinks formation in electronic dispersion of strongly correlated systems, which does not assume an interaction of electrons with phonons or other excitations [90]. In order to understand the nature of this mechanism at the beginning we shall examine weakly correlated system described by standard Fermi liquid theory. Because of large enough quasiparticles lifetime close to the Fermi level and weakness of correlations, in the first approximation interaction leads to a simple renormalization of initial dispersion of (noninteracting) quasiparticles $\epsilon_{\mathbf{k}}$, which is replaced by $E_{\mathbf{k}}=Z_{\mathrm{FL}} \epsilon_{\mathbf{k}}$, where $Z_{\mathrm{FL}}$ is electron Fermi liquid mass renormalization coefficient. It changes the slope of dispersion in the vicinity of the Fermi level. compared to a bare one. However, if we consider an electron with energy far away from the Fermi level, then in case of weak interaction one can expect that its dispersion practically does not change: $E_{\mathbf{k}} \approx \epsilon_{\mathbf{k}}$, though the damping here can be pretty large. In this sense one can say that electron-electron interaction by it self can result in formation of bends (kinks) in the generalized dispersion (of the spectral function), and position of such kink on the energy scale is defined right as an energy, where simple Fermi liquid picture becomes inapplicable. However, in weakly correlated metals $Z_{\mathrm{FL}} \lesssim 1$, so that the slope of $E_{\mathbf{k}}$ will be changed insignificantly, making such kinks hard to observe.

This picture can be essentially different in strongly correlated systems, where the value of $Z_{\mathrm{FL}}$ can be much less than one, thus making kinks more pronounced. Strong correlation interactions give rise to strong spectral weight redistribution within the singleparticle spectral function due to formation of Hubbard subbands. Moreover, as we know in strongly correlated metals there is also quasiparticle peak which appears close to the Fermi level, between Hubbard subbands. Below it will be shown that "usual" Fermi liquid quasiparticles exist in such systems only in a very narrow vicinity of the Fermi level. while beyond the Fermi liquid regime (but still "inside" of quasiparticle peak) exists some intermediate regime with dispersion $E_{\mathbf{k}} \approx Z_{\mathrm{CP}} \epsilon_{\mathbf{k}}$, where renormalization factor $Z_{\mathrm{CP}}$ is defined by central peak spectral weight and its value essentially differs from $Z_{\mathrm{FL}}$. At these intermediate energies, which are much smaller than interaction energy, electrons or holes are strongly interacting and their dispersion differs from both Fermi liquid one and noninteracting one. In this energy range one can speak about "intermediately" correlated situation, when $Z_{\mathrm{FL}}<Z_{\mathrm{CP}}<1$. Consequently, at some energies $\pm \omega_{\star}$ inside the quasiparticle peak transition from renormalization $Z_{\mathrm{FL}}$ to $Z_{\mathrm{CP}}$ takes place. That leads to an emergence of kink in electronic dispersion and the energy position of this kink is directly related to limits of applicability of the usual Fermi liquid theory. Lets emphasize that this mechanism gives rise to kink formation without any additional interaction with phonons or other collective modes. The only necessary condition for such kinks formation is the presence of strong electron-electron correlations in the system.

For microscopic description of electronic kinks lets consider Hubbard model, which will be analyzed in the framework of standard DMFT, using numerical renormalization group (NRG) to solve effective Anderson impurity problem. For simplicity we consider single-band Hubbard model at half-filling. Strongly correlated regime in Hubbard model occurs when interaction value becomes of the order of bare bandwidth $U \approx W$. Consider as an example computational results shown in Fig. 14. It is clearly visible that dispersion undergoes from Fermi liquid regime (line 1 on Fig. 14) into described above "intermediate" regime (line 2 in Fig. 14) with formation of well defined cusps in dispersion 


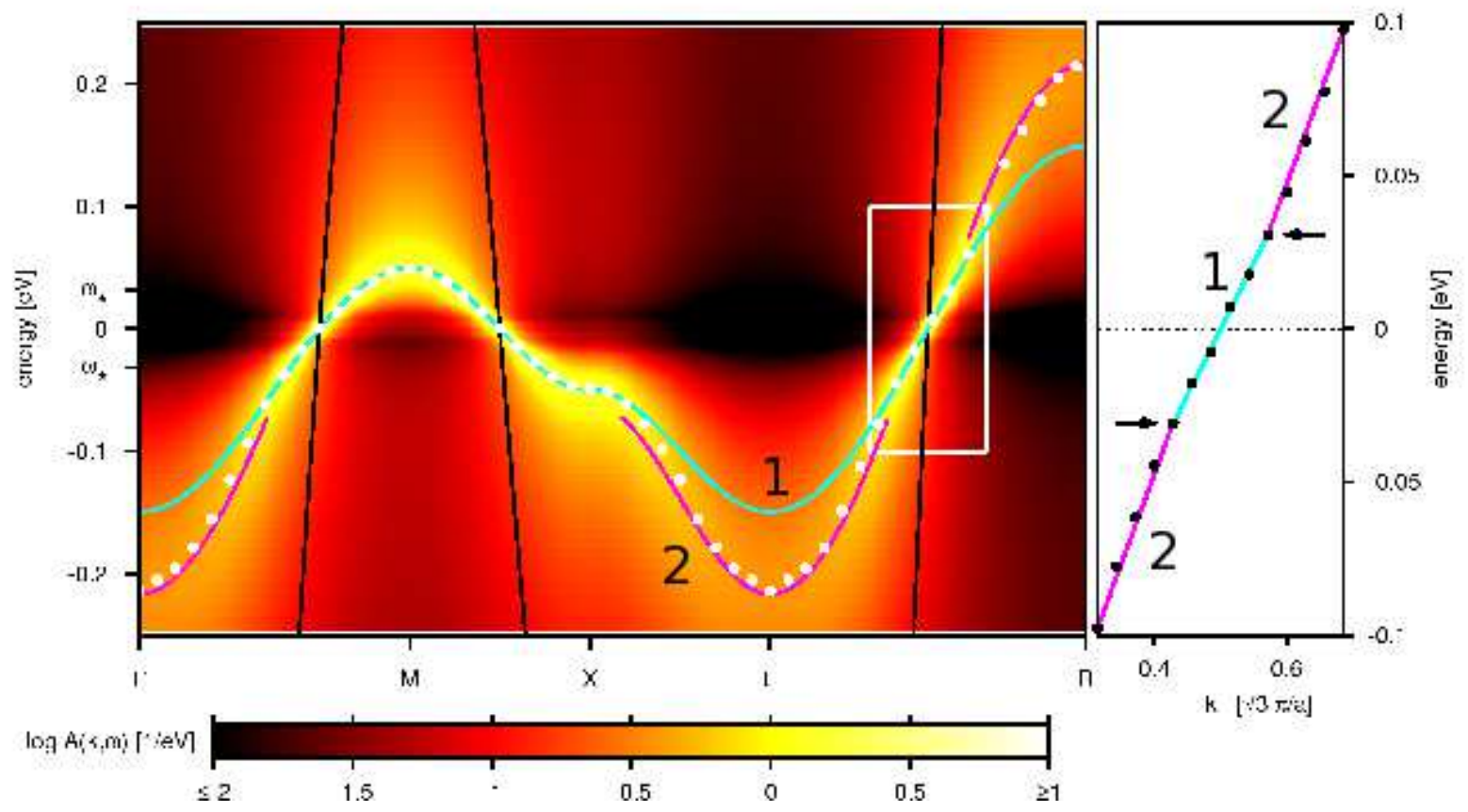

Figure 14: Cusps (kinks) in electronic dispersion $E_{\mathrm{k}}$ for the case of strongly correlated systems. On the contour plot intensity map of DMFT spectral function $A(\mathbf{k}, \omega)$ for the Hubbard model on a cubic lattice is presented. Hubbard interaction value is $U=3.5 \mathrm{eV}$, bandwidth $W \approx 3.64 \mathrm{eV}$, band filling $n=1$, calculated value of Fermi liquid renormalization is $Z_{\mathrm{FL}}=0.086$, temperature is $T=5 \mathrm{~K}$. Close to Fermi level maxima of $A(\mathbf{k}, \omega)$ (white dots) correspond to renormalized dispersion $E_{\mathbf{k}}=Z_{\mathrm{FL}} \epsilon_{\mathbf{k}}$ (line 1$)$. At energies $|\omega|>\omega_{\star}$ spectral function $A(\mathbf{k}, \omega)$ keeps its shape but with different renormalization $E_{\mathbf{k}}=Z_{\mathrm{CP}} \epsilon_{\mathbf{k}}-c \operatorname{sgn}\left(E_{\mathbf{k}}\right)$ (line 2). Values of $\omega_{\star}=0.03 \mathrm{eV}, Z_{\mathrm{CP}}=0.135$, and $c=0.018 \mathrm{eV}$ are calculated in [90] from the values of $Z_{\mathrm{FL}}$ and $\epsilon_{\mathrm{k}}$ corresponding to black line. Inset on the right shows in details part of dispersion in the $\Gamma$-R direction marked out with white rectangle, kinks (cusps in dispersion) at energies $\pm \omega_{\star}$ are pointed by arrows. Black lines - bare (noninteracting) quasiparticle spectra.

(kinks) at energies $\omega_{\star}=0.03 \mathrm{eV}$. In some of the high symmetry directions (e.g. around $\mathrm{X}$-point, Fig. 14) dispersion has quite small slope close to the Fermi level making kinks less pronounced.

Let us have a look on this situation from the point of view of single-particle Green's function behavior. From general considerations it is clear that any cusps of dispersion caused by interaction appear because of corresponding behavior of the self-energy or, more precisely, that of $\operatorname{Re} \Sigma_{\mathbf{k}}(\omega)$. In the majority of real physical systems $\mathbf{k}$-dependence of self-energy, apparently, is less important in comparison with $\omega$-dependence and thus the neglect of self-energy k-dependence $\Sigma_{\mathbf{k}}(\omega)=\Sigma(\omega)$ is, more or less, good approximation. In the framework of DMFT this statement is exact. Self-consistent expression for selfenergy of Hubbard model within DMFT can be written as:

$$
\Sigma(\omega)=\omega+\mu-1 / G(\omega)-\Delta(G(\omega)),
$$

where $G(\omega)=\frac{1}{N} \sum_{\mathbf{k}} G(\mathbf{k}, \omega)$ is the local (averaged over $\mathbf{k}$ ) Green's function, $\Delta(G)$ is 
frequency dependent hybridization function expressed via $G(\omega) 6$. Hybridization function describes quantum-mechanical coupling between an electron on a given site and other sites of the system.

In Fig. [15] we show frequency dependence of spectral function (DOS) $A(\omega)=-\operatorname{Im} G(\omega) / \pi$ calculated for the same model parameters as in Fig. 14 and demonstrating a typical threepeak structure. Corresponding real parts of Green's function $G(\omega)$ and self-energy $\Sigma(\omega)$ are shown in Fig. 15b and Fig. 15k.

Kinks in $\operatorname{Re} \Sigma(\omega)$ are directly related to three-peak structure of integrated spectral function (DOS) $A(\omega)$ (its minima are at energies $\pm \Omega$ ) and define some new quite small energy scale. To this end $\operatorname{Re}[G(\omega)]$ should have maxima and minima in the energy interval $\pm \omega_{\max }$, i.e. "inside" the central peak (Fig. 15b). In its turn it directly leads to kinks formation in $\operatorname{Re} \Sigma(\omega)$. Self-energy $\Sigma(\omega)$ consists of two contributions: $\omega+\mu-1 / G(\omega)$ and $-\Delta(G(\omega))$. Expression $\operatorname{Re}[\omega+\mu-1 / G(\omega)]$ is linear within quite large energy range $|\omega|<\Omega$ (Fig. [15d), while the value of $-\operatorname{Re}[\Delta(G(\omega))]$ is proportional to $-\operatorname{Re}[G(\omega)]$, at least in the first order of corresponding momenta expansion, only in the small energy interval $|\omega|<\omega_{\max }$. The sum of these two contributions gives rise to cusps in real part of self-energy at energies $\pm \omega_{\star}$, where $\omega_{\star}=(\sqrt{2}-1) \omega_{\max }$. At this energy $\operatorname{Re}[G(\omega)]$ has maximal curvature (these points are marked out by circles in Fig. 15r). Thus the Fermi liquid regime, when the slope of the real part of self-energy is described by $\partial \operatorname{Re} \Sigma(\omega) / \partial \omega=$ $1-1 / Z_{\mathrm{FL}}$, exists only within a narrow part of the central peak, namely in the energy interval $|\omega|<\omega_{\star}$. At higher (intermediate) energies the slope of $\operatorname{Re} \Sigma(\omega)$ will be given by $\partial \operatorname{Re} \Sigma(\omega) / \partial \omega=1-1 / Z_{\mathrm{CP}}$. As a result, effective dispersion $E_{\mathbf{k}}$ will manifest kinks at energies $\omega_{\star}$.

This analysis also helps to understand why outside Fermi liquid region $E_{\mathbf{k}}$ demonstrates another value of renormalization of noninteracting electron dispersion, given by $Z_{\mathrm{CP}}$ with small offset $c$. This behavior is determined by the value of main contribution to self-energy $\omega+\mu-1 / G(\omega)$ at energies $\omega_{\star}<|\omega|<\Omega$ i.e. within the central peak of DOS. Values of $\omega_{\star}, Z_{\mathrm{CP}}$, and $c$ can be expressed via $Z_{\mathrm{FL}}$ together with characteristics of noninteracting electron density of states. One can show that $\omega_{\star}=Z_{\mathrm{FL}}(\sqrt{2}-1) D$, where $D$ is the halfwidth of the bare band (details see in work 90]). If correlations are weak and $Z_{\mathrm{FL}} \lesssim 1$ kinks positions in $E_{\mathbf{k}}$ practically coincide with the edges of bare electron band, which makes them almost unobservable. On the other hand, in the strongly correlated regime $\left(Z_{\mathrm{FL}} \ll 1\right)$ kinks energy $\omega_{\star} / D \propto Z_{\mathrm{FL}}$ approaches the Fermi level inside the central peak, which width diminishes with the increase of correlations as $\Omega / D \propto \sqrt{Z_{\mathrm{FL}}}$ [94].

For the first time, these purely electronic kinks were observed in LDA+DMFT calculations for $\mathrm{SrVO}_{3}$ system [92]. Definition of energy scale $\omega_{\star}$ contains only parameters of initial band structure, which can be obtained (for realistic systems) via band structure calculations, together with Fermi liquid mass renormalization $Z_{\mathrm{FL}}=1 /(1-\partial \operatorname{Re} \Sigma(0) / \partial \omega) \equiv$ $\mathrm{m} / \mathrm{m}^{*}$, which can be experimentally determined from specific heat or spin susceptibility measurements In particular, in Ref. [93] it was shown that kinks of electronic nature can cause corresponding cusps in the linear (in temperature) term of specific heat of strongly correlated metals, which was, apparently, observed in $\mathrm{LiV}_{2} \mathrm{O}_{4}$ heavy fermion system.

\footnotetext{
${ }^{6}$ In DMFT $\Delta(G)$ is defined by $G(\omega)=G_{0}(\omega+\mu-\Sigma(\omega))$, i.e. $G_{0}(\Delta(G)+1 / G)=G$, where $G_{0}(\omega)$ is noninteracting local Green's function.
} 

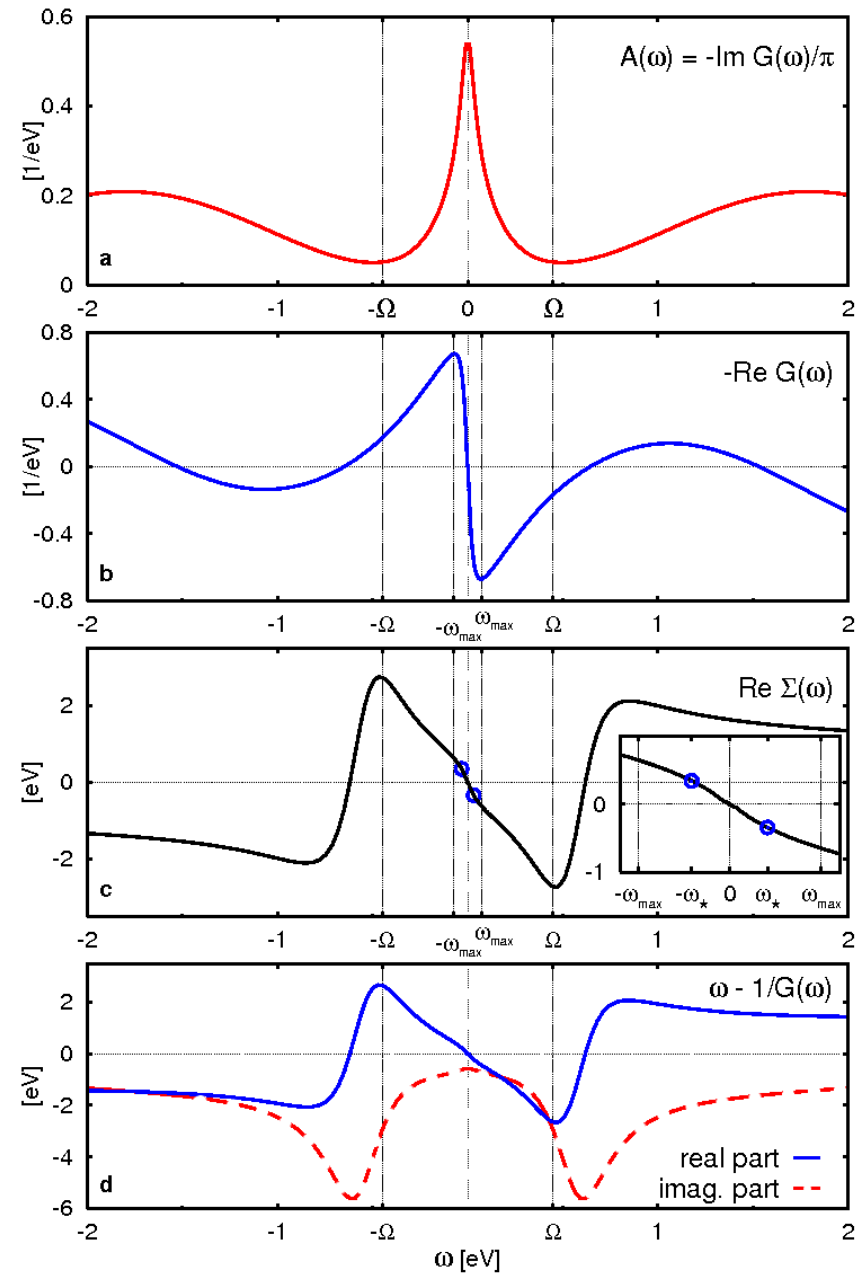

Figure 15: Green's function characteristic for strongly correlated system with parameters given in the caption of Fig. 14, a - three-peak structure of spectral function $A(\omega)=$ $-\operatorname{Im} G(\omega) / \pi$ with minima at energies $\pm \Omega=0.45 \mathrm{eV}$. b - real part of Green's function with relative minima and maxima at energies $\pm \omega_{\max }$ located "inside" quasiparticle peak. c - real part of self-energy with cusps at energies $\pm \omega_{\star}$ (circles) situated in the points of maximal curvature of $\operatorname{Re} G(\omega)\left(\omega_{\star}=0.4 \omega_{\max }=0.03 \mathrm{eV}\right) . \mathbf{d}-$ real part of $\omega-1 / G(\omega)$ (solid line) contributing to self-energy which is linear, in general, in the energy interval $|\omega|<\Omega$. Change of energy dependence of $-\operatorname{Re}[G(\omega)]$ at energies $\pm \omega_{\star}$ defines the location of kinks (cusps) in electronic dispersion. 


\subsubsection{Role of electron-phonon interaction}

The previous section material inevitably brings us to the question about the relation and mutual influence of kinks of electronic nature and the "usual" kinks in the electron dispersion induced by electron-phonon interaction. This is related to a more general problem of joint influence between strong electronic correlations and electron-phonon interaction. In fact, the history of such investigations is relatively long and one of the most popular models of electron-phonon interaction in strongly correlated systems is Hubbard-Holstein model. Hubbard model describes local Coulomb interaction on a lattice. On the other hand, Holstein model describes linear interaction of conduction electrons with local (Einstein) phonon modes [95. Studies of Hubbard-Holstein model were performed in the framework of conventional DMFT [5], in particular with the use of numerical renormalization group (NRG) [15] as "impurity solver". Reducing of Hubbard-Holstein model to Anderson-Holstein impurity problem was first performed by Hewson and Mayer [96]. They showed that using NRG one can calculate the total electron-phonon contribution to self-energy, thus achieving nonperturbative solution of Hubbard-Holstein model not only with respect to Hubbard interaction, but also with respect to electron-phonon interaction. Let us note that the general structure of DMFT equations in this approach is preserved.

However, until recently there were no studies of strongly correlated electrons interacting with Debye phonons. It is quite surprising in view of rather wide discussion of the physics of kinks in electron dispersion observed in ARPES experiments in high- $\mathrm{T}_{c}$ superconducting oxides [80]. The origin of these kinks is typically attributed to electron-phonon interaction [81]. Problem of kinks formation in electron dispersion caused by electronphonon interaction in strongly correlated systems was shortly discussed in the framework of Hubbard-Holstein model Ref. [97, 98]. In this section we overview DMFT $+\Sigma$ results for Hubbard model with added interaction with Debye phonons, under the assumption of the validity of Migdal theorem (adiabatic approximation). This approximation is reasonable for electron-phonon coupling constant $\lambda<E_{F} / \omega_{D} \sim 10$, where $E_{F}$ is Fermi energy, $\omega_{D}$ is Debye frequency.

To consider electron-phonon interaction in the strongly correlated system we introduce self-energy $\Sigma_{\mathbf{k}}(\varepsilon)=\Sigma_{p h}(\varepsilon, \mathbf{k})$, appearing in the usual Froehlich model. According to Migdal theorem, in adiabatic approximation we can restrict ourselves to the simplest first order contribution to $\Sigma_{p h}(\varepsilon, \mathbf{k})$. Main advantage of adiabatic approximation is the possibility to neglect any vertex corrections from electron-phonon interaction, which are small over adiabatic parameter $\frac{\omega_{D}}{E_{F}} \ll 1[99]$.

In fact, $\Sigma_{p h}(\varepsilon, \mathbf{k})$ in this approximation has only weak momentum dependence, which can be neglected and we have to account only for essential frequency dependence. Direct calculations (see e.g. [29]) in the case of Debye phonon spectra $\omega_{0}(\mathbf{k})=u|\mathbf{k}|$ for $|\mathbf{k}|<\frac{\omega_{D}}{u}$, where $u$ is sound velocity, give:

$$
\Sigma_{p h}(\varepsilon)=\frac{-i g^{2}}{4 \omega_{c}^{2}} \int_{-\infty}^{+\infty} \frac{d \omega}{2 \pi}\left\{\omega_{D}^{2}+\omega^{2} \ln \left|\frac{\omega_{D}^{2}-\omega^{2}}{\omega^{2}}\right|+i \pi \omega^{2} \theta\left(\omega_{D}^{2}-\omega^{2}\right)\right\} I(\varepsilon+\omega),
$$

where $g$ is the usual electron-phonon interaction constant $I(\epsilon)=\int_{-D}^{+D} d \xi \frac{N_{0}(\xi)}{E_{\varepsilon}-\xi}, E_{\varepsilon}=$ $\varepsilon-\Sigma(\varepsilon)-\Sigma_{p h}(\varepsilon)$ and $\omega_{c}=p_{F} u$ is characteristic frequency of the order of Debye one. In case of semielliptic bare DOS $N_{0}(\varepsilon)$ with halfwidth $D$ one obtains $I(\epsilon)=\frac{2}{D^{2}}\left(E_{\varepsilon}-\sqrt{E_{\varepsilon}^{2}-D^{2}}\right)$. It is convenient to introduce dimensionless electron-phonon coupling constant, which for 

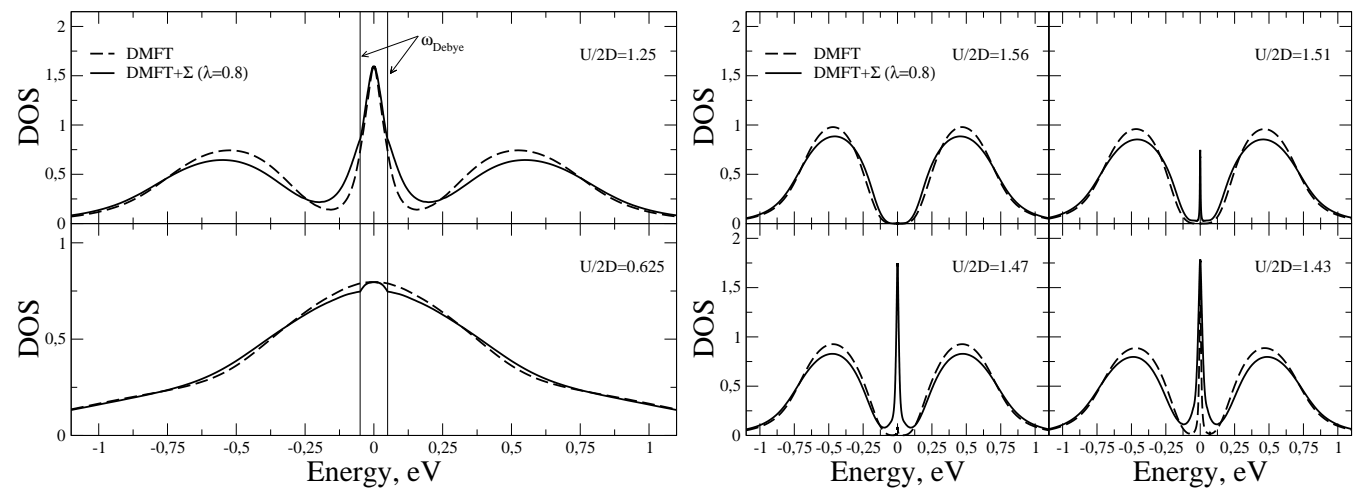

Figure 16: Comparison of DMFT (dashed line) and DMFT $+\Sigma_{p h}$ (solid line) densities of states. On the left side - for strong (upper panel, $U / 2 D=1.25$ ) and weak (lower panel, $U / 2 D=0.625$ ) Hubbard interactions. On the right side - densities of states evolution close to metal-insulator transition. Dimensionless electron-phonon coupling constant is $\lambda=0.8$ [100, 101].

this model can be written as [29]:

$$
\lambda=g^{2} N_{0}\left(\varepsilon_{F}\right) \frac{\omega_{D}^{2}}{4 \omega_{c}^{2}}
$$

To simplify calculations we neglect renormalization of phonons because of electron-phonon interaction [29], assuming in the following that phonon spectrum is fixed by experiment.

\subsubsection{Electronic and phonon kinks within the DMFT $+\Sigma$ approach}

Let us focus on most interesting $\mathrm{DMFT}+\Sigma$ results in this model, referring the reader for details to Refs. [100, 101]. Here we present results obtained for the case of interaction of electrons with Debye phonons (results for Einstein phonons is analogous [101]).

Comparison of DMFT and DMFT $+\Sigma_{p h}$ densities of states with electron-phonon interaction for strong $(\mathrm{U} / 2 \mathrm{D}=1.25)$ and weak $(\mathrm{U} / 2 \mathrm{D}=0.625)$ Hubbard interaction is presented on upper and lower panels on the left side of Fig. 16. Dimensionless constant (46) exploited in these calculations was chosen to be $\lambda=0.8$ and Debye frequency $\omega_{D}=0.125 \mathrm{D}$. In both cases we observe some spectral weigh transfer caused by electron-phonon interaction. For $\mathrm{U} / 2 \mathrm{D}=1.25$ (upper panel of Fig. 16) we see well developed three-peak structure typical for strongly correlated systems. In the energy interval $\pm \omega_{D}$ around the Fermi level (which is zero energy in all figures) practically there is no difference in the shape of quasiparticle peak in the DOS obtained within DMFT and DMFT $+\Sigma_{p h}$. But beyond this interval DMFT $+\Sigma_{p h}$ quasiparticle peak becomes significantly wider because of partial transfer of spectral weight from Hubbard bands. This broadening of quasiparticle peak in $\mathrm{DMFT}+\Sigma_{p h}$ leads to a delay of metal-insulator transition as we shall see later.

In case of $\mathrm{U} / 2 \mathrm{D}=0.625$ no clearly distinguishable Hubbard bands are formed and we observe only some side wings in the DOS. Redistribution of spectral weight on the lower panel of Fig. 16 is not very dramatic despite qualitative difference with the $\mathrm{U} / 2 \mathrm{D}=1.25$ case. Main distinction between DMFT and DMFT $+\Sigma_{p h}$ results occurs here in the interval $\pm \omega_{D}$, where formation of a "cap" in DOS is observed, due to electron-phonon interaction. Corresponding spectral weight goes to the energies around $\pm U$ where Hubbard bands start to form. 
On the right panel of Fig.. 16 we compare behavior of densities of states in DMFT without phonons and in $\mathrm{DMFT}+\Sigma_{p h}$, for different values of $\mathrm{U} / 2 \mathrm{D}$ parameter nearby Mott-Hubbard transition. At $\mathrm{U} / 2 \mathrm{D}=1.56$ both standard DMFT and DMFT $+\Sigma_{p h}$ give insulating solution. However, there are some distinctions between these solutions. In DMFT $+\Sigma_{p h}$ Hubbard bands are lower and wider than in DMFT since additional (electronphonon) interaction is included. With decrease of $\mathrm{U}$ at $\mathrm{U} / 2 \mathrm{D}=1.51$ and 1.47 we observe that DMFT $+\Sigma_{p h}$ results correspond to metallic state (with narrow quasiparticle peak on the Fermi level), while DMFT without phonons still shows insulating solution. Only about $\mathrm{U} / 2 \mathrm{D}=1.43$ both DMFT and DMFT $+\Sigma_{p h}$ results for DOS correspond to the metallic state. Thus, under increase of $U$ the finite value of electron-phonon interaction slightly delays Mott-Hubbard transition from metallic to insulating phase. This result is analogous to one obtained within the Hubbard-Holstein model for weak electron-phonon coupling [102, 103, 104.

Therefore, moderate electron-phonon interaction brings to rather insignificant changes of electron density of states both in correlated metal and in Mott insulating state, only slightly delaying transition from metal to insulator with the growth of $U$.

Now we turn to the source of sharp slope changes of electron dispersion (kinks). It is well known that in general case kinks are formed because of interaction of electrons with bosonic modes. In case of electron-phonon interaction, typical energy of the kink is about Debye (or Einstein) frequency. Above we have shown that in strongly correlated metal kinks of purely electronic nature can arise [90]. Energy of such kink for semielliptical bare DOS is $\omega^{*}=Z_{F L}(\sqrt{2}-1) D$, where D is the halfwidth of the "bare" band and $Z_{F L}=\left(1-\left.\frac{\partial R e \Sigma)}{\partial \varepsilon}\right|_{\varepsilon=E_{F}}\right)^{-1}$ is Fermi liquid renormalization factor. Roughly speaking $\omega^{*}$ is defined by halfwidth of the quasiparticle peak in DOS.

Kink of electronic nature is quite smooth and its observation is rather difficult. DMFT $+\Sigma_{p h}$ calculations show that electronic kinks are hardly detectable on the background of phonon kinks and fine "tuning" of model parameters is necessary pick them out. First of all, it is necessary to guarantee that $\omega_{D} \ll \omega^{*}$ (in other cases smooth electronic kinks will be practically indiscernible against kinks from electron-phonon interaction). For $\mathrm{U} / 2 \mathrm{D}=1$ and $\mathrm{U}=3.5 \mathrm{eV}$ we have $\omega^{*} \sim 0.1 \mathrm{D}$, while Debye frequency can be taken quite small e.g. $\omega_{D} \sim 0.01 \mathrm{D}$. In order to make phonon kink pronounced enough at such relatively low Debye frequency one needs to increase electron-phonon coupling constant up to $\lambda=2.0$.

To demonstrate the possibility of coexistence of both types of kinks in the spectra, let us consider the energy dispersion for simple cubic lattice with nearest neighbors hopping only. Most convenient is to discuss the high symmetry direction $\Gamma-(\pi, \pi, \pi)$ of the Brillouin zone [90]. On the left panel of Fig. 17 we show electronic dispersion along this direction close to the Fermi level. Line with diamonds is the electron spectrum of standard DMFT without phonons. Lines with circles presents DFMT $+\Sigma_{p h}$ results. Electronic and phonon kinks are marked by arrows. In general case, kinks from electronphonon interaction dominate for most typical model parameters making electronic kink observation predicted in [90] quite difficult.

In conclusion we give the picture of phonon kinks evolution depending on the value of Hubbard interaction $U$. With decrease of $U / 2 D$ ratio Fermi velocity goes down and kink position on the momentum axis shifts farther away from $p_{F}$, while the kink energy remains about $\omega_{D}$. This behavior follows from direct DMFT $+\Sigma_{p h}$ calculations [100, 101] and is shown on the right panel of Fig. 17. In the case of interaction with Einstein phonons results are quite analogous [101. 

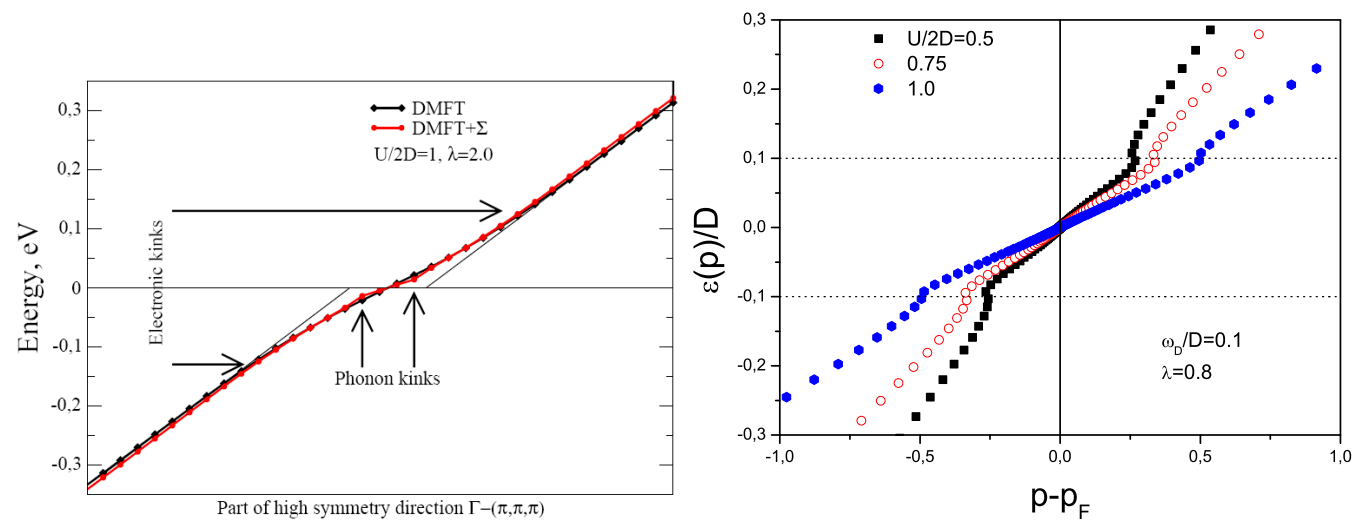

Figure 17: Quasiparticle dispersion with kinks around the Fermi level. On the left side dispersion along $\Gamma-(\pi, \pi, \pi)$ high symmetry direction in the Brillouin zone for the case of simple cubic lattice and bare dispersion with nearest neighbors hopping only: DMFT (curve with diamonds) and DMFT $+\Sigma_{p h}$ (curve with circles) $\left(\lambda=2, \omega_{D}=0.01 D\right)$. On the right side - DMFT $+\Sigma_{p h}$ phonon kinks evolution for different values of Hubbard interaction $U / 2 D=0.5$, $0.75,1.0 ; \lambda=0.8, \omega_{D}=0.1 D$ [100].

\section{Electronic structure of real strongly correlated sys- tems: LDA+DMFT and LDA+DMFT $+\Sigma$}

At present the most advanced ab initio (i.e. ideally without any fitting parameters) computational scheme of electron spectra of realistic strongly correlated systems is LDA+DMFT method [2, 16]. LDA band structure in this method is used to obtain "noninteracting" starting Hamiltonian, while strong correlations are accounted afterwards within DMFT. In fact, LDA+DMFT computational scheme combines two scientific areas: "realistic" band structure calculations and traditional model approaches, which were essentially separated from each other before. Without DMFT just in LDA there is no way to describe strongly correlated systems, while without LDA calculations manybody methods can not be material specific. Below we briefly discuss the basics of LDA+DMFT and generalized $\mathrm{LDA}+\mathrm{DMFT}+\Sigma$ methods.

\subsection{Density functional theory (DFT). Local density approxima- tion (LDA).}

In Born-Oppenheimer adiabatic approximation [105], neglecting relativistic effects, electronic properties in solid state physics are described by the Hamiltonian:

$$
\begin{aligned}
\hat{H}= & \sum_{\sigma} \int d^{3} r \hat{\Psi}^{+}(\mathbf{r}, \sigma)\left[-\frac{\hbar^{2}}{2 m_{e}} \Delta+V_{\mathrm{ion}}(\mathbf{r})\right] \hat{\Psi}(\mathbf{r}, \sigma) \\
& +\frac{1}{2} \sum_{\sigma \sigma^{\prime}} \int d^{3} r d^{3} r^{\prime} \hat{\Psi}^{+}(\mathbf{r}, \sigma) \hat{\Psi}^{+}\left(\mathbf{r}^{\prime}, \sigma^{\prime}\right) V_{\mathrm{ee}}\left(\mathbf{r}-\mathbf{r}^{\prime}\right) \hat{\Psi}\left(\mathbf{r}^{\prime}, \sigma^{\prime}\right) \hat{\Psi}(\mathbf{r}, \sigma) .
\end{aligned}
$$


Here $\hat{\Psi}^{+}(\mathbf{r}, \sigma)$ and $\hat{\Psi}(\mathbf{r}, \sigma)$ are creation and annihilation operators of electron with coordinate $\mathbf{r}$ and spin $\sigma, \Delta$ is the Laplace operator, $m_{e}$ is electron mass, $e$ is electron charge,

$$
V_{\text {ion }}(\mathbf{r})=-e^{2} \sum_{i} \frac{Z_{i}}{\left|\mathbf{r}-\mathbf{R}_{i}\right|} \quad, \quad V_{\mathrm{ee}}\left(\mathbf{r}-\mathbf{r}^{\prime}\right)=\frac{e^{2}}{2} \sum_{\mathbf{r} \neq \mathbf{r}^{\prime}} \frac{1}{\left|\mathbf{r}-\mathbf{r}^{\prime}\right|}
$$

denote single particle potential created by all ions $i$ with charge $e Z_{i}$ located at given positions $\mathbf{R}_{i}$ and electron-electron interaction.

Although the "first principle" Hamiltonian (47) is easy to write down, it is impossible to solve corresponding quantum mechanical problem exactly. This is the reason to make substantial physical approximations. In particular, density functional theory (DFT) is based on Hohenberg-Kohn theorem [106] (see also the review [107]), which asserts that ground state energy is the unique functional of electron charge density, which is minimal for the equilibrium electron density in the ground state:

$$
E[\rho]=E_{\text {kin }}[\rho]+E_{\text {ion }}[\rho]+E_{\text {Hartree }}[\rho]+E_{\text {xc }}[\rho],
$$

where the Hartree energy $E_{\text {Hartree }}[\rho]=\frac{1}{2} \int d^{3} r^{\prime} d^{3} r \quad V_{\text {ee }}\left(\mathbf{r}-\mathbf{r}^{\prime}\right) \rho\left(\mathbf{r}^{\prime}\right) \rho(\mathbf{r})$ and potential energy of ions $E_{\text {ion }}[\rho]=\int d^{3} r V_{\text {ion }}(\mathbf{r}) \rho(\mathbf{r})$, can be directly expressed via electron charge density. The term $E_{\text {kin }}[\rho]$ denotes the kinetic energy of electrons and $E_{\text {xc }}[\rho]$ is unknown, in general, exchange-correlation term, containing electron-electron interaction energy beyond the Hartree term. In fact, all peculiarities of the manybody problem are transferred into the computation of $E_{\mathrm{xc}}[\rho]$.

In practice, instead of minimization of $E[\rho]$ over $\rho$, minimization is performed usually over some set of orthonormal functions $\varphi_{i}$, related to $\rho$ via expression:

$$
\rho(\mathbf{r})=\sum_{i=1}^{N}\left|\varphi_{i}(\mathbf{r})\right|^{2} .
$$

Introducing arbitrary Lagrange parameters $\varepsilon_{i}$ and requiring

$$
\frac{\delta}{\delta \varphi_{i}(\mathbf{r})}\left\{E[\rho]+\varepsilon_{i}\left[1-\int d^{3} r\left|\varphi_{i}(\mathbf{r})\right|^{2}\right]\right\}=0
$$

one gets Kohn-Shem equations [108]:

$$
\left[-\frac{\hbar^{2}}{2 m_{e}} \Delta+V_{\mathrm{ion}}(\mathbf{r})+\int d^{3} r^{\prime} V_{\mathrm{ee}}\left(\mathbf{r}-\mathbf{r}^{\prime}\right) \rho\left(\mathbf{r}^{\prime}\right)+\frac{\delta E_{\mathrm{xc}}[\rho]}{\delta \rho(\mathbf{r})}\right] \varphi_{i}(\mathbf{r})=\varepsilon_{i} \varphi_{i}(\mathbf{r}) .
$$

Formally this equation coincides with single particle stationary Schroedinger equation. Electron kinetic energy corresponding to charge density of the ground state is given now by the expression

$$
E_{\text {kin }}\left[\rho_{\min }\right]=-\sum_{i=1}^{N}\left\langle\varphi_{i}\left|\hbar^{2} \Delta /\left(2 m_{e}\right)\right| \varphi_{i}\right\rangle,
$$

where $\varphi_{i}$ are self-consistent (spin degenerate) solutions of equations (52) and (501), corresponding to the smallest single particle energy $\epsilon_{i}$ [109].

Most common way to calculate $E_{\mathrm{xc}}[\rho]$ is to use the local density approximation (LDA). It approximates the functional $E_{\mathrm{xc}}[\rho]$ by the local charge density functional:

$$
E_{\mathrm{xc}}[\rho] \rightarrow \int d^{3} r \rho(\mathbf{r}) \epsilon_{\mathrm{xc}}^{\mathrm{LDA}}(\rho(\mathbf{r}))
$$


Explicit expression for $\epsilon_{\mathrm{xc}}^{\mathrm{LDA}}(\rho(\mathbf{r}))$ can be found in the framework of different models e.g. from numerical analysis of "jellium" model (electronic gas on a positive ionic background) [110]. by

In fact, LDA approximation corresponds to the replacement of the Hamiltonian (47)

$$
\begin{aligned}
\hat{H}_{\mathrm{LDA}}=\sum_{\sigma} \int d^{3} r \hat{\Psi}^{+}(\mathbf{r}, \sigma)[ & -\frac{\hbar^{2}}{2 m_{e}} \Delta+V_{\mathrm{ion}}(\mathbf{r})+\int d^{3} r^{\prime} \rho\left(\mathbf{r}^{\prime}\right) V_{\mathrm{ee}}\left(\mathbf{r}-\mathbf{r}^{\prime}\right) \\
& \left.+\frac{\delta E_{\mathrm{xc}}^{\mathrm{LDA}}[\rho]}{\delta \rho(\mathbf{r})}\right] \hat{\Psi}(\mathbf{r}, \sigma) .
\end{aligned}
$$

In practical calculations electron field operators are expanded over some set of atomiclike functions (basis set) $\Phi_{i l m}$ ( $i$ denotes lattice site, $l$ is orbital quantum number, $m$ is magnetic quantum number). In this representation

$$
\hat{\Psi}^{+}(\mathbf{r}, \sigma)=\sum_{i l m} \hat{c}_{i l m}^{\sigma \dagger} \Phi_{i l m}(\mathbf{r})
$$

and the Hamiltonian (55) is rewritten as

$$
\hat{H}_{\mathrm{LDA}}=\sum_{i l m, j l^{\prime} m^{\prime}, \sigma}\left(\delta_{i l m, j l^{\prime} m^{\prime}} \varepsilon_{i l m} \hat{n}_{i l m}^{\sigma}+t_{i l m, j l^{\prime} m^{\prime}} \hat{c}_{i l m}^{\sigma \dagger} \hat{c}_{j l^{\prime} m^{\prime}}^{\sigma}\right)
$$

Here $\hat{n}_{i l m}^{\sigma}=\hat{c}_{i l m}^{\sigma \dagger} \hat{c}_{i l m}^{\sigma}$ is electron density operator on a given orbital, while matrix elements:

$$
t_{i l m, j l^{\prime} m^{\prime}}=\left\langle\Phi_{i l m}\left|-\frac{\hbar^{2} \Delta}{2 m_{e}}+V_{\mathrm{ion}}(\mathbf{r})+\int d^{3} r^{\prime} \rho\left(\mathbf{r}^{\prime}\right) V_{\mathrm{ee}}\left(\mathbf{r}-\mathbf{r}^{\prime}\right)+\frac{\delta E_{\mathrm{xc}}^{\mathrm{LDA}}[\rho]}{\delta \rho(\mathbf{r})}\right| \Phi_{j l^{\prime} m^{\prime}}\right\rangle
$$

in case of $i l m \neq j l^{\prime} m^{\prime}$ define effective hopping integrals and single particle energies $\varepsilon_{i l m}$ are given by corresponding diagonal expressions in case of identical indices. On this step purely analytic work ends and numerical calculations follow within the chosen basis set, e.g. linearized muffin-tin orbitals (LMTO). Specific expressions for matrix elements (58) within the LMTO basis are presented in Ref. [111].

\subsection{LDA+DMFT computational scheme}

The primary importance for strongly correlated materials is the onsite Coulomb interaction between $d$ - or $f$-electrons, since this contribution to interaction energy is the largest one. The largest nonlocal contribution is density-density type interaction between nearest neighbors, where the main contribution comes from Hartree term (see [112] and [113]), which is already taken into account in the LDA. Moreover in LDA some part of exchangecorrelation interactions is taken into account via effective potential $\frac{\delta E_{\mathrm{xc}}^{\mathrm{LDA}}[\rho]}{\delta \rho(\mathbf{r})}$.

In order to take into account strong local Coulomb interaction one should supplement Hamiltonian (57) with approximate Coulomb matrix with most important parameters only [16]: $U$ - onsite intraband Coulomb repulsion, $J$ - exchange interaction and interorbital Coulomb repulsion $U^{\prime}$, acting on different electronic orbitals of the same site $i_{d}$, 
where sits an atom with partially filled $d$-shell $(l$-orbital quantum number, $m$ - magnetic quantum number, $\sigma-$ spin index):

$$
\begin{aligned}
\hat{H}=\hat{H}_{\mathrm{LDA}} & +U \sum_{m} \sum_{i=i_{d}, l=l_{d}} \hat{n}_{i l m \uparrow} \hat{n}_{i l m \downarrow} \\
& +\sum_{i=i_{d}, l=l_{d}} \sum_{m \neq m^{\prime}} \sum_{\sigma \sigma^{\prime}}\left(U^{\prime}-\delta_{\sigma \sigma^{\prime}} J\right) \hat{n}_{i l m \sigma} \hat{n}_{i l m^{\prime} \sigma^{\prime}} \\
& -\sum_{i=i_{d}, l=l_{d}} \sum_{m \sigma} \Delta \epsilon_{d} \hat{n}_{i l m \sigma}
\end{aligned}
$$

where for simplicity only density-density type interactions are left, and so called Kanamori parametrization is applied, when for the same orbitals $\left(m=m^{\prime}\right)$ direct Coulomb interaction is taken as $U$, while for different orbitals $\left(m \neq m^{\prime}\right)$ this interaction is equal to $U^{\prime}$. Because of rotational invariance of the single atom problem $U^{\prime}=U-2 J$ and exchange interaction parameter does not depend on orbital index and is equal to $J$.

Moreover, in (60) the last $\Delta \epsilon_{d}$ - term is added (the so called double counting correction), which should correct for double counting, as some part of local Coulomb interaction is already included into $\hat{H}_{\mathrm{LDA}}$. General expression for $\Delta \epsilon_{d}$ via $U$ and $\rho$ is unknown. However, there are several qualitative recipes to determine the value of $\Delta \epsilon_{d}$, and which are employed in different modern LDA+DMFT calculations (detailed discussion can be found in Refs. [114, 115]). The simplest physical assumption is that within the DFT Coulomb interaction energy can be written as:

$$
E_{D F T}=\frac{1}{2} \bar{U} n_{d}\left(n_{d}-1\right)
$$

where $n_{d}$ is the total number of electrons on $d$-shell and $\bar{U}$ is the average Coulomb interaction (here we assume averaging over all orbital pairs $m \sigma, m^{\prime} \sigma^{\prime}$ on a given site). Thus $\Delta \epsilon_{d}$ is taken as:

$$
\Delta \epsilon_{d}=\frac{\partial E_{D F T}}{\partial n_{d}}=\bar{U}\left(n_{d}-\frac{1}{2}\right) .
$$

The values of interaction parameters $U, J$ and $U^{\prime}$ can be obtained from the averaged Coulomb interaction $\bar{U}$ and Hund exchange parameter $J$. The averaged interaction $\bar{U}$ is related to $U$ and $U^{\prime}$ parameters via the following relation:

$$
\bar{U}=\frac{U+\left(N_{\text {orb }}-1\right) U^{\prime}+\left(N_{\text {orb }}-1\right)\left(U^{\prime}-J\right)}{2 N_{\text {orb }}-1},
$$

where $\mathrm{N}_{\text {orb }}$ is the number of interacting orbitals. Since $U$ and $U^{\prime}$ are not independent parameters $\bar{U}$ and $J$ are sufficient to determine $U$ [20, 116].

For microscopic calculations of averaged Coulomb interaction different methods were developed, such as "constrained LDA" [117] or "constrained RPA" [118, 119]. Generalizations to calculate Hund exchange parameter also exist. Unfortunately, there are rather large discrepancies between the values of parameters obtained with these methods. The value of $\bar{U}$ appears to be strongly dependent on the basis set used (e.g. in the problem of screening of long-range part of Coulomb interaction). It is clear that introduction of all these essentially model parameters takes us quite far away from "first principle" ideal, though it is the best one can do at the moment to calculate band structure of solids with 
transition metal atoms, where electron-electron interactions play the crucial role. In that sense it is probably more correct to speak about "modelling" of electron structure of such systems.

Matrix elements of "noninteracting" Hamiltonian in the reciprocal space $H_{\mathrm{LDA}}^{0}(\mathbf{k})$ can be calculated numerically at every point of the Brillouin zone, then the integrals over the Brillouin zone usually are calculated with tetrahedron method [120]. In case of relatively simple band dispersions, when analytical expression for $H_{\mathrm{LDA}}^{0}(\mathbf{k})$ dependence on $\mathbf{k}$ can be written explicitly, the values of hopping integrals can be found from LDA by projecting on corresponding Wannier functions [121, 122. Matrix elements of this Hamiltonian, i.e. single particle LDA energies without local Coulomb interaction can be written in a following way:

$$
\left(H_{\mathrm{LDA}}^{0}(\mathbf{k})\right)_{q l m, q^{\prime} l^{\prime} m^{\prime}}=\left(H_{\mathrm{LDA}}(\mathbf{k})\right)_{q l m, q^{\prime} l^{\prime} m^{\prime}}-\delta_{q l m, q^{\prime} l^{\prime} m^{\prime}} \delta_{q l, q_{d} l_{d}} \Delta \epsilon_{d} n_{d} .
$$

where $q$ is index of an atom in the primitive unit cell.

The essence of the next step is to use in DMFT or DMFT $+\Sigma$ loop (see section 2.1) the local lattice Green's function (5), determined by momentum integrated Dyson's equation of the form:

$$
\begin{gathered}
G_{q l m, q^{\prime} l^{\prime} m^{\prime}}(\omega)=\frac{1}{V_{B}} \int d \mathbf{k}\left[\omega \delta_{q l m, q^{\prime} l^{\prime} m^{\prime}}-\left(H_{\mathrm{LDA}}^{0}(\mathbf{k})\right)_{q l m, q^{\prime} l^{\prime} m^{\prime}}\right. \\
\left.+\delta_{q l, q_{d} l_{d}} \Sigma_{q l m, q^{\prime} l^{\prime} m^{\prime}}(\omega)\right]^{-1}
\end{gathered}
$$

where $[. . .]^{-1}$ denotes the inverse matrix with indices $n(=q l m), n^{\prime}\left(=q^{\prime} l^{\prime} m^{\prime}\right)$, while the integration is performed over the Brillouin zone of the volume $V_{B}$.

Significant simplification of computations is achieved for the case of cubic lattice symmetry, when the crystal field strongly splits $d$-orbitals into the three-fold degenerate $t_{2 g^{-}}$ states and two-fold degenerate $e_{g}$-states. In this special case both Green's function and self-energy become diagonal over orbital and spin indices. Then the calculation of local Green's function of the lattice problem can be performed as energy integration with the use of unperturbed density of states, which allows to avoid tedious integration over the Brillouin zone in (64) and write:

$$
G(\omega)=G^{0}(\omega-\Sigma(\omega))=\int d \epsilon \frac{N^{0}(\epsilon)}{\omega-\Sigma(\omega)-\epsilon} .
$$

In this case double counting correction $\Delta \epsilon_{d}$ reduces to immaterial shift of the chemical potential and its particular mathematical form is irrelevant.

\subsection{Examples of LDA+DMFT calculations.}

\subsubsection{Cubic perovskites $\mathrm{CaVO}_{3}$ and $\mathrm{SrVO}_{3}$}

In this section we consider examples of some LDA+DMFT calculations of electronic band structure of realistic compounds with strong enough electronic correlations. Transition metal oxides are ideal testing area to study electronic correlations in solids. Among these materials cubic perovskites have simplest crystal structure and thus can be viewed as a starting point to understand electronic properties of more complex systems. Usually $3 d$-states in such materials form comparatively narrow bands of the width $W \sim 2-3 \mathrm{eV}$, leading to strong electron-electron correlations. 
Modern stage of experimental investigations of spectral and transport properties of strongly correlated $3 d^{1}$ transition metal oxides started from the work of Fujimori et al. [123. The authors, apparently for the first time, discovered strongly pronounced lower Hubbard band in photoemission spectra, which could not be explained by standard methods of band structure calculations. In many of earlier works [124, 125, 126, 127], devoted to the properties of the series of compounds $\mathrm{Sr}_{1-x} \mathrm{Ca}_{x} \mathrm{VO}_{3}$ with different values of $x$ rather controversial results were reported. While thermodynamic characteristics (Sommerfeld coefficient, electric resistivity and magnetic susceptibility) appeared to be more or less $x$ independent, spectroscopic measurements data rather strongly changed as system transformed from $x=0\left(\mathrm{SrVO}_{3}\right)$ to $x=1\left(\mathrm{CaVO}_{3}\right)$. These data indicated a transition from strongly correlated metal $\left(\mathrm{SrVO}_{3}\right)$ to practically ideal insulator $\left(\mathrm{CaVO}_{3}\right)$, with concentration range $x \rightarrow 1$ in $\mathrm{Sr}_{1-x} \mathrm{Ca}_{x} \mathrm{VO}_{3}$ being the boundary of Mott-Hubbard transition. Analysis of this problem was performed using the high penetration depth photoemission experiments by Maiti et al. [128], and similar experiments with high resolution photoemission by Sekiyama et al. [129]. In particular, in the last work it was shown that (1) surface preparation technique is very important (preferable is cleavage method) and (2) energy of X-ray beam should be big enough to provide penetration depth of several elementary cells. At the same time high instrumental resolution should be guaranteed (about $100 \mathrm{meV}$ in the work [129]). Such improvement of photoemission methods lead to observation of almost identical spectra for $\mathrm{Sr}(\mathrm{Ca}) \mathrm{VO}_{3}$ [128, 129], demonstrating agreement of spectroscopic and thermodynamic measurements. Results of these experiments agree also with earlier 1s X-ray absorption spectra (XAS) obtained by Inoue et al. [130], which differ only for energies slightly below the Fermi level in contrast to BIS data [127]. In the framework of single band Hubbard model with neglect of orbital structure of $3 d$-shell of $\mathrm{V}$, Rozenberg et al. [131] modelled $\mathrm{Sr}_{1-x} \mathrm{Ca}_{x} \mathrm{VO}_{3}$ spectra obtained by high penetration depth photoemission [128] using adjustable parameters. Later in Ref. [129] it was demonstrated that data Ref. [128] contained quite significant surface contribution.

Below we present results of $\mathrm{LDA}+\mathrm{DMFT}(\mathrm{QMC})$ calculations, performed without any adjustable parameters, both for spectral function and density of states of cubic $\mathrm{SrVO}_{3}$ and orthorhombic $\mathrm{CaVO}_{3}$ perovskites. According to these both systems are strongly correlated metals, which are quite far away from metal-insulator transition boundary. Despite significantly smaller $\mathrm{V}-\mathrm{O}-\mathrm{V}$ bond angle in $\mathrm{CaVO}_{3}$, photoemission spectra of both systems are very similar and their quasiparticle peaks are almost identical. The results obtained agreed very well with modern high resolution bulk sensitive photoemission data, mentioned above. In the spectral function of $\mathrm{SrVO}_{3}$, obtained from LDA+DMFT(QMC) calculations, kinks of purely electronic nature at about $200 \mathrm{meV}$ were observed, and later these kinks were observed experimentally.

Results of LDA+DMFT calculations First of all from LDA calculated band structure we extract single electron Hamiltonian $\hat{H}_{\mathrm{LDA}}^{0}$ with subtracted average Coulomb interaction (to avoid double counting) [16]. Supplementing $\hat{H}_{\mathrm{LDA}}^{0}$ with local Coulomb interaction between electrons we obtain the Hamiltonian (59) for the material of interest. Since symmetry of $\mathrm{CaVO}_{3}$ is close to cubic one, it is possible to simplify the calculations and use integration with LDA density of states $N^{0}(\epsilon)$, instead of integration over the Brillouin zone. In the Hamiltonian (59) local intraorbital and interorbital repulsions and exchange interactions are taken into account explicitly as $U, U^{\prime}$ and $J$. The values of these interactions for $\mathrm{SrVO}_{3}$ were calculated by constrained LDA method [117] with $e_{g}$-states included 

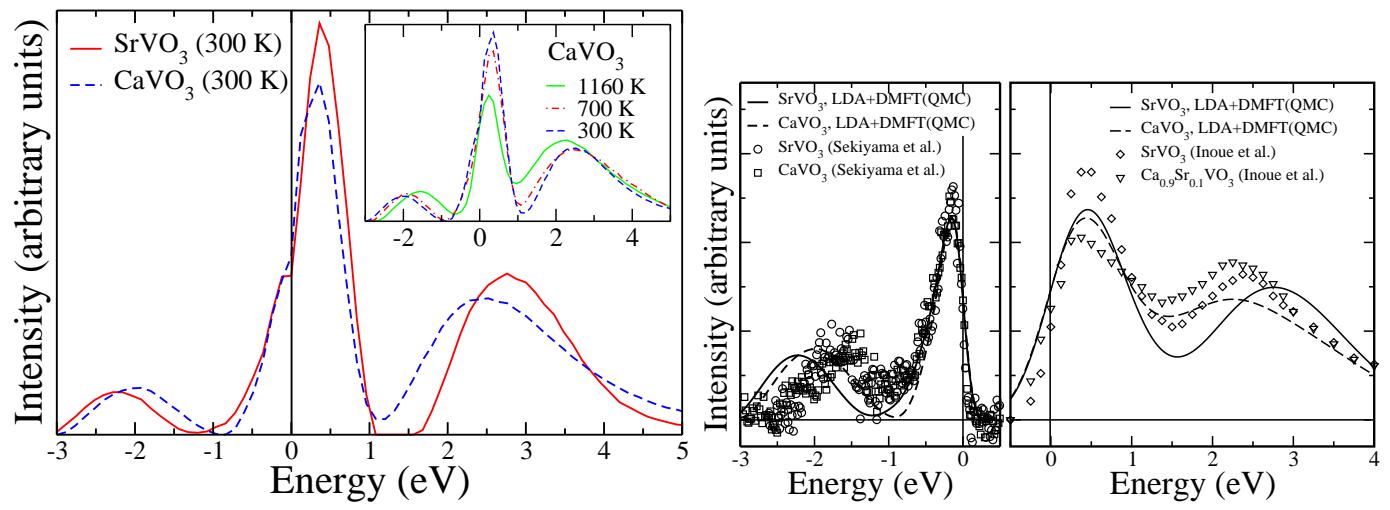

Figure 18: On the left side: $\mathrm{LDA}+\mathrm{DMFT}(\mathrm{QMC})$ spectra for $\mathrm{SrVO}_{3}$ (solid line) and $\mathrm{CaVO}_{3}$ (dashed line) at $\mathrm{T}=300 \mathrm{~K}$ (inset: temperature influence on $\mathrm{CaVO}_{3}$ spectrum lineshape). On the right side: comparison of calculated (without adjustable parameters) LDA+DMFT(QMC) spectra for $\mathrm{SrVO}_{3}$ (solid line) and $\mathrm{CaVO}_{3}$ (dashed line) with high resolution bulk sensitive photoemission data $\left(\mathrm{SrVO}_{3}\right.$ - circles; $\mathrm{CaVO}_{3}$ - rectangles) [129] (left picture) and $1 s$ - XAS-spectra: $\left(\mathrm{SrVO}_{3}\right.$ - diamonds, $\mathrm{Ca}_{0.9} \mathrm{Sr}_{0.1} \mathrm{VO}_{3}$ - triangles) [130] (right figure). Horizontal line - experimental background.

into screening [132]. Obtained value of averaged Coulomb interaction is $\bar{U}=3.55 \mathrm{eV}$ $\left(\bar{U}=U^{\prime}\right.$ for $t_{2 g}$ orbitals [17, 116]) and $J=1.0 \mathrm{eV}$. Intraorbital Coulomb repulsion $U$ is fixed by rotational invariance $U=U^{\prime}+2 J=5.55 \mathrm{eV}$. For $\mathrm{CaVO}_{3} \bar{U}$ was not calculated, since standard procedure of calculation of Coulomb interaction parameters between two $t_{2 g}$ electrons screened by $e_{g}$ states is not applicable for distorted crystal structure, where $e_{g}$ and $t_{2 g}$ orbital are not separated by symmetry. On the other hand it is known that changes of local Coulomb interaction are usually much smaller than changes in density of states, which as shown above are weakly dependent on bond angle V-O-V. It means that $\bar{U}$ for $\mathrm{CaVO}_{3}$ should be practically the same as for $\mathrm{SrVO}_{3}$. Correspondingly, the values $\bar{U}=3.55 \mathrm{eV}$ and $J=1.0 \mathrm{eV}$ were used for both $\mathrm{SrVO}_{3}$ and $\mathrm{CaVO}_{3}$. These values agree with other band structure calculations for vanadium compounds [132] and experimental data [133].

Further calculations with Hamiltonian (59) were performed in the framework of DMFT with quantum Monte-Carlo method (QMC) [13] as an impurity solver. In QMC the Green's function was obtained for the imaginary time and then continued on the real time (frequency) by maximum entropy method [134]. In LDA+DMFT(QMC) calculated spectra for $\mathrm{SrVO}_{3}$ and $\mathrm{CaVO}_{3}$ shown in Fig. 18 (on the left side) we observe manifestations of correlation effects, such as formation of lower Hubbard bands near $-1.5 \mathrm{eV}$ and upper Hubbard bands at about $2.5 \mathrm{eV}$ with well developed quasiparticle peaks on the Fermi level. Thus both $\mathrm{SrVO}_{3}$ and $\mathrm{CaVO}_{3}$ are strongly correlated metals. Difference of bare bandwidth (about 4\%) only leads to a small additional spectral weight transfer from quasiparticle peak to Hubbard bands and slight changes of Hubbard bands positions. Obviously, both systems are close to Mott-Hubbard metal insulator transition. Manybody densities of states for both systems (Fig. 18) are similar but not identical. Indeed, $\mathrm{SrVO}_{3}$ is a bit less correlated than $\mathrm{CaVO}_{3}$ in agreement with difference of the LDA bandwidths. The inset in Fig. 18 shows that temperature influence on the spectrum is small for $T \lesssim 700 \mathrm{~K}$.

In the middle part of Fig. $18 \mathrm{LDA}+\mathrm{DMFT}(\mathrm{QMC})$ densities of states (obtained at $T=300 \mathrm{~K}$, multiplied with the Fermi function at $20 \mathrm{~K}$ and broadened with Gaussian of the 
width $0.1 \mathrm{eV}$ to mimic experimental resolution [129]) are compared with experimental photoemission data after subtraction of oxygen and surface contributions. In particular, the height and the width of these spectra are almost the same in $\mathrm{SrVO}_{3}$ and $\mathrm{CaVO}_{3}$ (with a bit of difference above the Fermi level). On the other hand, positions of lower Hubbard band differs quite noticeably. This distinctions might occur because of subtraction of (estimated) oxygen contribution, which can delete part of $3 d$ spectral weight below $-2 \mathrm{eV}$, as well as with uncertainties of $\bar{U}$ calculations.

On the right side of Fig. 18 we show comparison with XAS data. Finite lifetime effects for holes are taken into account by broadening of theoretical spectra with Lorentzian of the width of $0.2 \mathrm{eV}$ [135, multiplication by inverse Fermi function (at $T=80 \mathrm{~K}$ ) and further broadening with Gaussian for experimental resolution $0.36 \mathrm{eV}$. General agreement of weights and positions of quasiparticle and upper Hubbard bands for $t_{2 g}$-band is good, including tendencies associated with transition from . $\mathrm{SrVO}_{3}$ to $\mathrm{CaVO}_{3}$ (in the experiment $\left.\mathrm{Ca}_{0.9} \mathrm{Sr}_{0.1} \mathrm{VO}_{3}\right)$. For $\mathrm{CaVO}_{3}$ quasiparticle spectral peak weight is a bit smaller than in the experiment. In contrast to single band Hubbard model calculations LDA+DMFT accounts for peculiarities of the systems and reproduce strong asymmetry of the spectra close to the Fermi energy, including relative weights and bandwidths. These results give different interpretation of XAS as compared with Ref. [130], where maximum at 2.5 $\mathrm{eV}$ was associated with $e_{g}$ band and not with upper Hubbard band of $t_{2 g}$ band. Small differences of qusiparticle peaks (see Fig. 18) lead to different values of effective masses: $m^{*} / m_{0}=2.1$ for $\mathrm{SrVO}_{3}$ and $m^{*} / m_{0}=2.4$ for $\mathrm{CaVO}_{3}$. These theoretical values agree with $m^{*} / m_{0}=2-3$ for $\mathrm{SrVO}_{3}$ and $\mathrm{CaVO}_{3}$ obtained from de Haas-van Alphen experiments and thermodynamic data [124, 125, 126, 136]. Note that the effective mass for $\mathrm{CaVO}_{3}$ determined from optical experiments is slightly larger: $m^{*} / m_{0}=3.9$ [133].

\subsubsection{Kinks in spectral function of $\mathrm{SrVO}_{3}$.}

Let us consider in detail $\mathrm{LDA}+\mathrm{DMFT}(\mathrm{QMC})$ results for spectral function $A(\mathbf{k}, \omega)$ for $\mathrm{SrVO}_{3}$ obtained in Ref. [92]. Owing to ideal cubic lattice symmetry self-energy matrix $\Sigma(\omega)$ is diagonal and all diagonal elements are the same for all $t_{2 g}$ orbitals. Spectral function is defined by imaginary part of Green function $\operatorname{Im} G(\mathbf{k}, \omega)$, i.e. in fact by selfenergy $\Sigma(\omega)$ on real axis. This self-energy was calculated by numerical solution of Dyson's equation for the known interacting and bare Green functions as described in the Appendix of Ref. [122].

In the Fig. 19 this self-energy $\Sigma(\omega)$ is plotted as a function of real frequencies. It is essentially asymmetric with respect to the Fermi level, as could be assumed from the asymmetry of LDA density of states and band filling $1 / 6$. At energies $\omega \sim \pm 1.5 \mathrm{eV}$ the real part of self-energy has extrema, corresponding to transition region from quasiparticle peak to lower and upper Hubbard bands. Two extrema in the imaginary part of the self-energy coinciding with $\operatorname{Re} \Sigma$ zeros 7 determine energy positions of lower and upper Hubbard bands (see Fig. 18).

Asymmetric quasiparticle peak in the density of states (DOS) is situated in the energy range $-0.8-1.4 \mathrm{eV}$ Fig. 18, We see that imaginary part of self-energy $\operatorname{Im} \Sigma(\omega)$ is sufficiently small for these energies, while the real part can be roughly approximated with the dashed straight line shown in Fig. 19). The slope of this line defines mass renormalization value

\footnotetext{
${ }^{7}$ Here we remind that real part of self-energy is connected with its imaginary part via Kramers-Kronig relation 91 .
} 


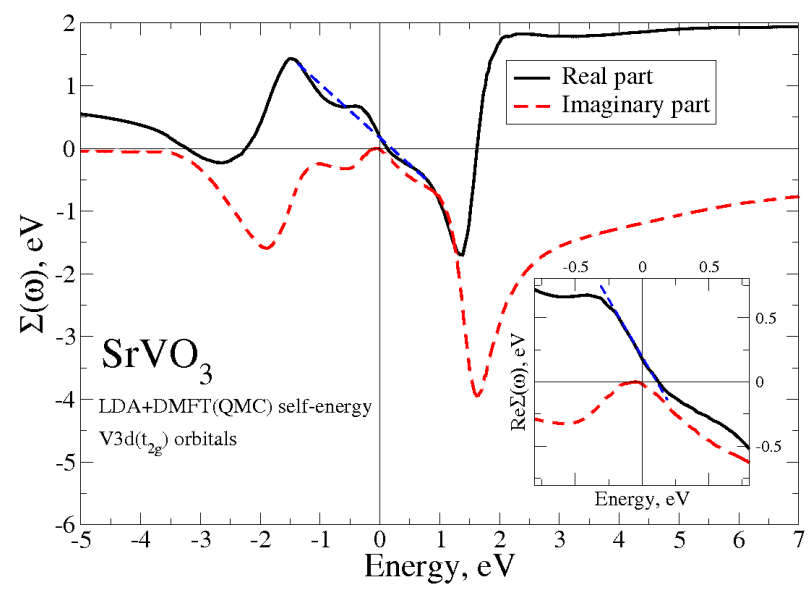

Figure 19: Real (black solid line) and imaginary (grey line) parts of self-energy $\Sigma(\omega)$ obtained from LDA+DMFT(QMC) calculation for V3d- $t_{2 g}$ orbitals of $\mathrm{SrVO}_{3}[92$. In the inset self-energy in the vicinity of the Fermi level is shown in more details. Dashed lines give the slope of $\operatorname{Re} \Sigma(\omega)$ far away and close to the Fermi level.

$Z=m^{\star} / m=1-\left.\frac{\partial \operatorname{Re} \Sigma(\omega)}{\partial \omega}\right|_{\omega=0}=1.9$. Such $Z$ value agrees with one obtained from QMC data in Matsubara frequencies: $m^{\star} / m=1-\frac{\operatorname{Im} \Sigma\left(\omega_{0}\right)}{\omega_{0}} \approx 2$, where $\omega_{0}$ is "zeroth" Matsubara frequency. This value of renormalization is in accord with the value $m^{*} / m=2.2$ from the works [137, 138] as well as with experimental estimate from ARPES data [139].

In the inset in Fig. 19 it is visible that Fermi liquid behavior of self-energy $\operatorname{Im} \Sigma(\omega) \sim$ $-\omega^{2}$, together with $\operatorname{Re} \Sigma(\omega) \sim-\omega$, is fulfilled only in the interval from -0.2 to $0.15 \mathrm{eV}$. The slope of $\operatorname{Re} \Sigma(\omega)$ in immediate proximity to the Fermi level is steeper than in wider energy interval (dashed line in Fig. 19). Thus Fermi liquid mass renormalization value is larger than $m^{*} / m=1.9$ and is equal to $m_{\mathrm{low} E}^{*} / m=3$ (dashed line on the inset of Fig. 19). On the edges of Fermi liquid regime, sharp bends of $\operatorname{Re} \Sigma(\omega)$ at energies $\omega= \pm 0.25 \mathrm{eV}$ are seen. As the border of Fermi liquid regime we can consider energies, where the self-energy behavior starts to differ from $\operatorname{Im} \Sigma(\omega) \sim-\omega^{2}$, which because of Kramers-Kronig relation corresponds to $\operatorname{Re} \Sigma(\omega) \sim-\omega$. Deviation from the square behavior of $\operatorname{Im} \Sigma$ at energies of the order of $\omega= \pm 0.25 \mathrm{eV}$ immediately leads to cusps in the $\operatorname{Re} \Sigma(\omega)$.

If the self-energy on the real axis is known one can compute spectral function $A(\mathbf{k}, \omega)$ and also the quasiparticle dispersion determined by momentum dependence of its maxima. In Fig. 20 we show the map of the spectral density for $\mathrm{SrVO}_{3}$ obtained in Ref. [92]. In this multiband system (with degenerate bands) further analysis is similar to that for the single band case of section 3.3.2. White dots denote dispersion curves $E_{n \mathbf{k}}$ obtained from LDA+DMFT calculation for $\mathrm{SrVO}_{3}$. In the narrow vicinity of the Fermi level they coincide with LDA band structure $\epsilon_{n \mathbf{k}}$ (line 1) renormalized by Fermi liquid factor $Z_{\mathrm{FL}}=$ 0.35 , so that $E_{n \mathbf{k}}=Z_{\mathrm{FL}} \epsilon_{n \mathbf{k}}$ (line 2). Outside the Fermi liquid region dispersion curves correspond to LDA band structure with different renormalization factor: $E_{n \mathbf{k}}=Z_{\mathrm{CP}} \epsilon_{n \mathbf{k}}+$ $c_{ \pm}$(line 3), where $Z_{\mathrm{CP}}=0.64, c_{+}=0.086 \mathrm{eV}, c_{-}=0.13 \mathrm{eV}$. Along high-symmetry $\Gamma-\mathrm{M}$ and $\Gamma-\mathrm{R}$ directions in the Brillouin zone, transition between these two regimes leads to formation of kinks in the effective dispersions at energies $\omega_{\star,+}=0.22 \mathrm{eV}$ and $\omega_{\star,-}=-0.24$ $\mathrm{eV}$. These kinks are marked with arrows on the right side of Fig. 20, which corresponds to the area surrounded with the white rectangle on the main part of the figure. On the 


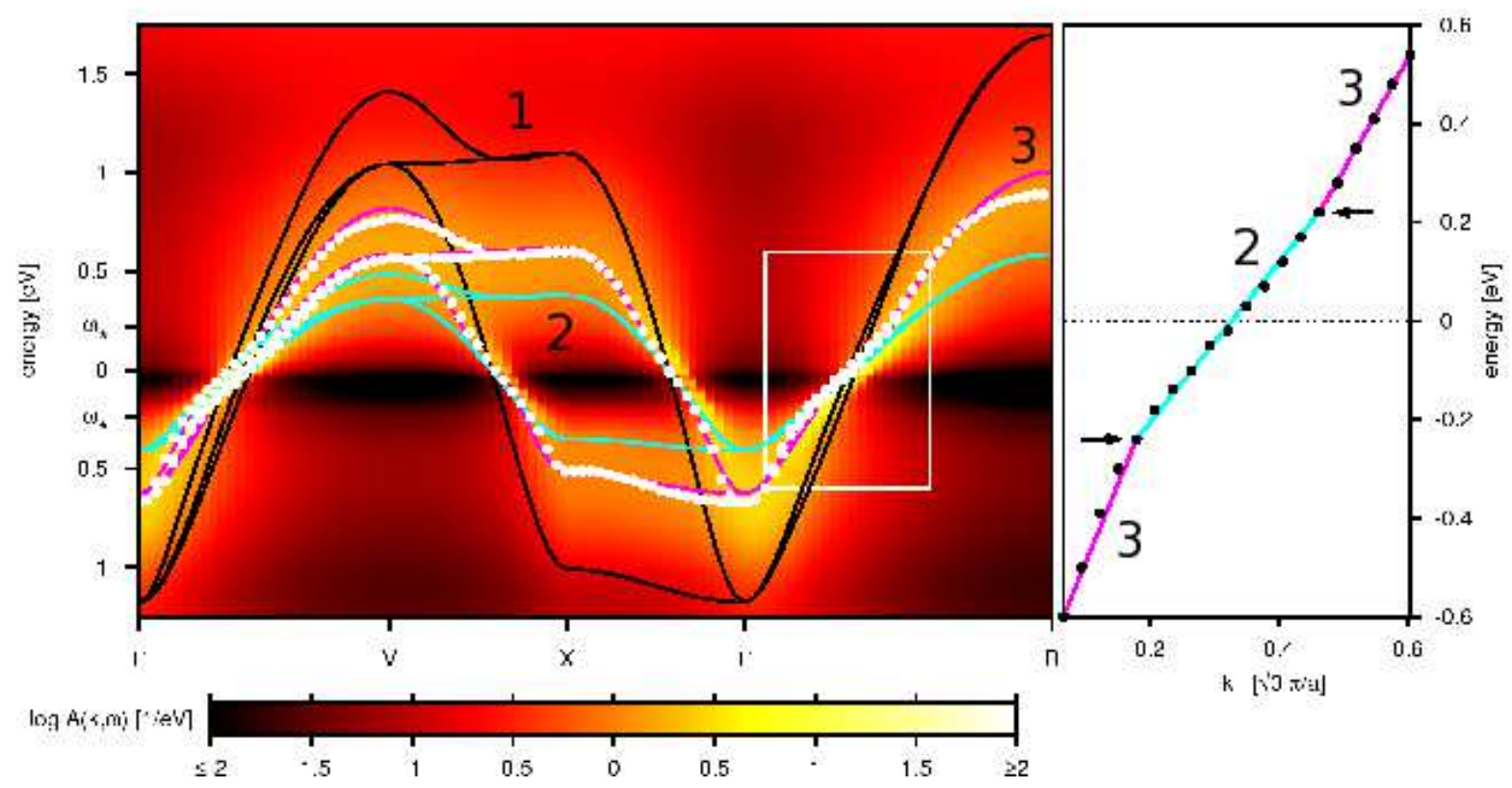

Figure 20: Kinks in the dispersion relation $E_{n \mathbf{k}}$ (white dots), for $\mathrm{SrVO}_{3}$ obtained from LDA+DMFT calculation. Line 1 - LDA band structure $\epsilon_{n \mathbf{k}}$; line 2 - LDA bands renormalized with Fermi liquid mass renormalization factor. lines 3 - band structure within the intermediate regime. Kinks are marked with arrows on the right panel, which corresponds to the area restricted with white rectangle on the main part of the figure.

contour plot of spectral function $A(\mathbf{k}, \omega)$ it is seen that in the energy region sufficiently far away from the Fermi level, spectral function keeps explicit k-dependence, despite pretty large damping value, replacing traditional band structure picture for systems with strong electron-electron correlations.

Kinks of electronic nature were discovered in this system in ARPES experiments [139] in $\Gamma-\mathrm{M}$ direction at energies of the order of $0.25 \mathrm{eV}$, which agrees quite well with results of LDA+DMFT(QMC) calculations.

\subsection{Electronic structure of copper oxides in the pseudogap state: $\mathrm{LDA}+\mathrm{DMFT}+\Sigma$.}

Pseudogap state as was already pointed above is one of main anomalies of the normal state of high- $T_{c}$ cuprates and it is thought that clarification of its physical nature is the key point to understand high-temperature superconductivity mechanism [38, 39, 140]. Most powerful tool to investigate this state in recent years became angular resolved photoemission (ARPES). During last ten years in this area there was a remarkable progress related to significant growth of ARPES resolution both in energy and in momentum space [141, 142]. From ARPES data the Fermi surface (FS) shape, quasiparticle dispersion and damping, even self-energy can be directly restored [141, 142]. This allowed to study in detail formation of the pseudogap, "shadow" bands, quite unusual phenomena of Fermi arcs formation, interlayer hybridization effects (bilayer splitting) in double layer systems, [141, 142], to determine qualitative distinctions between electron and hole doped cuprates [141, 142]. The purpose of theory is an explanation of all these peculiarities and this problem is much complicated by rather strong electronic correlations, typical for these 
systems and making doubtful the standard band theory and Fermi liquid approach.

In this section we shall demonstrate that an account of AFM short range order fluctuations is in principle enough to describe a number of ARPES experiments in real systems. To this purpose we use LDA + DMFT $+\Sigma$ hybrid computational scheme [143, 144, 145, 146, 147, 148. On the one hand this scheme inherits all advantages of LDA+DMFT [16, 17, 18, 19, 20], i.e. the combination of single electron first principle density functional theory within the local density approximation (DFT/LDA) [108, 149] with dynamical mean-field theory (DMFT) for strongly correlated electrons [9, 3, 4, 5, 7]. On another hand this scheme allows to consider nonlocal correlations by introduction of momentum dependent self-energy, while the usual self-consistent set of DMFT equations is preserved [23, 24, 25]. To solve effective single impurity problem of DMFT in the works described below we employed numerical renormalization group (NRG) [14, 15].

Such computational scheme fits very well to describe electronic properties of high- $\mathrm{T}_{c}$ cuprates in normal (underdoped) state. Firstly, all material specific model parameters of physically relevant $\mathrm{Cu}-3 \mathrm{~d} x^{2}-y^{2}$ orbital can be obtained from LDA calculations. Secondly, stoichiometric cuprates are antiferromagnetic Mott insulators with $U \gg W$ ( $U$ - local Coulomb interaction $W$ - conduction band width), so that correlation effects there are very important. At finite doping (at least up to optimal doping) cuprates are typical strongly correlated metals and DMFT stage of the computational scheme allows one to account for strong electronic correlations. Finally, to study "antiferromagnetic scenario" of pseudogap formation we introduce into the standard LDA+DMFT scheme k- dependent self-energy $\Sigma_{\mathbf{k}}$, describing nonlocal correlations induced by (quasi) static Heisenberg spin fluctuations of short range AFM order [41, 42].

In the framework of $\mathrm{LDA}+\mathrm{DMFT}+\Sigma$ approach we performed calculations for a series of high temperature superconductors: hole doped $\mathrm{Bi}_{2} \mathrm{Sr}_{2} \mathrm{CaCu}_{2} \mathrm{O}_{8-\delta}(\mathrm{Bi} 2212)$ [143] and $\mathrm{La}_{2-x} \mathrm{Sr}_{x} \mathrm{CuO}_{4}$ (LSCO) [144], and also for electron doped $\mathrm{Nd}_{2-x} \mathrm{Ce}_{x} \mathrm{CuO}_{4}$ (NCCO) [145, 146] and $\mathrm{Pr}_{2-x} \mathrm{Ce}_{x} \mathrm{CuO}_{4}$ (PCCO) [147]. LDA $+\mathrm{DMFT}+\Sigma$ calculation results for Fermi surfaces and spectral functions can be compared with ARPES data for quasiparticles bands and experimental Fermi surface maps.

Crystal structure of Bi2212 [143], NCCO [145, 146] and PCCO [147] have tetragonal symmetry with the space group I4/mmm, while LSCO has distorted orthorhombic structure Bmab [144]. In more details crystallographic data used in LDA + DMFT $+\Sigma$ calculations are presented in Refs. [143, 144, 145, 146, 147, 148].

It is well known that physical properties of cuprates in many respects are determined by quasi-two-dimensionality of their electronic properties. From this point of view, the main interest is attracted to electronic states of $\mathrm{CuO}_{2}$ plane, where we are dealing with partially filled antibonding $\mathrm{Cu}-3 d\left(x^{2}-y^{2}\right)$ orbitals with dispersion crossing the Fermi level. In tight-binding approximation this dispersion has the following form:

$$
\begin{aligned}
\varepsilon(\mathbf{k})= & -2 t \quad\left(\cos k_{x} a+\cos k_{y} a\right)-4 t^{\prime} \cos k_{x} a \cos k_{y} a \\
& -2 t^{\prime \prime} \quad\left(\cos 2 k_{x} a+\cos 2 k_{y} a\right)-2 t^{\prime \prime \prime}\left(\cos k_{x} a \cos 2 k_{y} a+\cos 2 k_{y} a \cos k_{y} a\right) .
\end{aligned}
$$

Here $t, t^{\prime}, t^{\prime \prime}, t^{\prime \prime \prime}$ are $\mathrm{Cu}-\mathrm{Cu}$ transfer integrals in first four coordination spheres in the $\mathrm{CuO}_{2}$ plane, $a$ is the lattice constant. Values of these effective transfer integrals calculated with the use of Wannier functions obtained within the N-th order muffin-tin orbitals method (NMTO) of Ref. [121] are listed in the Table 1. In the following we shall exploit LDA calculated effective antibonding $\mathrm{Cu}-3 d\left(x^{2}-y^{2}\right)$ band as a "bare" one in $\mathrm{LDA}+\mathrm{DMFT}+\Sigma$ calculations. 
Table 1: Calculated model energy parameters $(\mathrm{eV})$ and experimental correlation length $\xi$. First four $\mathrm{Cu}-\mathrm{Cu}$ transfer integrals in the $\mathrm{CuO}_{2}$ plane $t, t^{\prime}, t^{\prime \prime}, t^{\prime \prime \prime}$; effective interlayer transfer integral $t_{\perp}$, local Coulomb interaction $U$ and pseudogap amplitude $\Delta$.

\begin{tabular}{|c|c|c|c|c|c|c|c|c|}
\hline & $t$ & $t^{\prime}$ & $t^{\prime \prime}$ & $t^{\prime \prime \prime}$ & $t_{\perp}$ & $U$ & $\Delta$ & $\xi$ \\
\hline Bi2212 & -0.627 & 0.133 & 0.061 & -0.015 & 0.083 & 1.51 & 0.21 & $10 \mathrm{a}$ \\
\hline NCCO & -0.44 & 0.153 & 0.063 & -0.01 & - & 1.1 & 0.36 & $50 \mathrm{a}$ \\
\hline PCCO & -0.438 & 0.156 & 0.098 & - & - & 1.1 & 0.275 & $50 \mathrm{a}$ \\
\hline LSCO & -0.476 & 0.077 & -0.025 & -0.015 & - & 1.1 & 0.21 & $10 \mathrm{a}$ \\
\hline
\end{tabular}

In double layer systems, e.g. in Bi2212, hopping between two neighboring planes is also important. In tight-binding approximation an expression for corresponding interlayer dispersion derived in Ref. [150] has the form:

$$
t_{\perp}(\mathbf{k})=\frac{t_{\perp}}{4}\left(\cos k_{x} a-\cos k_{y} a\right)^{2}
$$

The value of $t_{\perp}$ is given in Table 1, Consideration of interlayer hopping and "bilayer splitting" effects requires certain generalization of LDA $+\mathrm{DMFT}+\Sigma$ computational scheme [143].

To perform DMFT calculations one should also calculate a value of onsite Coulomb interaction. The value of this interaction for effective $\mathrm{Cu}-3 d\left(x^{2}-y^{2}\right)$ orbital obtained within the constrained LDA method [117] is also given in Table 1.

To account for AFM spin fluctuations we employed two-dimensional model of the pseudogap state [41, 42], generalized for DMFT $+\Sigma$ calculations [23, 25]. Additional "external" k-dependent self-energy $\Sigma_{\mathbf{k}}[23,25]$ describes nonlocal correlations caused by (quasi)static 8 AFM spin fluctuations.

To specify $\Sigma_{\mathbf{k}}$ it is necessary to know two important parameters - the pseudogap amplitude $\Delta$, giving energy scale of fluctuating SDW and correlation length $\xi$. The value of $\Delta$ were calculated as described in Refs. [23, 25, 143]. The values of correlation length were taken in accordance with values obtained in neutron scattering experiments for NCCO [151] and LSCO [152. The values of $\Delta$ and $\xi$ used for all systems under consideration are also listed in Table 1. To solve effective Anderson single impurity problem in DMFT we used numerical renormalization group (NRG [14, 15]). Temperature in DMFT(NRG) calculations was chosen to be $0.011 \mathrm{eV}$ and electron or hole concentration (doping level) was taken to be $15 \%$.

$\mathrm{LDA}+\mathrm{DMFT}+\Sigma$ calculations produce a clear picture of "hot spots" behavior in the spectral function and on maps of the Fermi surfaces for electron doped systems [145, 146, 147], while for hole doped systems only Fermi arcs arise [143, 144].

In Fig. 21 LDA + DMFT $+\Sigma$ we show spectral functions along 1/8 part of bare Fermi surface from nodal point on the diagonal of the Brillouin zone (upper curve) downto antinodal point at the boundary of the zone (lower curve). Results for Bi2212 are shown on the left panel and for NCCO on the right panel of Fig. 21. For both systems in nodal direction quasiparticles are well defined - sharp peak of spectral function situated

\footnotetext{
${ }^{8}$ Quasistatic approximation for AFM fluctuations necessarily restricts this approach to rather high temperatures (and energies not very close to the Fermi level) 41, 42. Thus we can not judge about the nature of low temperature (low energy) damping which is determined by dynamical (inelastic) scattering processes.
} 

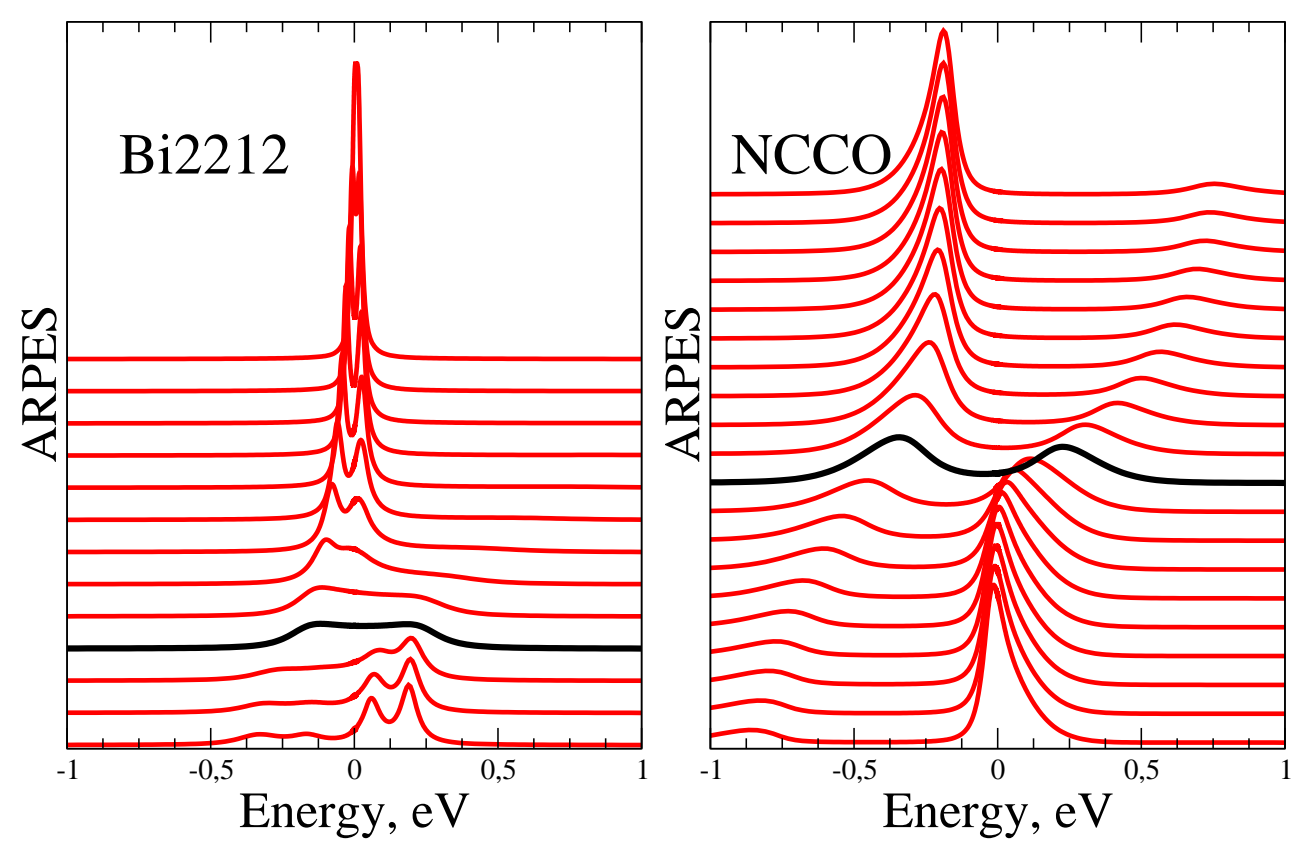

Figure 21: LDA + DMFT $+\Sigma$ spectral functions for Bi2212 (leftpanel) and NCCO (right panel) along the "bare" Fermi surface in the 1/8th of the Brillouin zone. Black line corresponds to the "hot spot" 145, 146]

practically on the Fermi level is clearly seen. As one moves to antinodal point quasiparticle damping grows reaching the maximum at the "hot-spot" and the peak of spectral density moves away from the Fermi level. This behavior is in complete agreement with results of Refs. [153, 154] (comparison with experiment see in [145, 146]). From LDA+DMFT+ $\Sigma$ results shown in Fig. 21 it is directly seen, that for Bi2212 antinodal states are formed by low energy edge of the pseudogap 9 , while for NCCO by high energy edge. For Bi2212 we also observe bilayer splitting of quasiparticle peak which is related to the presence of two $\mathrm{CuO}_{2}$ planes in the elementary cell.

The "hot spots" for NCCO are located closer to the Brillouin zone diagonal [145, 146. This can be seen from black lines on Fig. 21, which correspond to the "hot spots". Moreover, correlation length in NCCO is much larger than in Bi2212. Thus for NCCO (in contrast to Bi2212) in the antinodal direction quasiparticles again are rather well defined. For Bi2212 scattering near by Brillouin zone boundaries is strong everywhere and instead of "hot spots" picture we observe quite strong "destruction" of the Fermi surface close to these boundaries. Qualitatively the same picture is observed also in LSCO.

In Fig. 22, on the upper panel, LDA $+\mathrm{DMFT}+\Sigma$ Fermi surface maps in the quarter of the Brilloun zone for Bi2212 (on the left) and NCCO (on the right) are presented. In Bi2212 we observe strong "destruction" of Fermi surface by scattering by pseudogap fluctuations close to Brillouin zone boundaries 10 . On the contrary, in NCCO the Fermi surface is almost recovered close to the Brillouin zone boundaries. On the other hand Fermi arc around nodal direction in Bi2212 is clearly pronounced, while for NCCO it is noticeably smeared. This is another consequence of the fact that in NCCO "hot spots"

\footnotetext{
${ }^{9}$ Especially clear it is visible for the case of smaller correlation length $\xi=5 a$ considered in Ref. 143.

${ }^{10}$ Analogous behavior in entire accordance with ARPES results is realized also in another hole doped system - LSCO. The LDA + DMFT $+\Sigma$ calculations for this system were performed in [144]
} 

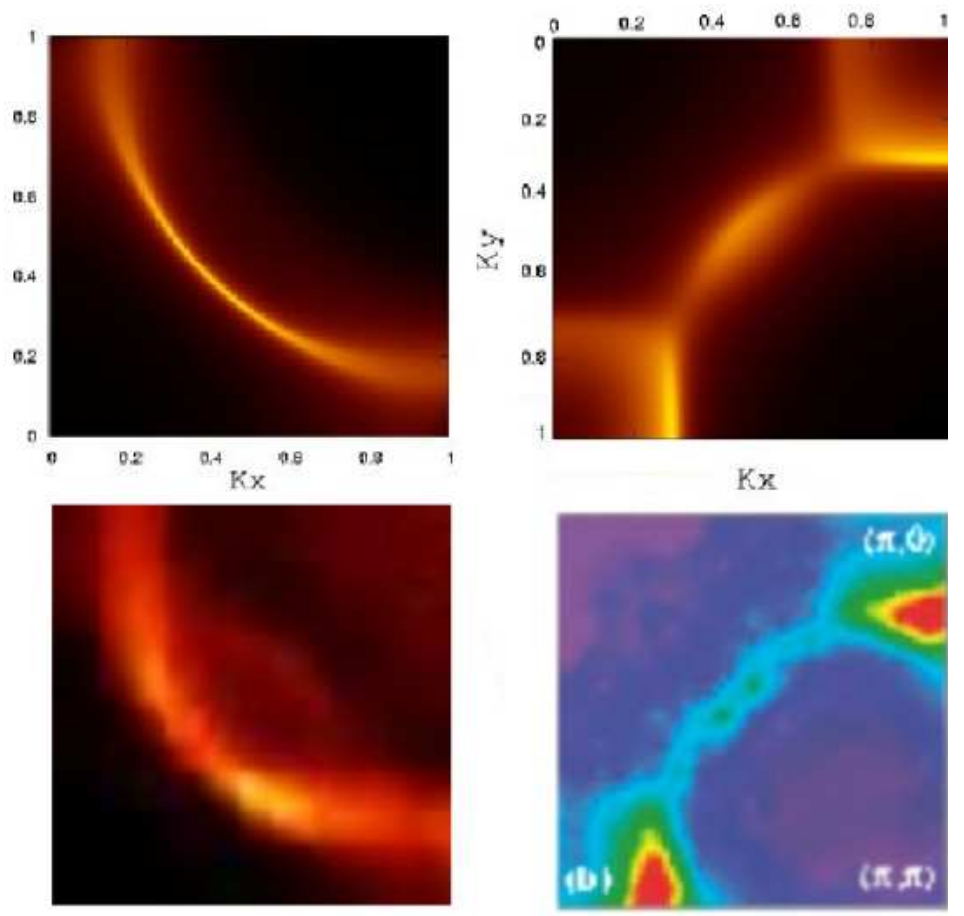

Figure 22: LDA+DMFT $+\Sigma$ Fermi surface maps obtained in Refs. [145, 146] for Bi2212 (upper left panel) and NCCO (upper right panel) in the quarter of the Brillouin zone, $\left(k_{x}, k_{y}\right.$ are given in units of $\pi / a)$. Experimental Fermi surface for Bi2212 (lower left panel [155]) and NCCO (lower right panel [153]).

are located closer to the Brillouin zone diagonal. A bit larger value of the pseudogap amplitude $\Delta$ also favours the stronger damping of Fermi arcs in NCCO. One should note the appearance of the "shadow" Fermi surface, which is much more intensive for NCCO.

Qualitatively the same Fermi surfaces were observed experimentally in real $\mathrm{Bi}$ [155] and $\mathrm{Nd}[153]$ systems (lower panel of Fig. 22). Thus, the distinction of Fermi surface maps for these systems is related mainly to the distinction of band structure parameters of these materials. In particular, LDA Fermi surfaces of NCCO are more bended and "hot spots" appear to be rather far from the Brillouin zone boundaries, consequently the Fermi surface in the vicinity of these boundaries almost does not feel scattering by AFM fluctuations. In Bi2212 LDA Fermi surface is rather close to the Brillouin zone boundaries and $(\pi / a, 0)$ point, so that "hot spots" are also close to this point. Thus in Bi2212 they are more "washed away" by strong pseudogap scattering close to $(\pi / a, 0)$ point and are not observed. "Hot spots" in NCCO are more vivid also because of much larger value of correlation length of fluctuations.

Not less graphic results were obtained in $\mathrm{LDA}+\mathrm{DMFT}+\Sigma$ calculations and ARPES experiments for [147]. In Fig. 23 we show PCCO Fermi surface map (panel (a) $\mathrm{LDA}+\mathrm{DMFT}+\Sigma$ results, panel (b) - experimental ARPES data). Fermi surface here is clearly distinguishable only near Brillouin zone boundaries and around $(\pi / a / 2, \pi / a / 2)$ point (Fermi arc). Again, as in NCCO we observe "destruction" of the Fermi surface in "hot spots", located at the intersection of Fermi surface and its AFM shadow "replica" is detected. This "destruction" of the Fermi surface is due to the strong electron scattering by AFM spin (pseudogap) fluctuations. The "shadow" Fermi surface is observed, as it 

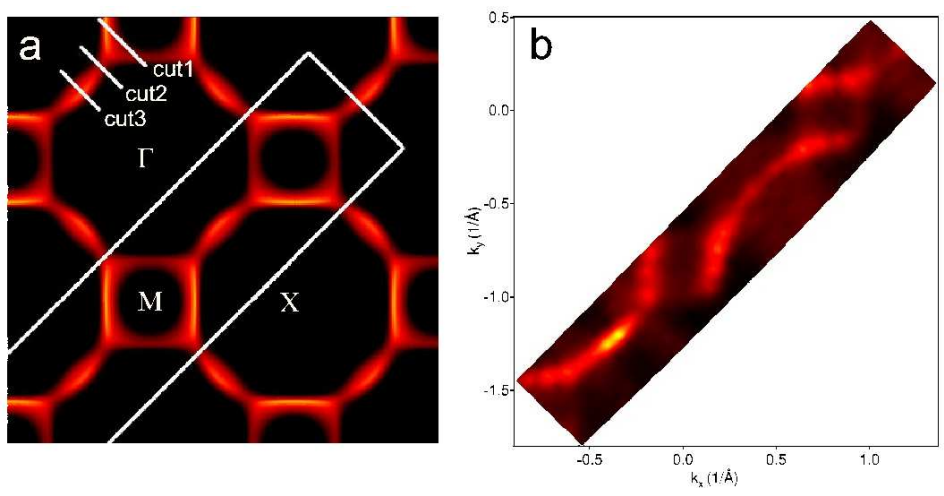

Figure 23: Fermi surface map for PCCO. (a) - results of LDA + DMFT $+\Sigma$ calculation. White rectangle on the panel (a) shows the part of reciprocal space where ARPES measurements (panel b) were done. Lower left corner coincides with X-point $(\pi / a, 0)$ of the Brillouin zone [147].

happens in the case of AFM doubling of the lattice period. However, since there is no long-range order in the underdoped region, in which we are interested, this "shadow" Fermi surface is strongly eroded. Fermi surface of PCCO is very similar to that observed in $\mathrm{Nd}_{2-x} \mathrm{Ce}_{x} \mathrm{CuO}_{4}$ (NCCO), which belongs to the same family of superconductors [145, 146, 153].

Let us compare (see Fig. 24) theoretical (upper panel) and experimental (lower panel) quasiparticle dispersions along most characteristic cuts of the Fermi surface shown in Fig. 23. Theoretical data are multiplied by the Fermi function with a temperature of 30K and convoluted (in energy) with a Gaussian distribution to simulate the experimental resolution. Cut 1 crosses the quasiparticle and the "shadow" Fermi surface near the Brillouin zone boundary. Correspondingly, here it is possible to detect "fork"-like structure, formed by suppressed "shadow" band and much better defined quasiparticle band. This structure corresponds to the beginning of formation of the Fermi surface cylinder around $(\mathrm{pi} / \mathrm{a}, 0)$ point. Cut 2 passes exactly through the "hot spot". Here we see a strong suppression of the quasiparticle band near the Fermi level. Cut 3 crosses the Fermi arc and we can see fairly well-defined quasiparticle band. However, the "shadow" band of low intensity is also present. In the case of long-range AFM order and a full doubling of the period, the Fermi surface and its "shadow" form a closed "pocket", of the Fermi surface around the $(\pi / 2 a, \pi / 2 a)$ point. while in the present case a part of the pocket formed by "shadow" band is strongly blurred. One can see that there is a good agreement between calculated and experimental data.

As was already noted, within $\mathrm{LDA}+\mathrm{DMFT}+\Sigma$ scheme two-particle properties can also be calculated [26, which allowed to investigate optical conductivity of $\mathrm{Bi}$ and $\mathrm{Nd}$ cuprates [145, 146], also demonstrating significant differences in the effects of pseudogap fluctuations. In particular, in optical conductivity of NCCO, in qualitative agreement with experiment [157], we observe a characteristic pseudogap dip and a smooth maximum due absorption through the pseudogap at frequencies $\sim 2 \Delta$. However, in optical conductivity of Bi2212 characteristic pseudogap structure practically does not occur neither in theory or in experiments [158], which is related to sufficiently small values of $\Delta$ and fluctuation correlation length in this system.

Let us summarize some of our conclusions. For all the systems studied, LDA $+\mathrm{DMFT}+\Sigma$ calculations show, that Fermi-liquid behavior persists only rather far away from the "hot 

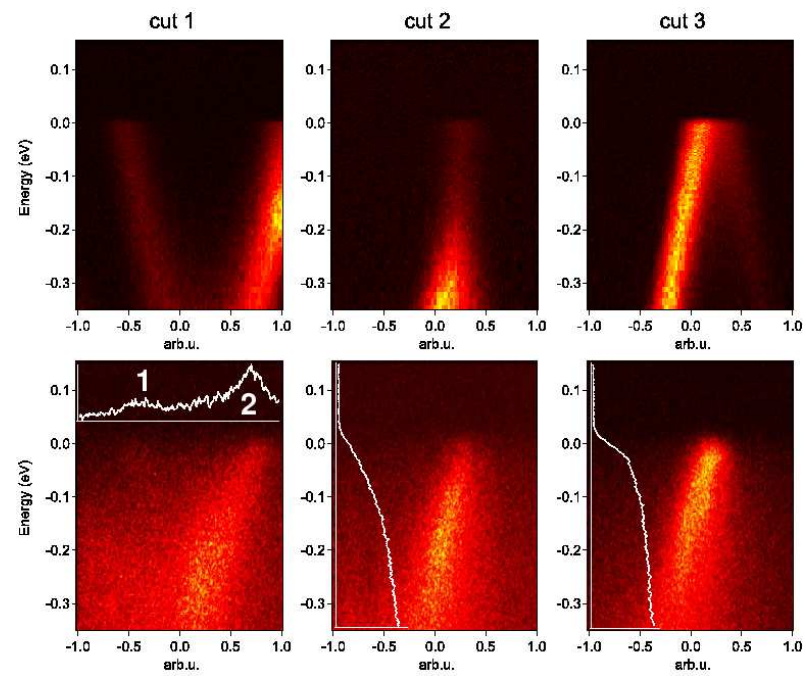

Figure 24: Momentum - energy distribution curves for characteristic cuts of the Brillouin zone plotted in Fig. 23 (upper panels - theoretical data, lower panels - experimental photoemission intensity). For the cut 1 we show momentum distribution curve (MDC) integrated over energy window of the width of $60 \mathrm{meV}$ around the Fermi level. Analogous MDC for the cut 2 (through the "hot spot") shows ARPES intensity suppression as compared with MDC for the cut 3, which is located quite far away from the "hot spot" [145, 146].

spots" (nodal direction), and "destruction" of the Fermi surface is observed near the "hot spots". This destruction is due to strong scattering of correlated electrons on short-range order AFM (pseudogap) fluctuations. Comparison of ARPES data and LDA + DMFT $+\Sigma$ calculations shows the existence of quite distinct "hot spots" in the behavior of the spectral density and maps of the Fermi surface in electron doped systems, in contrast to hole doped systems, where we only observe only a strong "destruction" of the Fermi surface near Brillouin zone boundaries and Fermi arc around its diagonal. There are several reasons for this difference: (1) "hot spots" in electron doped systems are located closer to the center of the Brillouin zone, (2) correlation length of AFM fluctuations in electron doped systems is bigger; (3) the width of the pseudogap in the electron doped systems is also larger than in hole doped ones. Experimental and theoretical results discussed here clearly confirm the AFM scenario of the formation of the pseudogap in both hole-doped [143, 144], and electron doped cuprates [145, 146, 147].

\section{Conclusion}

In this review we discussed DMFT $+\Sigma$ generalization of the standard dynamical meanfield theory (DMFT), which allows to include non-local correlations or additional (relative to Hubbard one) interactions (in principle of any type), while remaining within the single-impurity picture of DMFT and retaining the same set of self-consistent DMFT equations. The basic approximation of this method is the neglect of interference contributions of DMFT diagrams and additional interactions included into the analysis. Precisely this (strictly speaking not completely controllable) approximation allows to preserve the overall structure of DMFT equations, which permits to solve DMFT $+\Sigma$ equations with well developed methods used in the standard DMFT. It must be emphasized that the 
self-consistent account of additional interactions at every step of DMFT loop leads to a rather complicated procedure, equivalent to the summation of infinite classes of diagrams.

The proposed approach proved to be versatile enough to be applied to a number of problems in systems with strong electron correlations - from semi-phenomenological account of non-local short range order pseudogap fluctuations to the self-consistent scheme for metal-insulator transition in the disordered Hubbard-Anderson model and account of the effects of electron-phonon interaction in electronic spectra of strongly correlated systems. A remarkable feature of DMFT $+\Sigma$ approach is the possibility to study, along with one-particle characteristics, also the two-particle properties, i.e., in principle, any kind of response functions (optical conductivity, magnetic susceptibility, charge screening, etc.). The universality of the method allows one to hope for its successful application in a number of future problems.

Discussing all problems under consideration, one should keep in mind that in many respects similar physical results can be obtained with more sophisticated approaches, using these or other methods of direct numerical simulation. For example, similar results for the formation of the pseudogap in the single-particle characteristics of the two-dimensional Hubbard model were obtained in the cluster generalizations of DMFT [21, 22. However, these methods have specific limitations (e.g. of cluster size) and are still not widely used to calculate the two-particle properties, such as the general response functions, in particular, the optical conductivity. DMFT $+\Sigma$ approach has obvious advantages, associated with savings of computational resources. It requires a significantly lower cost of computational time, and its advantage in calculating the two-particle response functions is quite obvious. This opens up additional opportunities for the systematic comparison of various types of non-local fluctuations or additional interactions and their influence on the electronic properties of strongly correlated systems, providing intuitively clear path to the analysis of experiments and theoretical results obtained via more complicated schemes.

Rather simple generalization of our computational scheme enabled us to formulate also the generalized LDA $+\mathrm{DMFT}+\Sigma$ approach, that allows to perform calculations of all of the effects discussed above for real compounds of transition elements with strong electronic correlations. One can expect that these calculations will be useful in analyzing and explaining the new experimental data.

\section{Acknowledgements}

The authors thank Th. Pruschke for his significant contribution to the development of $\mathrm{DMFT}+\Sigma$ approach in its initial stages, as well as for providing us with very efficient NRG code that was used in most calculations.

We are also grateful to S. Borisenko and other members of Dresden ARPES group with whom ARPES studies of PCCO were performed.

Investigations of $\mathrm{Sr}(\mathrm{Ca}) \mathrm{VO}_{3}$, LSCO and $\mathrm{NCCO}$ were done in the framework of joint project together with University of Osaka (group of Prof. S. Suga and Prof. A. Sekiyama).

This work was partially supported by RFBR grant 11-02-00147 and performed within the framework of programs of Presidium of RAS "Quantum physics of condensed matter" (UB RAS 09-П-2-1009) and Physics Division of RAS "Strongly correlated electrons in solids" (UB RAS 09-T-2-1011 .) 


\section{References}

[1] Mott N F Metal-Insulator Transitions, 2nd edn. (London:Taylor and Francis, 1990)

[2] Anisimov V I, Izyumov Yu A, Electronic Structure of Strongly Correlated Materials (Berlin - Heidelberg: Springer, 2010 )

[3] Vollhardt D in Correlated Electron Systems (Ed V J Emery) (Singapore: World Scientific, 1993) p. 57

[4] Pruschke Th, Jarrell M, Freericks J K Adv. in Phys. 44210 (1995)

[5] Georges A et al. Rev. Mod. Phys. 6813 (1996)

[6] Vollhardt D AIP Conference Proceedings 1297339 (American Institute of Physics, Melville, New York, 2010)

[7] Kotliar G and Vollhardt D Physics Today 5753 (2004)

[8] Hubbard J J Proc. Roy. Soc. London Ser. A 276238 (1963); J Proc. Roy. Soc. London Ser. A 277237 (1964); J Proc. Roy. Soc. London Ser. A 281401 (1964); J Proc. Roy. Soc. London Ser. A 285542 (1965); J Proc. Roy. Soc. London Ser. A 296829 (1967); J Proc. Roy. Soc. London Ser. A 296100 (1967)

[9] Metzner W and Vollhardt D Phys. Rev. Lett. 62324 (1989)

[10] Anderson P W Phys. Rev. 12441 (1961)

[11] Pruschke Th, Grewe N Z. Phys. B 74439 (1989)

[12] Pruschke Th, Cox D L, Jarrell M Phys. Rev. B, 473553 (1993)

[13] Hirsch J E, Fye R M Phys. Rev. Lett. 562521 (1986); Jarrell M Phys. Rev. Lett. 69168 (1992); Rozenberg M, Zhang X Y, Kotliar G Phys. Rev. Lett. 69, 1236 (1992); Georges A, Krauth W, Phys. Rev. Lett. 691240 (1992); Jarrell M in Numerical Methods for lattice Quantum Many-Body Problems, (edited by D Scalapino) (Addison Wesley, 1997)

[14] K.G. Wilson, Rev. Mod. Phys. 47773840 (1975)

[15] Bulla R, Costi T A, Pruschke Th Rev. Mod. Phys. 80395 (2008)

[16] Anisimov V I et al. J. Phys. Cond. Matter 97359 (1997)

[17] Lichtenstein A I, Katsnelson M I Phys. Rev. B 57, 6884 (1998)

[18] Nekrasov I A et al. Euro. Phys. J. B 1855 (2000)

[19] Held K et al. Psi-k Newsletter 5665 (2003) 
[20] Held K et al. Int. J. Mod. Phys. B 152611 (2001); Held K et al. in Quantum Simulations of Complex Many-Body Systems: From Theory to Algorithms, (Eds J Grotendorst, D Marks, A Muramatsu) NIC Series Volume 10 (2002) p. 175; Lichtenstein A I, Katsnelson M I, Kotliar G, in Electron Correlations and Materials Properties 2nd ed. (Eds A Gonis, N Kioussis, M. Ciftan) (Kluwer Academic/Plenum, 2002) p. 428

[21] Maier Th et al. Rev. Mod. Phys. 771027 (2005)

[22] Kotliar G et al. Phys. Rev. Lett. 87186404 (2001); with periodic boundary conditions (PCDMFT), cf. Capone M Phys. Rev. B 69195105 (2004)

[23] Sadovskii M V et al. Phys. Rev. B 72155105 (2005)

[24] Kuchinskii E Z, Nekrasov I A, Sadovskii M V Pis'ma Zh. Eksp. Teor. Fiz. 82217 (2005) [JETP Lett. 82198 (2005)]

[25] Kuchinskii E Z, Nekrasov I A, Sadovskii M V Fizika Nizkikh Temperatur 32528 (2006) [Low Temp. Phys. 32398 (2006)]

[26] Kuchinskii E Z, Nekrasov I A, Sadovskii M V Phys. Rev. B 75115102 (2007)

[27] Kuchinskii E Z, Nekrasov I A, Sadovskii M V Zh. Eksp. Teor. Fiz. 133670 (2008) [JETP 106581 (2008)]

[28] Vollhardt D, Wölfle P Phys. Rev. B 224666 (1980); Phys. Rev. Lett. 48699 (1982)

[29] Sadovskii M V Diagrammatics (Singapore: World Scientific, 2006)

[30] Kotliar G et al. Phys. Rev. Lett. 87186401 (2001); Biroli G, Kotliar G Phys. Rev. B 65155112 (2002)

[31] Lichtenstein A I, Katsnelson M I, Phys. Rev. B 629283 (2000)

[32] Hettler M H, Tahvildar-Zadeh A N, Jarrel M, Phys. Rev. B 587475 (1998); Hettler M H et al. Phys. Rev. B 6112739 (2000)

[33] Maier Th et al. Phys. Rev. Lett. 851524 (2000); Eur. Phys. J. B 13613 (2000); Huscroft C et al. Phys. Rev. Lett. 86139 (2001)

[34] Kusunose H J. Phys. Soc. Japan 75054713 (2006)

[35] Toshi A, Katanin A A, Held K Phys. Rev. B 75045118 (2007)

[36] Rubtsov A N, Katsnelson M I, Lichtenstein A I Phys. Rev. B 77, 033101 (2008)

[37] Rubtsov A N, Katsnelson M I, Lichtenstein A I, Georges A Phys. Rev. B 79, 045133 (2009)

[38] Timusk T, Statt B Rep. Progr. Phys. 6261 (1999)

[39] Sadovskii M V Usp. Fiz. Nauk 171539 (2001) [Sadovskii M V Phys. Usp. 44515 (2001)] 
[40] Pines D ArXiv: cond-mat/0404151

[41] Schmalian J, Pines D, Stojkovic B Phys. Rev. Lett. 803839 (1998); Phys. Rev. B B60 667 (1999)

[42] Kuchinskii E Z, Sadovskii M V Zh. Eksp. Teor. Fiz. 1151765 (1999) [Kuchinskii E Z, Sadovskii M V JETP 88347 (1999)]

[43] Sadovskii M V Zh. Eksp. Teor. Fiz. 772070 (1979) [Sadovskii M V Sov.Phys.-JETP 50989 (1979)]

[44] Sadovskii M V Zh. Eksp. Teor. Fiz. 661720 (1974) [Sov. Phys.-JETP 39845 (1974)]

[45] Sadovskii M V, Timofeev A A J. Moscow Phys. Soc. 1391 (1991)

[46] Sadovskii M V, Strigina N A 122610 (2002) [JETP 95526 (2002)]

[47] Vilk Y M, Tremblay A-M S J. Phys. I France 71309 (1997)

[48] Ding H et al. Nature 38251 (1996)

[49] Kuchinskii E Z, Sadovskii M V Zh. Eksp. Teor. Fiz. 130477 (2006) [JETP 103415 (2006)]

[50] Armitage N P et al. Phys. Rev. Lett. 87147003 (2001)

[51] Basov D N, Timusk T Rev. Mod. Phys. 77721 (2005)

[52] Hwang J, Timusk T, Gu G D J. Phys. Cond. Matter 19125208 (2007)

[53] Lee P A,Ramakrishnan T V Rev. Mod. Phys. 57287 (1985); Belitz D, Kirkpatrick T R Rev. Mod. Phys. 66261 (1994)

[54] Mott N F Proc. Phys. Soc. A 62416 (1949)

[55] Anderson P W Phys. Rev. 1091492 (1958)

[56] Finkelshtein A F Zh. Eksp. Teor. Fiz. 84168 (1983) [Sov. Phys. JEPT 5797 (1983)]; Castellani C et al. P Phys. Rev. B 30527 (1984)

[57] Dobrosavljević V and Kotliar G Phys. Rev. Lett. 783943 (1997)

[58] Dobrosavljević V, Pastor A A, Nikolić B K, Europhys. Lett. 6276 (2003)

[59] Byczuk K, Hofstetter W, Vollhardt D, Phys. Rev. Lett. 94056404 (2005)

[60] Henseler P, Kroha J, Shapiro B Phys. Rev. B 77075101 (2008)

[61] Henseler P, Kroha J, Shapiro B Phys. Rev. B 78235116 (2008)

[62] Pezzoli M E, Becca F Phys. Rev. B 81075106 (2010)

[63] Ulmke M, Janiš V and Vollhardt D. Phys. Rev. B 5110411 (1995)

[64] Vlaming R and Vollhardt D Phys. Rev. B 454637 (1992) 
[65] Wölfle P and Vollhardt D in Anderson Localization, (Eds Y. Nagaoka and H. Fukuyama) Springer Series in Solis State Sciences, (Berlin Springer 1982) 39 p. 26

[66] Sadovskii M V in Soviet Scientific Reviews - Physics Reviews (Ed I.M. Khalatnikov) (NY:Harwood Academic Publ., 1986) 7 p. 1

[67] Wölfle $\mathrm{P}$ and Vollhardt D in Electronic Phase Transitions, (Eds W. Hanke, Yu.V. Kopaev) (Amsterdam:North-Holland, 1992) 32 p. 1

[68] Kuchinski E Z, Sadovskii M V, Suvorov V G, Erkabaev M A Zh. Eksp. Teor. Fiz. 1072027 (1995) [JETP 801122 (1995)]; Kuchinskii E Z, Erkabaev M A Fiz. Tverd. Tela 39412 (1997)

[69] Kuchinskii E Z, Kuleeva N A, Nekrasov I A, Sadovskii M V Zh. Eksp. Teor. Fiz. 137368 (2010) [JETP 110325 (2010)]

[70] Bulla R Phys. Rev. Lett. 83136 (1999); Bulla R, Costi T A, Vollhardt D Phys. Rev. B 64045103 (2001)

[71] N. Blümer. Mott-Hubbard Metal-Insulator Transition and Optical Conductivity Thesis, München 2002.

[72] Erkabaev M A, Sadovskii M V. J. Moscow Phys. Soc. 2233 (1992)

[73] Abrahams E et al. Phys. Rev. Lett. 42673 (1979)

[74] Altshuler B L, Aronov A G, Lee P A Phys. Rev. Lett. 441288 (1980)

[75] Dolan G J, Osheroff D D Phys. Rev. Lett. 43721 (1979); Bishop D J, Tsui D C, Dynes R C Phys. Rev. Lett. 441153 (1980); Uren M J, Davies R A, Pepper M J. Phys. C 13 L985 (1980)

[76] Kravchenko S V, Sarachik M P, Rep. Prog. Phys. 671 (2004)

[77] Abrahams E, Kravchenko S V, Sarachik M P Rev. Mod. Phys. 73251 (2001)

[78] Valla T et al. Phys. Rev. Lett. 832085 (1999)

[79] M Hengsberger et al. Phys. Rev. Lett. 83592 (1999)

[80] Lanzara A et al Nature 412510 (2001)

[81] Shen Z X et al. Philos. Mag. B 821349 (2002)

[82] Rotenberg E, Schaefer, Kevan S D Phys. Rev. Lett. 842925 (2000)

[83] Higashiguchi M et al. J. Electron Spectrosc. Relat. Phenom. 144-147 639 (2005)

[84] Sun Z et al. Phys. Rev. Lett. 97056401 (2006)

[85] He H et al. Phys. Rev. Lett. 861610 (2001)

[86] Hwang J Timusk T, Gu G D, Nature 427714 (2004) 
[87] Ronning F et al. Phys. Rev. B 67165101 (2003)

[88] Yoshida T et al. Phys. Rev. Lett. 95146404 (2005)

[89] Graf J et al. Phys. Rev. Lett. 98067004 (2007)

[90] Byczuk K et al. Nature Phys. 3168 (2007)

[91] Abrikosov A A, Gorkov L P, Dzyaloshinski I E Methods of Quantum Field Theory in Statistical Physics (NY:Dover Publications 1975)

[92] Nekrasov I A et al. Phys. Rev. B 73155112 (2006)

[93] Toshi A, Capone M, Castellani C, Held K. Phys. Rev. Lett. 102076402 (2009)

[94] Bulla R, Pruschke Th, Hewson A C Physica B 259-261 721 (1999)

[95] Holstein T Ann. Phys. (N.Y.) 8325 (1959)

[96] Hewson A C, Mayer D J. Phys.: Condens. Matter 175413 (2002)

[97] Koller W, Hewson A C, Edwards D M. Phys. Rev. Lett. 95256401 (2006)

[98] Hague J P J. Phys.: Condens. Matter 152535 (2003)

[99] Migdal A D Zh. Eksp. Teor. Fiz. 341438 (1958) [Sov. Phys. JETP 7999 (1958)]

[100] Kuchinskii E Z, Nekrasov I A, Sadovskii M V Phys. Rev. B 80115124 (2009)

[101] Sadovskii M V, Kuchinskii E Z, Nekrasov I A. J. Phys. Chem. Solids 72366 (2011)

[102] Koller W, Mayer D, Hewson A C Phys. Rev. B 70, 155103 (2004)

[103] Jeon G S et al. Phys. Rev. B 70125114 (2004)

[104] Koller W et al. Europhys. Lett. 66559 (2004)

[105] Born M, Oppenheimer R Ann. Phys. (Leipzig) 84457 (1927)

[106] Hohenberg P, Kohn W Phys. Rev. B 136864 (1964)

[107] Jones R O, Gunnarsson O Rev. Mod. Phys. 61689 (1989)

[108] Kohn W, Sham L J, Phys. Rev. 140 A1133 (1965); Sham L J, Kohn W Phys. Rev. 145561 (1966)

[109] Janak J F Phys. Rev. B 187165 (1978)

[110] D. M. Ceperley and B. J. Alder Phys. Rev. Lett. 45566 (1980).

[111] Andersen O K Phys. Rev. B 123060 (1975); Andersen O K, Jepsen O Phys. Rev. Lett. 532571 (1984)

[112] Müller-Hartmann E Z. Phys. B 74507 (1989); 76211 (1989) 
[113] Wahle J et al. Phys. Rev. B 5812749 (1998)

[114] Held K Adv. Phys. 56829 (2007)

[115] Karolak M et al. Journal of Electron Spectroscopy and Related Phenomena 18111 (2010)

[116] Zölfl M B et al. Phys. Rev. B. 6112810 (2000)

[117] Gunnarsson O et al. Phys. Rev. B 39, 1708 (1989)

[118] Aryasetiawan F, Imada M, Georges A, Kotliar G, Biermann S, Lichtenstein A I Phys. Rev. B 70195104 (2004)

[119] Miyake T, Aryasetiawan F Phys. Rev. B 77085122 (2008)

[120] Lambin Ph Vigneron J P Phys. Rev. B 293430 (1984)

[121] Andersen O K, Saha-Dasgupta T Phys. Rev. B 62 R16219 (2000); Andersen O K et al. Psi-k Newsletter 4586 (2001); Andersen O K, Saha-Dasgupta T, Ezhov S Bull. Mater. Sci. 2619 (2003)

[122] Anisimov V I Phys. Rev. B 71125119 (2005)

[123] Fujimori A et al. Phys. Rev. Lett. 691796 (1992)

[124] Aiura A et al. Phys. Rev. B. 476732 (1993)

[125] Inoue I-H et al. Phys. Rev. Lett. 742539 (1995)

[126] Inoue I-H et al. Phys. Rev. B. 584372 (1998)

[127] Morikawa K et al. Phys. Rev. B. 5213711 (1995)

[128] Maiti K et al. Europhys. Lett. 55246 (2001)

[129] Sekiyama A et al. Phys. Rev. Lett. 93156402 (2004)

[130] Inoue I-H et al. Physica C. 235-240 1007 (1994)

[131] Rozenberg M J et al. Phys. Rev. Lett. 764781 (1996)

[132] Solovyev I et al. Phys. Rev. B. 537158 (1996)

[133] Makino H et al. Phys. Rev. B. 584384 (1998)

[134] Jarrell M, Gubernatis J E Physics Reports 269133 (1996)

[135] Krause M O, Oliver J H J. Phys. Chem. Ref. Data. 8329 (1979)

[136] Inoue I-H et al. Phys. Rev. Lett. 88236403 (2002)

[137] Pavarini E et al. Phys. Rev. Lett. 92176403 (2004)

[138] Pavarini E et al.New J. Phys. 7188 (2005) 
[139] Yoshida T et al. Phys. Rev. Lett. 95146404 (2005)

[140] Sadovskii M V In Strings, Branes, Lattices, Networks, Pseudogaps and Dust (Moscow: Scientific World, 2007) p. 357 (in Russian) [English translation arXiv: cond-mat/0408489]

[141] Damascelli A, Hussain Z, Shen Z-X Rev. Mod. Phys. 75473 (2003)

[142] Campuzano J C Norman M R, Randeria M in Physics of Superconductors (Eds K. H. Bennemann, J. B. Ketterson (Berlin: Springer, 2004), Vol II, p. 167-273; J. Fink et al. in Lecture Notes in Physics, (Berlin: Springer, 2007) 715 p. 295; Zhou et al. in Handbook of High-Temperature Superconductivity: Theory and Experiment (Ed J. R. Schrieffer) (Berlin:Springer, 2007), p. 87

[143] Kuchinskii E Z et al. Zh. Eksp. Teor. Fiz. 131908 (2007) [JETP 104792 (2007)]

[144] Nekrasov I A et al. Zh. Eksp. Teor. Fiz. 1371133 (2010) [JETP 110989 (2007)]

[145] Nekrasov I A et al. J. Phys. Chem. Solids 693269 (2008)

[146] Kokorina E E et al. Zh. Eksp. Teor. Fiz. 134968 (2008) [JETP 107828 (2008)]

[147] Nekrasov I A et al. Phys. Rev. B 80140510 (2009)

[148] Nekrasov I A, Kuchinskii E Z, Sadovskii M V J. Phys. Chem. Solids 72371 (2011)

[149] Hedin L and Lundqvist B I J. Phys. C 42064 (1971); von Barth U, Hedin L J. Phys. C 1629 (1972)

[150] Andersen O K et al. J. Phys. Chem. Solids 561573 (1995)

[151] Zobkalo I A et al. Solid State Comm. 80921 (1991) Motoyama E M et al. Nature 445186 (2007)

[152] M. Hücker et al. Phys. Rev. B 71094510 (2005)

[153] Armitage N P et al. Phys. Rev. Lett. 88257001 (2002)

[154] Kaminski A et al. Phys. Rev. Lett. 88257001 (2002)

[155] Borisenko S V Phys. Rev. Lett. 844453 (2000)

[156] Massidda S et al. Physica C 157571 (1989); Matsuno S, Kanimura H J. of Superconductivity 7517 (1994)

[157] Onose Y et al. Phys. Rev. Lett. 87217001 (2001)

[158] Quijada M A et al. Phys. Rev. B 6014917 (1999) 